\title{
Second order transport coefficient from the chiral anomaly at weak coupling: Diagrammatic resummation
}

\author{
Amadeo Jimenez-Alba ${ }^{1, *}$ and Ho-Ung Yee ${ }^{2,3, \dagger}$ \\ ${ }^{1}$ Instituto de Fisica Teorica IFT-UAM/CSIC, Universidad Autonoma de Madrid, \\ 28049 Cantoblanco, Spain \\ ${ }^{2}$ Department of Physics, University of Illinois, Chicago, Illinois 60607, USA \\ ${ }^{3}$ Brookhaven National Laboratory, RIKEN-BNL Research Center, Upton, New York 11973-5000, USA
}

(Received 9 June 2015; published 22 July 2015)

\begin{abstract}
We compute one of the second order transport coefficients arising from the chiral anomaly in a hightemperature weakly coupled regime of quark-gluon plasma. This transport coefficient is responsible for the $C P$-odd current that is proportional to the time derivative of the magnetic field, and can be considered as a first correction to the chiral magnetic conductivity at finite, small frequency. We observe that this transport coefficient has a nonanalytic dependence on the coupling as $\sim 1 /\left(g^{4} \log (1 / g)\right)$ at the weak coupling regime, which necessitates a resummation of infinite ladder diagrams with leading pinch singularities to get a correct leading log result, a feature quite similar to what one finds in the computation of electric conductivity. We formulate and solve the relevant $C P$-odd Schwinger-Dyson equation in real-time perturbation theory that reduces to a coupled set of second order differential equations at leading log order. Our result for this second order transport coefficient indicates that chiral magnetic current has some resistance to the time change of the magnetic field; this shall be called the "chiral induction effect." We also discuss the case of color current induced by a color magnetic field.
\end{abstract}

DOI: 10.1103/PhysRevD.92.014023

PACS numbers: 12.38.Mh, 11.10.Wx, 12.38.Aw

\section{INTRODUCTION}

The chiral anomaly is an intriguing quantum mechanical phenomenon arising from an interplay between charge and chirality of massless particles such as chiral fermions. It has recently been appreciated that the chiral anomaly may induce interesting parity-odd transport phenomena in the plasmas of such particles [1-5]; at the lowest order in derivative expansion of hydrodynamics (that is, at the first order) it has been shown that the second law of thermodynamics dictates the existence of such phenomena [6], including the chiral magnetic effect [3] and the chiral vortical effect $[7,8]$. Moreover, the magnitudes of these transport phenomena in the static, homogeneous limit are fixed by underlying anomaly coefficients and are not renormalized by interactions. This universality has been confirmed explicitly in both weak [9-12] and strong [13-17] coupling computations, and has been proven in hydrostatic constraints analysis [18-20]. There is also evidence in favor of them in lattice simulations [21-25]. Recent results from heavy-ion experiments at the RHIC [26-30] and the LHC [31] seem consistent with the predictions from chiral magnetic and vortical effects [32,33] (as well as chiral magnetic wave [34-38]), and, quite interestingly, there has been a successful experimental test of the chiral magnetic effect in Dirac/Weyl semimetals that feature chiral fermionic excitations [39] (the spin

\footnotetext{
*amadeo.j@gmail.com

†hyee@uic.edu
}

degree of freedom in this case arises from an internal degeneracy; in other words, it is a pseudospin). Therefore, the existence and the magnitudes of these transport phenomena at lowest order (i.e., first order) in derivative expansion seem by now quite robust. One can also generalize them to all even space-time dimensions higher than four [40-46].

As we go beyond the lowest order in derivatives, the possible anomaly-induced transport phenomena become numerous: in four dimensions there are 13 possible second order anomalous transport coefficients in the current and energy-momentum tensor in a conformal plasma [40] (and more in nonconformal plasma [47]), while the second law of thermodynamics seems to constrain only eight combinations of them [40]. Some of these constraints have been confirmed in a strong coupling computation [48]. Interestingly, the values of these anomalous second order transport coefficients, although they are proportional to anomaly coefficients, do depend on the dynamics of the microscopic theory up to the mentioned constraints; therefore, computing them in weak and strong coupling regimes is a nontrivial, but worthwhile, task in any theoretical model.

The purpose of this work is to take a small step in computing these second order anomalous transport coefficients in weakly coupled gauge theories, with QCD and electroweak theory in mind. Our current study will be based on diagrammatic techniques, and we hope to address a similar computation in a chiral kinetic theory framework [49-53] in a separate work. We will show that one 
particular second order anomalous transport coefficient in the charge current has a nonanalytic dependence on the coupling constant, $\sim 1 / g^{4} \log (1 / g)$, which is similar to what one finds in the shear viscosity and electric conductivity $[54,55]$ (and in the chiral electric separation conductivity [56] as well). ${ }^{1}$ This transport coefficient appears in the second derivative correction to the current constitutive relation as

$$
\nu_{(2)}^{\mu} \sim \xi_{5} \epsilon^{\mu \nu \alpha \beta} u_{\nu} \mathcal{D}_{\alpha} E_{\beta},
$$

where $E_{\mu}=F_{\mu \nu} u^{\nu}$ is the electric field strength in a local fluid rest frame defined by $u^{\mu}$, and we followed the notation introduced in Ref. [40] to denote the transport coefficient $\xi_{5}$. Using the Bianchi identity, one can replace $\epsilon^{\mu \nu \alpha \beta} u_{\nu} \mathcal{D}_{\alpha} E_{\beta}$ with $u^{\nu} \mathcal{D}_{\nu} B^{\mu}$, where $B^{\mu}=\frac{1}{2} \epsilon^{\mu \nu \alpha \beta} u_{\nu} F_{\alpha \beta}$ is the magnetic field strength in the local rest frame; this means that $\xi_{5}$ can be viewed as a first correction to the static chiral magnetic effect at finite frequency. More explicitly, it appears in the anomalous part of the current density as

$$
\vec{J}=\cdots+\sigma_{\chi} \vec{B}+\xi_{5} \frac{d \vec{B}}{d t}+\cdots,
$$

where $\sigma_{\chi}$ is the topologically protected value of chiral magnetic conductivity at zero frequency. $\xi_{5}$ is parity $(\mathrm{P})$ and charge conjugation-parity $(C P)$ odd, so it must arise from the chiral anomaly.

As was observed first by Jeon [57], in diagrammatic language, the nonanalytic behavior in the coupling dependence is signaled by the presence of pinch singularities in multiloop ladder diagrams of two-point correlation functions; this necessitates a resummation of all ladder graphs, by solving a Schwinger-Dyson-type equation, to get a leading log result. It was previously observed in Ref. [12] that the zero frequency-momentum limit of the P-odd part of the one-loop correlation function does not have pinch singularity, reproducing the correct static value of chiral magnetic conductivity. We first motivate our study by observing an appearance of pinch singularity in the P-odd part of a one-loop diagram at first order in frequency, which enters in the Kubo formula for $\xi_{5}$. Following intuitions from the computation of electric conductivity [58-60], we then identify multiloop ladder graphs whose Podd parts contain a chain of pinch singularities that have to be resummed to get a correct leading $\log$ result for $\xi_{5}$. The emerging Schwinger-Dyson equation is more difficult to solve than that for the electric conductivity, because we

\footnotetext{
${ }^{1}$ Our definition of transport coefficients does not include a trivial $e^{2}$ factor from the definition of electromagnetic current, which is $e$ times the fermion number current. Therefore, all quantities in our work are defined with the fermion number current. For example, the electric conductivity will be $\sim 1 /\left(e^{4} \log (1 / e)\right)$ and the chiral magnetic conductivity at zero frequency for a single right-handed Weyl fermion is $\sigma_{\chi}=\frac{\mu}{4 \pi^{2}}$.
}

need to keep finite external momentum $\vec{k}$ (up to first order in $\vec{k}$ ) to extract the P-odd part of the correlation function. In Sec. IV, we prove an important fact that all $\vec{k}$ dependence in the denominators of pinching propagators do not contribute, up to first order in $\vec{k}$, to the P-odd part that we are interested in, allowing us to neglect them in the denominators of pinching propagators. The necessary $\vec{k}$ dependence for P-odd correlation functions arises only from the spinor projection part of the fermion propagators. With this important simplification, we are able to reduce the leading log part of the P-odd Schwinger-Dyson equation into a coupled set of second order differential equations, which can be solved numerically. Along the way, we develop and use the sum rule for the P-odd part of hard thermal loop (HTL) photon spectral density, which is summarized in Appendix A.

For most of our presentation, we will consider a single species of Weyl fermion in quantum electrodynamics (QED) for simplicity; a generalization to a finite number of species of Weyl and Dirac fermions, as well as to a nonAbelian $S U\left(N_{c}\right)$ gauge theory, is trivial at our leading log order. We will describe this generalization in our discussion section at the end. Our results are summarized as follows: For QED with a single right-handed Weyl fermion, we have

$$
\xi_{5}=\frac{-3.006}{e^{4} \log (1 / e)} \frac{\mu}{T}
$$

For two-flavor massless QCD $\left(N_{c}=3\right)$ with $Q_{u}=3 / 2$ and $Q_{d}=-1 / 3$, our result is

$$
\xi_{5}^{\mathrm{QCD}}=\left(Q_{u}^{2}+Q_{d}^{2}\right) \frac{-3.6}{g^{4} \log (1 / g)} \frac{\mu_{A}}{T}=\frac{-2.003}{g^{4} \log (1 / g)} \frac{\mu_{A}}{T},
$$

where $\mu_{A}$ is an axial chemical potential. The sign of $\xi_{5}$ compared to the zero-frequency value $\sigma_{\chi}\left(=\frac{\mu}{4 \pi^{2}}\right.$ for QED) is a meaningful dynamical result. A relative negative sign between the two means that the chiral magnetic current has some resistance to the change of the magnetic field. We shall call this the "chiral induction effect."

\section{PINCH SINGULARITY IN THE P-ODD PART AT ONE LOOP}

In this section, let us motivate our work by observing an appearance of pinch singularity in the P-odd part of a oneloop diagram at first order in frequency $\omega$. It will also serve to fix our notations and conventions. For simplicity, we will consider the case of a single Weyl fermion species of unit charge in QED plasma at finite equilibrium temperature $T$, as the generalization to multiflavors or non-Abelian gauge groups is simple (we will summarize it at the end of the paper). Throughout our analysis, we will use the real-time Schwinger-Keldysh formalism in " $r a$ " basis to compute the retarded current-current correlation function that contains the chiral magnetic conductivity $\sigma_{\chi}(k)$ in its P-odd part, 

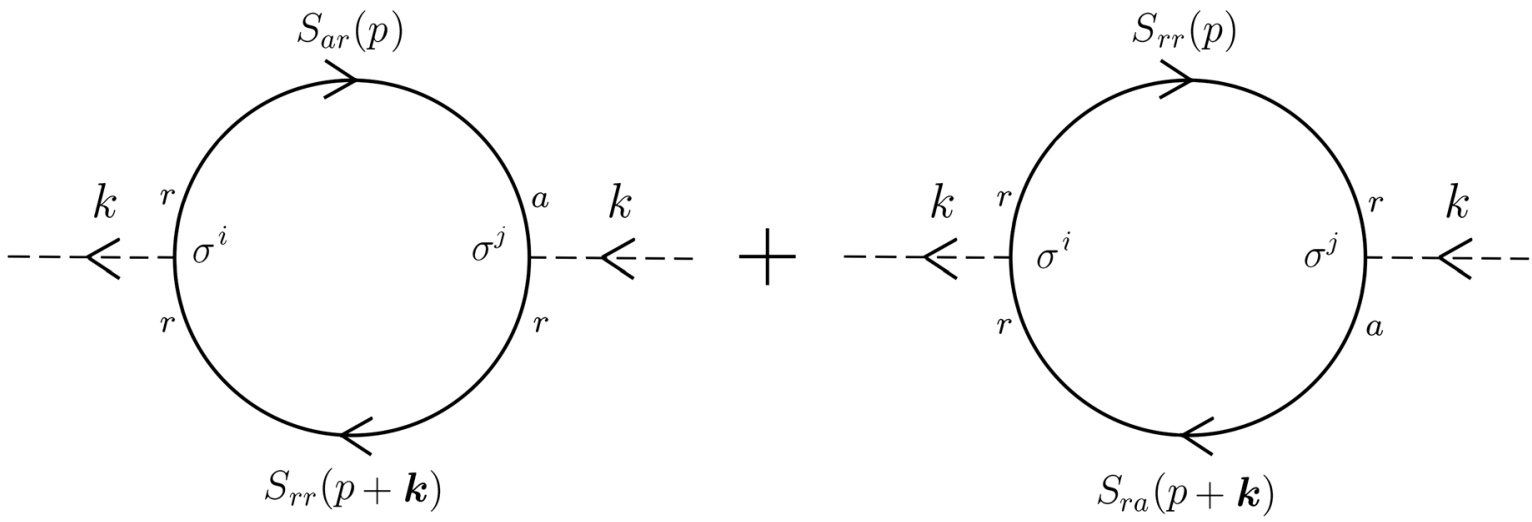

FIG. 1. Diagrams responsible for the retarded response at one loop in the $r a$ basis.

$$
\left\langle J^{i}(k) J^{j}(-k)\right\rangle_{R}^{\mathrm{P}-\text { odd }}=i \sigma_{\chi}(k) \epsilon^{i j l} k^{l},
$$

where italic letters run over the three spatial dimensions and $k=\left(k^{0}, \boldsymbol{k}\right) \equiv(\omega, \boldsymbol{k})$ is an external four-momentum. Note that in $r a$ basis, the retarded two-point function is equal to

$$
\left\langle J^{i}(k) J^{j}(-k)\right\rangle_{R}=(-i)\left\langle J_{r}^{i}(k) J_{a}^{j}(-k)\right\rangle_{\mathrm{SK}} \equiv(-i) G_{(r a)}^{i j}(k),
$$

where the subscript SK in the second term emphasizes that it is computed in the Schwinger-Keldysh path integral with $J_{r} \equiv 1 / 2\left(J_{1}+J_{2}\right)$ and $J_{a} \equiv J_{1}-J_{2}$ (1 and 2 denote the two time contours in the Schwinger-Keldysh formalism). We follow the notations in Ref. [12] for consistency. ${ }^{2}$ Explicitly,

$$
J_{r}^{\mu}=\psi_{r}^{\dagger} \sigma^{\mu} \psi_{r}+\frac{1}{4} \psi_{a}^{\dagger} \sigma^{\mu} \psi_{a}, \quad J_{a}^{\mu}=\psi_{r}^{\dagger} \sigma^{\mu} \psi_{a}+\psi_{a}^{\dagger} \sigma^{\mu} \psi_{r},
$$

with $\sigma^{\mu}=\left(\mathbf{1}_{2 \times 2}, \vec{\sigma}\right)$ in terms of a two-component Weyl spinor field $\psi$. Therefore, the task is to compute the P-odd part (or antisymmetric part in $i, j$ indices) of the $(r a)$ correlator $G_{(r a)}^{i j}(k)$ for small frequency momentum.

The zero frequency-momentum limit of $\sigma_{\chi}(k)$ has been shown to be universally

$$
\lim _{k \rightarrow 0} \lim _{\omega \rightarrow 0} \sigma_{\chi}(k)=\frac{\mu}{4 \pi^{2}},
$$

and, in particular, no pinch singularity appears in this limit, as shown in Ref. [12]. Our $\xi_{5}$ appears in first order expansion in $\omega=k^{0}$ (while still taking the zero momentum limit $\boldsymbol{k} \rightarrow 0$ ),

\footnotetext{
${ }^{2}$ In literature, the retarded function is often chosen to be denoted by $G_{(r a)}$, which we find confusing.
}

$$
\lim _{k \rightarrow 0} \sigma_{\chi}(k)=\frac{\mu}{4 \pi^{2}}-i \xi_{5} \omega+\mathcal{O}\left(\omega^{2}\right)
$$

However, since the P-odd part of $G_{(r a)}^{i j}$ contains a linear term in $\boldsymbol{k}$ in defining $\sigma_{\chi}(k)$,

$$
G_{(r a)}^{i j, \mathrm{P} \text {-odd }}(k)=-\sigma_{\chi}(k) \epsilon^{i j l} k^{l},
$$

we have to keep the $\boldsymbol{k}$ dependence in $G_{(r a)}^{i j}(k)$ up to first order in $\boldsymbol{k}$, and then take the $\boldsymbol{k} \rightarrow 0$ limit of the P-odd coefficient $\sigma_{\chi}(k)$. This essentially means that we need to keep finite $\boldsymbol{k}$ in the Schwinger-Dyson equation for ladder resummation; this is in contrast to the case of electric conductivity, where one can set $\boldsymbol{k}=0$ from the very outset, greatly simplifying the analysis. Despite this difficulty, we will be able to solve the Schwinger-Dyson equation for the P-odd part of $G_{(r a)}^{i j}(k)$ and extract the coefficient $\xi_{5}$. At one loop there are two Feynman diagrams for $G_{(r a)}^{i j}(k)$ in realtime formalism, as depicted in Fig. 1. The fermion propagators are given by

$$
\begin{gathered}
S_{r a}(p)=\sum_{s= \pm} \frac{i}{p^{0}-s|\boldsymbol{p}|+i \zeta / 2} \mathcal{P}_{s}(\boldsymbol{p}), \\
S_{a r}(p)=\sum_{s= \pm} \frac{i}{p^{0}-s|\boldsymbol{p}|-i \zeta / 2} \mathcal{P}_{s}(\boldsymbol{p}), \\
S_{r r}(p)=\left(\frac{1}{2}-n_{+}\left(p^{0}\right)\right) \rho(p),
\end{gathered}
$$

where $n_{ \pm}\left(p^{0}\right)=1 /\left(e^{\beta\left(p^{0} \mp \mu\right)}+1\right)$, the spectral density $\rho$ is

$$
\rho(p)=\sum_{t= \pm} \frac{\zeta}{\left(p^{0}-t|\boldsymbol{p}|\right)^{2}+(\zeta / 2)^{2}} \mathcal{P}_{t}(\boldsymbol{p}),
$$

and we introduce the damping rate $\zeta \sim g^{2} \log (1 / g) T$ in the propagators, which will be needed to regularize possible pinch singularities (this is essential in order to have a 
nonanalytic dependence on the coupling constant). It is important to observe a thermal relation

$$
S_{r r}(p)=\left(\frac{1}{2}-n_{+}\left(p^{0}\right)\right)\left(S_{r a}(p)-S_{a r}(p)\right)
$$

which plays a central role in our analysis. The projection operators $\mathcal{P}_{ \pm}(\boldsymbol{p})$ are defined as

$$
\mathcal{P}_{ \pm}(\boldsymbol{p}) \equiv \frac{1}{2}\left(\mathbf{1} \pm \frac{\boldsymbol{\sigma} \cdot \boldsymbol{p}}{|\boldsymbol{p}|}\right)=\mp \frac{\bar{\sigma} \cdot p_{ \pm}}{2|\boldsymbol{p}|},
$$

where $\bar{\sigma}^{\mu}=(\mathbf{1},-\boldsymbol{\sigma})$ and $p_{ \pm}^{\mu} \equiv( \pm|\boldsymbol{p}|, \boldsymbol{p})$. Our metric convention is $(-,+,+,+)$. The operators $\mathcal{P}_{ \pm}(\boldsymbol{p})$ project onto particle and antiparticle states, respectively, with given momentum $\boldsymbol{p}$, and the $(s, t)$ summation in the above physically represents distinctive contributions from particles and antiparticles.

The one-loop expression for $G_{(r a)}^{i j}(k)$ from the two Feynman diagrams is

$$
\begin{aligned}
& (-1) \int \frac{d^{4} p}{(2 \pi)^{4}} \operatorname{tr}\left[\sigma^{i} S_{r a}(p+k) \sigma^{j} S_{r r}(p)\right. \\
& \left.\quad+\sigma^{i} S_{r r}(p+k) \sigma^{j} S_{a r}(p)\right],
\end{aligned}
$$

where the $(-1)$ in front comes from fermion statistics. In Ref. [12] it was shown that after extracting $\epsilon^{i j l} k^{l}$ for the P-odd part, the limit $\boldsymbol{k} \rightarrow 0$ and $\omega \rightarrow 0$ commutes and produces the correct result $\mu / 4 \pi^{2}$, without featuring pinch singularity. However, we will see that the $\omega \rightarrow 0$ limit hides the pinch singularity that appears at first order in $\omega$ for $\xi_{5}$. Using the thermal relation (2.11) to replace $S_{r r}$ with $\left(S_{r a}-S_{a r}\right)$, we have several combinations of $S_{r a}$ and $S_{a r}$. From the well-known fact (see Ref. [57]) that the pinch singularity appears only from the pair of $S_{r a}$ and $S_{a r}$ sharing a same momentum, ${ }^{3}$ let us select only terms that potentially contain pinch singularity; this results in

$$
\begin{aligned}
G_{(r a)}^{i j \text { Pinch }}(k)= & \int \frac{d^{4} p}{(2 \pi)^{4}}\left(n_{+}\left(p^{0}+\omega\right)\right. \\
& \left.-n_{+}\left(p^{0}\right)\right) \operatorname{tr}\left[\sigma^{i} S_{r a}(p+k) \sigma^{j} S_{a r}(p)\right] .
\end{aligned}
$$

It is clear from this expression that the $\omega \rightarrow 0$ limit does not produce a pinch singularity, because $\left(n_{+}\left(p^{0}+\omega\right)-\right.$ $\left.n_{+}\left(p^{0}\right)\right) \approx\left(d n_{+}\left(p^{0}\right) / d p^{0}\right) \omega+\mathcal{O}\left(\omega^{2}\right)$ already gives a linear factor in $\omega$. Moreover, one can set $\omega \rightarrow 0$ in the rest expression, as we are only interested in the linear term in $\omega$ for $\xi_{5}$. In computing the above using (2.7) and (2.8) for $S_{r a}$ and $S_{a r}$, let us recall that the chosen $s= \pm$, representing a particle or an antiparticle from $S_{r a}$, must be the same $s$

\footnotetext{
${ }^{3}$ This is because $S_{r a}\left(S_{a r}\right)$ has particle poles slightly below (above) the real axis by an amount $\pm i \zeta / 2$, so that the residue of their product contains a factor of $1 / \zeta$, which is the (regularized) pinch singularity. This also means that $s= \pm$ in (2.7) and (2.8) must be common in the $S_{r a}$ and $S_{a r}$ pair, which causes a pinch singularity.
}

chosen in $S_{a r}$ in order to obtain a pinch singularity in their product. Therefore, we have

$$
\begin{aligned}
G_{(r a)}^{i j, \text { Pinch }}(k) \approx & \omega \int \frac{d^{4} p}{(2 \pi)^{4}}\left(\frac{d n_{+}\left(p^{0}\right)}{d p^{0}}\right) \\
& \times \operatorname{tr}\left[\sigma^{i} S_{r a}(p+k) \sigma^{j} S_{a r}(p)\right] \\
\approx & \omega \int \frac{d^{4} p}{(2 \pi)^{4}}\left(\frac{d n_{+}\left(p^{0}\right)}{d p^{0}}\right) \sum_{s= \pm} \\
& \times \frac{i^{2} \operatorname{tr}\left[\sigma^{i} \mathcal{P}_{s}(\boldsymbol{p}+\boldsymbol{k}) \sigma^{j} \mathcal{P}_{s}(\boldsymbol{p})\right]}{\left(p^{0}-s|\boldsymbol{p}+\boldsymbol{k}|+i \zeta / 2\right)\left(p^{0}-s|\boldsymbol{p}|-i \zeta / 2\right)} .
\end{aligned}
$$

To identify the P-odd structure containing $\epsilon^{i j l} k^{l}$ in the small $\boldsymbol{k} \rightarrow 0$ limit, we first note that there are two possible sources of $\boldsymbol{k}$ dependence: one is from the denominator and the other is from the projection operators in the numerator. Recall that we need only up to first order in $\boldsymbol{k}$ because we take the $\boldsymbol{k} \rightarrow 0$ limit after extracting the $\epsilon^{i j l} k^{l}$ piece. If one expands the denominator to linear order in $\boldsymbol{k}$, we then need to put $\boldsymbol{k}=0$ in the projection operators. The resulting trace using (2.12) gives

$\operatorname{tr}\left[\sigma^{i} \mathcal{P}_{s}(\boldsymbol{p}) \sigma^{j} \mathcal{P}_{s}(\boldsymbol{p})\right]=\frac{1}{4|\boldsymbol{p}|^{2}} \operatorname{tr}\left[\sigma^{i}\left(\bar{\sigma} \cdot p_{s}\right) \sigma^{j}\left(\bar{\sigma} \cdot p_{s}\right)\right]=\frac{p^{i} p^{j}}{|\boldsymbol{p}|^{2}}$,

where we use

$$
\operatorname{tr}\left[\sigma^{\mu} \bar{\sigma}^{\nu} \sigma^{\alpha} \bar{\sigma}^{\beta}\right]=2\left(g^{\mu \nu} g^{\alpha \beta}-g^{\mu \alpha} g^{\nu \beta}+g^{\mu \beta} g^{\nu \alpha}\right)+2 i \epsilon^{\mu \nu \alpha \beta}
$$

and $p_{s}^{2}=0$. It is clear that this contribution does not lead to a P-odd contribution that should be antisymmetric in $i$ and $j$. Hence, we can ignore $\boldsymbol{k}$ dependence in the denominator, which allows us to use the ordinary techniques dealing with pinch singularity in the $\boldsymbol{k}=0$ limit. In Sec. IV, we will prove that this simplification generalizes to all order ladder diagrams; that is, the $\boldsymbol{k}$ dependences in the denominators appearing in the ladder diagrams do not contribute to a P-odd part of the correlation function up to first order in $\boldsymbol{k}$, and, hence, can be ignored.

In computing P-odd $\boldsymbol{k}$ dependence in $\operatorname{tr}\left[\sigma^{i} \mathcal{P}_{s}(\boldsymbol{p}+\right.$ $\left.\boldsymbol{k}) \sigma^{j} \mathcal{P}_{s}(\boldsymbol{p})\right]$ using (2.12), one can replace $\mathcal{P}_{s}(\boldsymbol{p}+\boldsymbol{k})=$ $-s \bar{\sigma} \cdot(p+k)_{s} /(2|\boldsymbol{p}+\boldsymbol{k}|)$ with $-s \bar{\sigma} \cdot(p+k)_{s} /(2|\boldsymbol{p}|)$ by the same reason as above; we have

$$
\begin{aligned}
\operatorname{tr} & {\left[\sigma^{i} \mathcal{P}_{s}(\boldsymbol{p}+\boldsymbol{k}) \sigma^{j} \mathcal{P}_{s}(\boldsymbol{p})\right] } \\
& \sim \frac{1}{4|\boldsymbol{p}|^{2}} \operatorname{tr}\left[\sigma^{i}\left(\bar{\sigma} \cdot(p+k)_{s}\right) \sigma^{j}\left(\bar{\sigma} \cdot p_{s}\right)\right] \\
& \sim \frac{2 i}{4|\boldsymbol{p}|^{2}} \epsilon^{i \mu j \nu}\left((p+k)_{s}\right)_{\mu}\left(p_{s}\right)_{\nu}
\end{aligned}
$$


where in the second line, we use the fact that the P-odd contribution can come only from the last P-odd term in the $\sigma$-matrix trace (2.17). Our symbol $\sim$ cares only for the P-odd part that is linear in $k$. When $\mu=0$ and $\nu=l$, we have

$$
s \frac{i}{2|\boldsymbol{p}|^{2}} \epsilon^{i j l}|\boldsymbol{p}+\boldsymbol{k}| p^{l} \sim s \frac{i}{2|\boldsymbol{p}|^{3}} \epsilon^{i j l}(\boldsymbol{p} \cdot \boldsymbol{k}) p^{l} \sim s \frac{i}{6|\boldsymbol{p}|} \epsilon^{i j l} k^{l},
$$

where we use $|\boldsymbol{p}+\boldsymbol{k}| \approx|\boldsymbol{p}|+(\boldsymbol{p} \cdot \boldsymbol{k}) /|\boldsymbol{p}|+\mathcal{O}\left(\boldsymbol{k}^{2}\right)$, and we replace $p^{m} p^{l} \rightarrow(1 / 3) \delta^{m l}|\boldsymbol{p}|^{2}$ because the angular $\boldsymbol{p}$ integration in the final expression (2.15) is isotropic. When $\mu=l$ and $\nu=0$, we have

$$
-s \frac{i}{2|\boldsymbol{p}|^{2}} \epsilon^{i j l}\left(p^{l}+k^{l}\right)|\boldsymbol{p}| \sim-s \frac{i}{2|\boldsymbol{p}|} \epsilon^{i j l} k^{l} .
$$

Therefore, summing these two possibilities gives us the P-odd part of $\operatorname{tr}\left[\sigma^{i} \mathcal{P}_{s}(\boldsymbol{p}+\boldsymbol{k}) \sigma^{j} \mathcal{P}_{s}(\boldsymbol{p})\right]$ as

$$
\operatorname{tr}\left[\sigma^{i} \mathcal{P}_{s}(\boldsymbol{p}+\boldsymbol{k}) \sigma^{j} \mathcal{P}_{s}(\boldsymbol{p})\right] \sim-s \frac{i}{3|\boldsymbol{p}|} \epsilon^{i j l} k^{l},
$$

and from (2.15) we have

$$
\begin{aligned}
G_{(r a)}^{i j, \text { Pinch }} \sim & i \omega \epsilon^{i j l} k^{l} \int \frac{d^{4} p}{(2 \pi)^{4}}\left(\frac{d n_{+}\left(p^{0}\right)}{d p^{0}}\right) \frac{1}{3|\boldsymbol{p}|} \sum_{s= \pm} \\
& \times \frac{s}{\left(p^{0}-s|\boldsymbol{p}|+i \zeta / 2\right)\left(p^{0}-s|\boldsymbol{p}|-i \zeta / 2\right)} .
\end{aligned}
$$

The remaining computation is a standard procedure dealing with pinch singularity appearing in the denominators of (2.22). The $p^{0}$ integration can be done in the complex $p^{0}$ plane by closing the contour in either the upper or lower half-plane. The leading singularity appears from the pole $p^{0}=s|\boldsymbol{p}| \pm i \zeta / 2$, where the residue contains a factor of $1 /( \pm i \zeta)$; this gives a leading order contribution at the weak coupling limit since $\zeta \sim g^{2} \log (1 / g) T$. Once this $1 / \zeta$ term is identified from the residue of the denominators in (2.22), one can neglect $\zeta$ in the pole location $p^{0} \approx s|\boldsymbol{p}|$ for all other terms, as it engenders only higher order terms in $g$. This is because the $p$ integration has its dominant support in the region $|\boldsymbol{p}| \sim T$ while $\zeta \sim g^{2} \log (1 / g) T \ll|\boldsymbol{p}|$. Therefore, one can effectively replace the two denominators in (2.22) with

$$
\frac{1}{\left(p^{0}-s|\boldsymbol{p}|+i \zeta / 2\right)\left(p^{0}-s|\boldsymbol{p}|-i \zeta / 2\right)} \rightarrow \frac{2 \pi i}{i \zeta} \delta\left(p^{0}-s|\boldsymbol{p}|\right),
$$

which will be used frequently in the following sections. This gives us

$$
\left.G_{(r a)}^{i j \text { Pinch }} \sim i \omega \epsilon^{i j l} k^{l} \frac{1}{\zeta} \int \frac{d^{3} \boldsymbol{p}}{(2 \pi)^{3}} \frac{1}{3|\boldsymbol{p}|} \sum_{s= \pm} s\left(\frac{d n_{+}\left(p^{0}\right)}{d p^{0}}\right)\right|_{p^{0}=s \boldsymbol{p} \mid},
$$

where we ignore the momentum dependence of $\zeta$ for now, which is not strictly valid (we will be more precise in our full ladder resummation in the next section). From $d n_{+}\left(p^{0}\right) / d p^{0}=-\beta n_{+}\left(p^{0}\right)\left(1-n_{+}\left(p^{0}\right)\right)$ and $n_{+}(-|\boldsymbol{p}|)=$ $1-n_{-}(|\boldsymbol{p}|)$, the integral becomes

$$
\begin{aligned}
\left.\int \frac{d^{3} \boldsymbol{p}}{(2 \pi)^{3}} \frac{1}{3|\boldsymbol{p}|} \sum_{s= \pm} s\left(\frac{d n_{+}\left(p^{0}\right)}{d p^{0}}\right)\right|_{p^{0}=s|\boldsymbol{p}|} \\
=-\beta \int \frac{d^{3} \boldsymbol{p}}{(2 \pi)^{3}} \frac{1}{3|\boldsymbol{p}|}\left(n_{+}(|\boldsymbol{p}|)\left(1-n_{+}(|\boldsymbol{p}|)\right)\right. \\
\quad-\left(n_{-}(|\boldsymbol{p}|)\left(1-n_{-}(|\boldsymbol{p}|)\right)\right) \\
=-\frac{\beta}{6 \pi^{2}} \int_{0}^{\infty} d|\boldsymbol{p} \| \boldsymbol{p}|\left(n_{+}(|\boldsymbol{p}|)\left(1-n_{+}(|\boldsymbol{p}|)\right)\right. \\
\quad--\left(n_{-}(|\boldsymbol{p}|)\left(1-n_{-}(|\boldsymbol{p}|)\right)\right) \\
=-\frac{\mu}{6 \pi^{2}}
\end{aligned}
$$

and we finally have a one-loop expression

$$
\begin{aligned}
G_{(r a)}^{i j \text { Pinch }} & \sim-i \omega \frac{\mu}{6 \pi^{2} \zeta} \epsilon^{i j l} k^{l} \equiv i \omega \xi_{5}^{1-\text { loop }} \epsilon^{i j l} k^{l}, \\
\xi_{5}^{1-\text { loop }} & =-\frac{\mu}{6 \pi^{2} \zeta} .
\end{aligned}
$$

Although the overall sign of $\xi_{5}$ depends on the chirality, the relative negative sign compared to the static value of chiral magnetic conductivity $\sigma_{\chi}(0)=\mu / 4 \pi^{2}$ does not depend on chirality and is a meaningful dynamical result. Holographic computations produce the same negative sign between $\sigma_{\chi}(0)$ and $\xi_{5}$.

The above exercise shows quite a similar feature to what one finds in the electric conductivity, and one can follow the lessons we have learned from the computation of electric conductivity. The $1 / \zeta$ dependence from a pair of pinching propagators $S_{r a}(p) S_{a r}(p)$ signals a nonanalytic dependence on the coupling constant. In a multiloop ladder diagram shown, for example, in Fig. 2, each pair of pinching propagators sharing the same momentum produces a factor of $1 / \zeta \sim 1 /\left(g^{2} \log (1 / g) T\right)$ that compensates a $g^{2}$ from an extra gauge boson exchange, making the diagram of the same order as the one-loop diagram in the power counting of the coupling constant. Hence, one needs to sum up all multiloop ladder diagrams to get a correct leading order result for $\xi_{5}$; this can be achieved by solving a Schwinger-Dyson-type integral equation, which we will describe in the next section. More elaborate power counting $[58,60]$ shows that the leading contribution comes from the soft region of gauge boson momentum $Q \sim g T$, so one needs a HTL-resummed gauge boson propagator $[61,62]$ for the internal gauge boson 


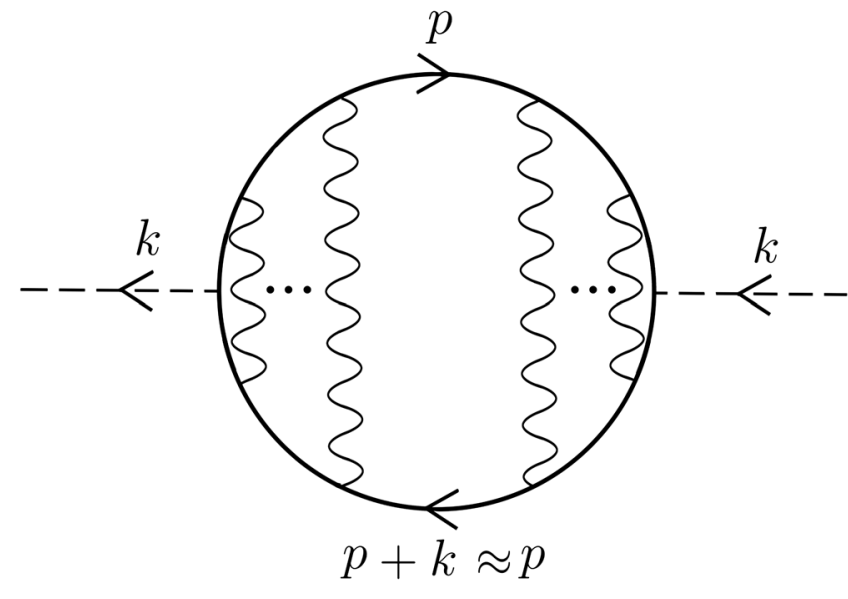

FIG. 2. A generic ladder diagram that contributes to the leading $\log$ result.

exchange lines. The fermion momentum stays hard $\sim T$, so fermion lines and all vertices are bare ones.

From the one-loop result of $\xi_{5}^{1-\text { loop }}$ with $1 / \zeta \sim$ $1 /\left(g^{2} \log (1 / g) T\right)$ dependence, the leading $\log$ result for $\xi_{5}$ from solving the Schwinger-Dyson equation might be expected to be $\sim 1 /\left(g^{2} \log (1 / g) T\right)$. However, the correct dependence turns out to be $\sim 1 /\left(g^{4} \log (1 / g) T\right)$; this is the same as in the electric conductivity. In both cases, a physics reason behind this is that small angle scatterings $(\theta \ll g)$ by transverse spacelike thermal gauge boson excitations (whose nonzero thermal spectral density is due to Landau damping physics) cannot affect the charge transport phenomena much, since they deflect charged fermion trajectories responsible for charge transports only slightly by small angles. On the other hand, these small angle scatterings by ultrasoft $\left(p \sim g^{2} T\right)$ transverse gauge bosons is the dominant source for the total decay rate $\zeta \sim g^{2} \log (1 / g) T$, where the $\log$ comes from $\log \left(m_{D} / \Lambda_{\mathrm{IR}}\right) \sim \log (1 / g)$ with $\Lambda_{\mathrm{IR}} \sim g^{2} T$ being the nonperturbative IR cutoff for the transverse magnetic sector, and $m_{D} \sim g T$ is the characteristic soft scale. This means that the effective IR regulator for the pinch singularities that is meaningful for the final conductivities is not given by the total damping rate $\zeta$, but is provided by larger angle scatterings $(\theta \gg g)$ and fermion conversion to gauge bosons, which are governed by the $g^{4} \log (1 / g) T$ rate. In the latter, the origin of the log is completely different: it is from $\log \left(T / m_{D}\right) \sim \log (1 / g)$. In our diagrammatic approach of the Schwinger-Dyson equation, this physics manifests itself in a nice cancellation of the leading $\log$ part of $\zeta$ in the equation that we will see in the following sections; what remains is indeed something of $g^{4} \log (1 / g) T$ coming from the rate of fermion conversion to gauge boson.

We end this section by recalling that the situation is quite different for color conductivity, where even small angle scatterings by thermal transverse gluons can change the color charge of charge carriers (either fermion or gauge boson) due to the non-Abelian nature of color charges [63]; thus, the same rate responsible for the leading log damping rate also governs the color conductivity, leading to its $1 /\left(g^{2} \log (1 / g)\right)$ behavior [64,65].

\section{LADDER RESUMMATION OF P-ODD PINCH SINGULARITIES IN $R A$ BASIS}

In this section, we set up the Schwinger-Dyson equation that sums up all-loop ladder diagrams with leading order pinch singularities. The idea is essentially similar to the one in the diagrammatic computations of shear viscosity or electric conductivity [58,59], except that we have to keep a finite external momentum $\boldsymbol{k}$ up to first order in $\boldsymbol{k}$ to extract a P-odd part [however, we can still set $\omega=0$ from the outset since one factor of $\omega$ comes out from kinematics, see (2.14) and (2.15)]. We choose to work in the real-time SchwingerKeldysh formalism in $r a$ basis for our convenience, rather than the Euclidean formalism with subsequent analytic continuation as used in some previous literature. For the electrical conductivity, we check that the correct result is obtained with this formalism.

As we are computing $G_{(r a)}^{i j}(k)$, the vertex at the far right in any ladder diagram is an a type, with one fermion leg $r$ type and the other a type. Since there is no aa propagator, the a-type leg should have an r-type leg in the other end on its left. Because a pinch singularity can appear only from a pair of $S_{r a}$ and $S_{a r}$, the r-type leg from the vertex should have an r-type leg on the other end on its left, since having a-type on the other end gives the same type of fermion propagator to the one from the former, and does not give a pinch singularity. See Fig. 3 for an exemplary ladder diagram that can give a leading pinch singularity. In our convention, one reads $r a$ types of a fermion propagator along the reversed direction of its momentum arrow, which can be seen in Fig. 3. The reason why having a rr-type propagator in the diagram can give rise to a pinch singularity is the thermal relation (2.11),

$$
S_{r r}(p)=\left(\frac{1}{2}-n_{+}\left(p^{0}\right)\right)\left(S_{r a}(p)-S_{a r}(p)\right) .
$$

One can therefore choose either the $S_{r a}$ or $S_{a r}$ piece from $S_{r r}$ to obtain a pair of $S_{r a}$ and $S_{a r}$ that gives a pinch singularity. It is clear, then, that the rest of a ladder diagram on the left, other than the far right vertex, should have two final fermion legs of $r$ type on its right, in order to create a leading order pinch singularity: that is, it has to be an effective rr-type vertex. At one-loop order, this was automatic since it is a bare $J_{r}^{i}$ vertex. What we have to do is to sum up all-loop ladder diagrams for this effective rr vertex that appears on the left side of the diagram.

Denoting by $\Lambda^{i}(p, k)$ the resulting summed vertex, which is a $2 \times 2$ matrix acting on the spinor space, where $p$ is the loop momentum and $k$ is the (small) external momentum, the final $G_{(r a)}^{i j, \text { Pinch }}(k)$ is obtained from two possible Feynman diagrams in Fig. 4; note that they look similar to those in Fig. 1 except that the vertex on the left is now $\Lambda^{i}(p, k)$ instead of $\sigma^{i}$, 


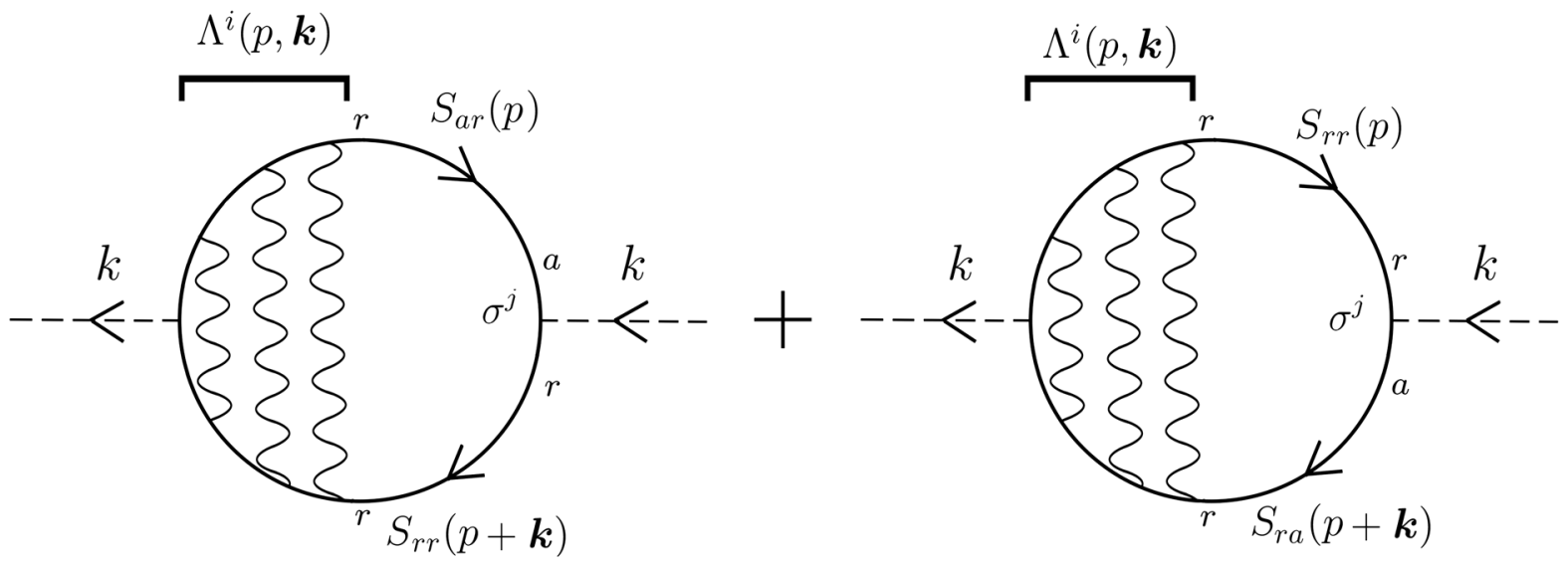

FIG. 3. Exemplar real time Feynman diagrams that can give leading pinch singularity.

$$
\begin{aligned}
G_{(r a)}^{i j, \text { Pinch }}(k)= & (-1) \int \frac{d^{4} p}{(2 \pi)^{4}} \operatorname{tr}\left[\Lambda ^ { i } ( p , k ) \left(S_{r a}(p+k) \sigma^{j} S_{r r}(p)\right.\right. \\
& \left.\left.+S_{r r}(p+k) \sigma^{j} S_{a r}(p)\right)\right]
\end{aligned}
$$

Using (3.1) and picking up only pairs of $S_{r a}$ and $S_{a r}$ for a pinch singularity, expanding it in $\omega$ with the same manipulation that led to (2.15), gives, up to first order in $\omega$,

$$
\begin{aligned}
& G_{(\text {ra })}^{i j, \text { Pinch }}(k) \\
& =-\omega \int \frac{d^{4} p}{(2 \pi)^{4}}\left(\frac{d n_{+}\left(p^{0}\right)}{d p^{0}}\right) \sum_{s= \pm} \\
& \quad \times \frac{\operatorname{tr}\left[\Lambda^{i}(p, \boldsymbol{k}) \mathcal{P}_{s}(\boldsymbol{p}+\boldsymbol{k}) \sigma^{j} \mathcal{P}_{s}(\boldsymbol{p})\right]}{\left(p^{0}-s|\boldsymbol{p}+\boldsymbol{k}|+i \zeta_{\boldsymbol{p}+\boldsymbol{k}, s} / 2\right)\left(p^{0}-s|\boldsymbol{p}|-i \zeta_{\boldsymbol{p}, s} / 2\right)},
\end{aligned}
$$

where we set external frequency $\omega \equiv k^{0}=0$ in the effective vertex $\Lambda^{i}(p, \boldsymbol{k})$ and other places since we already have one $\omega$ factor in front. Note also that the damping rate $\zeta_{p, s}$ depends on the on-shell momentum as well as $s= \pm$ (that is, whether it is a particle or antiparticle) as indicated in the expression. ${ }^{4}$ We will be concerned only with this object $\Lambda^{i}(p, \boldsymbol{k})$ after setting $\omega=0$ in the following.

The summation of all multiloop ladder diagrams for this effective rr-type vertex, starting from the bare one $J_{r}^{i}=\psi_{r}^{\dagger} \sigma^{i} \psi_{r}$, can be achieved by solving the associated Schwinger-Dyson-type equation, which is depicted in Fig. 5. The "kernel," which is made of two internal fermion lines and one soft gauge boson (we call it a photon) exchange, can have three possible Feynman diagrams that can give a leading pinch singularity, as shown in Fig. 6. The resulting Schwinger-Dyson equation ${ }^{5}$ is written as (note that in the following we denote the QED coupling constant by $e$ instead of $g$ )

$$
\begin{aligned}
\Lambda^{i}(p, \boldsymbol{k})= & \sigma^{i}+(i e)^{2} \int \frac{d^{4} Q}{(2 \pi)^{4}} \sigma^{\beta} S_{a r}(p+Q) \Lambda^{i}(p+Q, \boldsymbol{k}) S_{r a}(p+Q+\boldsymbol{k}) \sigma^{\alpha} G_{\alpha \beta}^{(r r)}(Q) \\
& +(i e)^{2} \int \frac{d^{4} Q}{(2 \pi)^{4}} \sigma^{\beta} S_{a r}(p+Q) \Lambda^{i}(p+Q, \boldsymbol{k}) S_{r r}(p+Q+\boldsymbol{k}) \sigma^{\alpha} G_{\alpha \beta}^{(a r)}(Q) \\
& +(i e)^{2} \int \frac{d^{4} Q}{(2 \pi)^{4}} \sigma^{\beta} S_{r r}(p+Q) \Lambda^{i}(p+Q, \boldsymbol{k}) S_{r a}(p+Q+\boldsymbol{k}) \sigma^{\alpha} G_{\alpha \beta}^{(r a)}(Q),
\end{aligned}
$$

where $G_{\alpha \beta}^{(a b)}(a, b=r$ or $a)$ are the photon propagators in Schwinger-Keldysh contour,

$$
\begin{aligned}
G_{\alpha \beta}^{(a b)} & \equiv\left\langle A_{\alpha}^{(a)}(Q) A_{\beta}^{(b)}(-Q)\right\rangle_{\mathrm{SK}} \\
& =\int d^{4} x e^{-i Q x}\left\langle A_{\alpha}^{(a)}(x) A_{\beta}^{(b)}(0)\right\rangle_{\mathrm{SK}},
\end{aligned}
$$

\footnotetext{
${ }^{4}$ The dependence on $s$ comes via the combination $s \mu$ in the presence of the chemical potential $\mu$ that we are considering. See Appendix B for a detailed discussion.

${ }^{5}$ In [59] it was shown that the Ward identity requires the addition of an extra term in Fig. 5 involving soft fermion lines. This diagram gives a subleading contribution to the electric conductivity, and we expect the same for our $\xi_{5}$. We leave the explicit computation to a future work.
} 

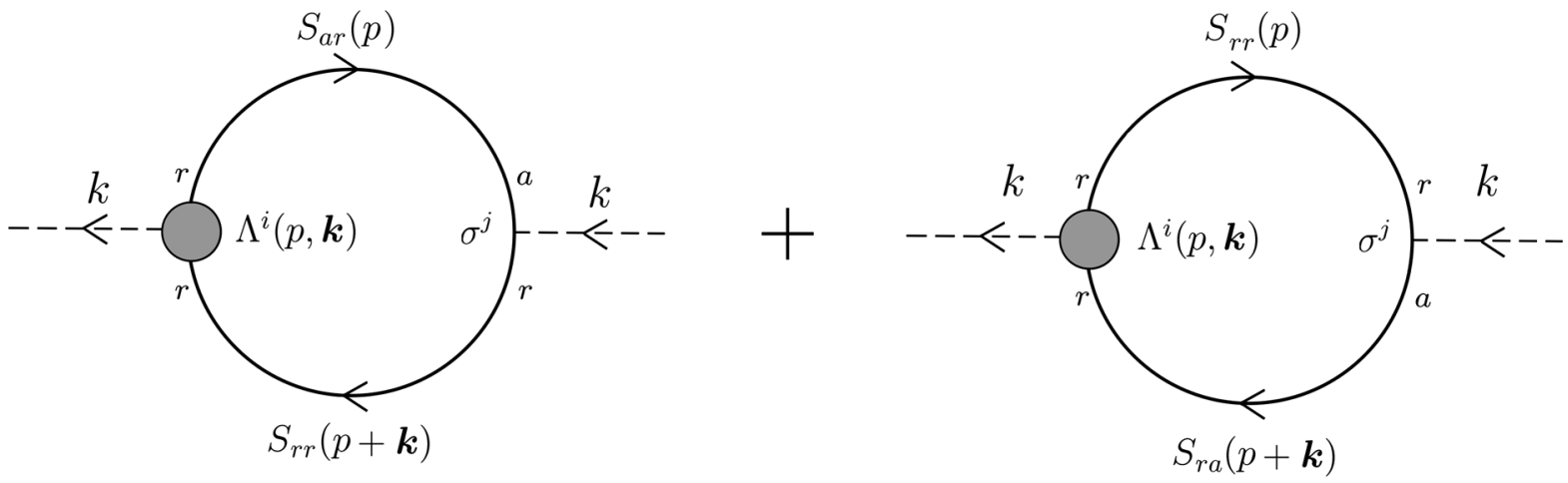

FIG. 4. The diagrams that need to be computed to obtain the retarded response function to leading log order. The effective vertex on the left includes an infinite number of ladder diagrams.

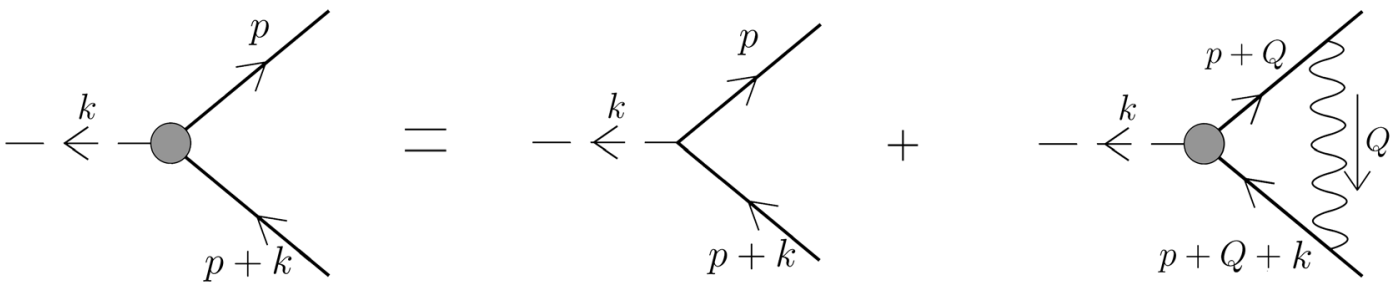

FIG. 5. The Schwinger-Dyson equation for the effective vertex $\Lambda^{i}(p, \boldsymbol{k})$.
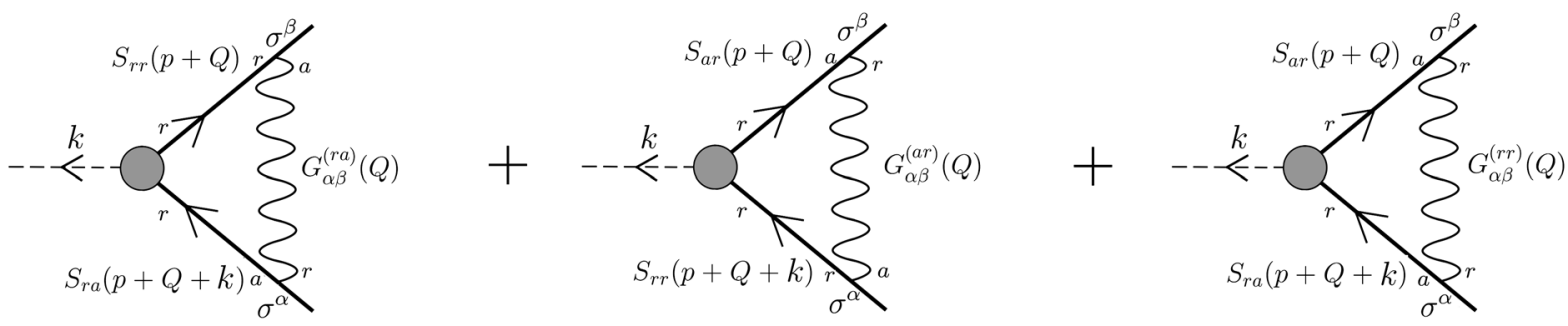

FIG. 6. The three real-time diagrams with leading pinch singularity for the kernel in the Schwinger-Dyson equation depicted in Fig. 5. The effective vertex connects only to r-type endings of fermion propagators.

including HTL photon self-energy (that is, $J J$ correlation functions) since the photon momentum $Q$ is soft. We will work in the Coulomb gauge, as it separates longitudinal and transverse modes in a clear way. A summary of $G_{\alpha \beta}^{(a b)}$ in this gauge, including the $\mathrm{P}$-odd part coming from the $\mathrm{P}$-odd part of HTL photon self-energy, is given in Appendix A, where we also find some useful sum rules for the P-odd part of their spectral density, which will be important for later use. Using (3.1) and a similar thermal relation for photons (see Appendix A),

$$
\begin{aligned}
G_{\alpha \beta}^{(r r)}(Q) & =\left(\frac{1}{2}+n_{B}\left(q^{0}\right)\right)\left(G_{\alpha \beta}^{(r a)}(Q)-G_{\alpha \beta}^{(a r)}(Q)\right) \\
& =\left(\frac{1}{2}+n_{B}\left(q^{0}\right)\right) \rho_{\alpha \beta}^{\mathrm{ph}}(Q)
\end{aligned}
$$

where the photon spectral density is defined by

$$
\rho_{\alpha \beta}^{\mathrm{ph}}(Q) \equiv\left(G_{\alpha \beta}^{(r a)}(Q)-G_{\alpha \beta}^{(a r)}(Q)\right)
$$

and $n_{B}\left(q^{0}\right)=1 /\left(e^{\beta q^{0}}-1\right)$, the pinch singularity part of the integral equation becomes

$$
\begin{aligned}
\Lambda^{i}(p, \boldsymbol{k})= & \sigma^{i}+(i e)^{2} \int \frac{d^{4} Q}{(2 \pi)^{4}}\left[\sigma^{\beta} S_{a r}(p+Q) \Lambda^{i}\right. \\
& \times(p+Q, \boldsymbol{k}) S_{r a}(p+Q+\boldsymbol{k}) \sigma^{\alpha} \\
& \left.\times \rho_{\alpha \beta}^{\mathrm{ph}}(Q)\left(n_{+}\left(p^{0}+q^{0}\right)+n_{B}\left(q^{0}\right)\right)\right] .
\end{aligned}
$$

Note that the photon spectral density $\rho_{\alpha \beta}^{\mathrm{ph}}(Q)$ is Hermitian in $(\alpha, \beta)$ indices, but not necessarily real. In fact, the P-odd self-energy leads to a purely imaginary, antisymmetric contribution to the spectral density. We refer the reader to Appendix A for a detailed exposition. 
From the pair $S_{a r}(p+Q) S_{r a}(p+Q+\boldsymbol{k})$ in (3.8) for the small $\boldsymbol{k}$ limit, one can extract the leading pinch singularity,

$$
\begin{aligned}
& S_{a r}(p+Q) \Lambda^{i}(p+Q, \boldsymbol{k}) S_{r a}(p+Q+k) \\
& \quad \rightarrow \sum_{t= \pm} \frac{i^{2} \mathcal{P}_{t}(\boldsymbol{p}+\boldsymbol{q}) \Lambda^{i}(p+Q, \boldsymbol{k}) \mathcal{P}_{t}(\boldsymbol{p}+\boldsymbol{q}+\boldsymbol{k})}{\left(p^{0}+q^{0}-t|\boldsymbol{p}+\boldsymbol{q}|-i \zeta_{\boldsymbol{p}+\boldsymbol{q}, t} / 2\right)\left(p^{0}+q^{0}-t|\boldsymbol{p}+\boldsymbol{q}+\boldsymbol{k}|+i \zeta_{\boldsymbol{p}+\boldsymbol{q}+\boldsymbol{k}, t} / 2\right)} .
\end{aligned}
$$

Because the photon momentum $Q$ is soft, $Q \ll|\boldsymbol{p}| \sim T$, and the pinch singularity in the final equation for $G_{(r a)}^{i j \text { Pinch }}(k)$ in (3.3) necessitates the loop momentum $p$ to be on-shell, $p^{0}=s|\boldsymbol{p}|$, the only possible way to have a pinch singularity for soft $q^{0}$ integration in (3.8) is to choose only the $t=s$ piece in the expression (3.9). This means that in a ladder diagram the leading pinch singular contribution comes from a particle loop or an antiparticle loop without any "transition" between particle and antiparticle throughout the diagram. Physically, it is obvious that a nearly on-shell particle (antiparticle) cannot change to an antiparticle (particle) with soft photon scatterings. Therefore, for a given choice of $s$ in (3.3), we keep only the $t=s$ piece of (3.9) in the integral equation (3.8), and the solution of the resulting integral equation we also label by $s: \Lambda_{s}(p, \boldsymbol{k})$. The more correct expression for (3.3) is then

$$
G_{(r a)}^{i j, \text { Pinch }}(k)=-\omega \int \frac{d^{4} p}{(2 \pi)^{4}}\left(\frac{d n_{+}\left(p^{0}\right)}{d p^{0}}\right) \sum_{s= \pm} \frac{\operatorname{tr}\left[\Lambda_{s}^{i}(p, \boldsymbol{k}) \mathcal{P}_{s}(\boldsymbol{p}+\boldsymbol{k}) \sigma^{j} \mathcal{P}_{s}(\boldsymbol{p})\right]}{\left(p^{0}-s|\boldsymbol{p}+\boldsymbol{k}|+i \zeta_{\boldsymbol{p}+\boldsymbol{k}, s} / 2\right)\left(p^{0}-s|\boldsymbol{p}|-i \zeta_{\boldsymbol{p}, s} / 2\right)},
$$

where $\Lambda_{s}^{i}(p, \boldsymbol{k})$ satisfies the integral equation

$$
\begin{aligned}
\Lambda_{s}^{i}(p, \boldsymbol{k})= & \sigma^{i}+e^{2} \int \frac{d^{4} Q}{(2 \pi)^{4}}\left[\sigma^{\beta} \mathcal{P}_{s}(\boldsymbol{p}+\boldsymbol{q}) \Lambda_{s}^{i}(p+Q, \boldsymbol{k}) \mathcal{P}_{s}(\boldsymbol{p}+\boldsymbol{q}+\boldsymbol{k}) \sigma^{\alpha}\right. \\
& \left.\times \frac{\rho_{\alpha \beta}^{\mathrm{ph}}(Q)\left(n_{+}\left(p^{0}+q^{0}\right)+n_{B}\left(q^{0}\right)\right)}{\left(p^{0}+q^{0}-s|\boldsymbol{p}+\boldsymbol{q}|-i \zeta_{\boldsymbol{p}+\boldsymbol{q}, s} / 2\right)\left(p^{0}+q^{0}-s|\boldsymbol{p}+\boldsymbol{q}+\boldsymbol{k}|+i \zeta_{\boldsymbol{p}+\boldsymbol{q}+\boldsymbol{k}, s} / 2\right)}\right] .
\end{aligned}
$$

The rest of the paper will focus on solving the integral equation (3.11) in leading logarithmic order in the coupling constant $e$.

Because our transport coefficient $\xi_{5}$ is obtained from the P-odd part of $G_{(r a)}^{i j, \text { Pinch }}(k)$ via

$$
G_{(r a)}^{i j, \text { Pinch,P-odd }}(\omega, \boldsymbol{k})=i \omega \xi_{5} \epsilon^{i j l} k^{l}+\mathcal{O}\left(\omega^{2}, \boldsymbol{k}^{2}\right),
$$

we would like to expand (3.10) in $\boldsymbol{k}$ up to first order, focusing only on the P-odd $\epsilon^{i j l} k^{l}$ structure at the same time. Since $\xi_{5}$ is $C P$-odd, and shares the same quantum number with the (axial) chemical potential $\mu$, it can only contain odd powers in $\mu$, as seen in the one-loop computation in the preceding section. In our work, we will only compute $\xi_{5}$ up to linear order in $\mu$ in the small- $\mu$ limit, neglecting higher order terms of $\mu^{3}$ and beyond. Therefore, we will only be interested in a linear $\mu$ dependence of (3.10) and (3.11) in the following.

\section{AN IMPORTANT SIMPLIFICATION}

In solving (3.11) up to linear order in $\boldsymbol{k}$, and using it to compute (3.10), there are various sources of $\boldsymbol{k}$ dependence appearing in the equations. The problematic source is the $\boldsymbol{k}$ dependence in the denominators of the equations (3.10) and (3.11). For example, in (3.10), we have

$$
\begin{aligned}
& p^{0}-s|\boldsymbol{p}+\boldsymbol{k}|+i \zeta_{\boldsymbol{p}+\boldsymbol{k}, s} / 2 \\
& \quad \approx p^{0}-s|\boldsymbol{p}|+i \zeta_{\boldsymbol{p}, s} / 2-s \hat{\boldsymbol{p}} \cdot \boldsymbol{k}+i\left(\partial \zeta_{\boldsymbol{p}, s} / \partial \boldsymbol{p}\right) \cdot \boldsymbol{k} / 2+\cdots,
\end{aligned}
$$

giving rise to, up to linear order in $\boldsymbol{k}$,

$$
\begin{aligned}
& \frac{1}{p^{0}-s|\boldsymbol{p}+\boldsymbol{k}|+i \zeta_{\boldsymbol{p}+\boldsymbol{k}, s} / 2} \\
& \quad \approx \frac{1}{p^{0}-s|\boldsymbol{p}|+i \zeta_{\boldsymbol{p}, s} / 2}+\frac{s \hat{\boldsymbol{p}} \cdot \boldsymbol{k}-i\left(\partial \zeta_{\boldsymbol{p}, s} / \partial \boldsymbol{p}\right) \cdot \boldsymbol{k} / 2}{\left(p^{0}-s|\boldsymbol{p}|+i \zeta_{\boldsymbol{p}, s} / 2\right)^{2}}+\cdots
\end{aligned}
$$

The second term is a double pole, and when used in (3.10) it engenders a $\sim 1 / \zeta^{2}$ dependence, which is larger than the usual $1 / \zeta$ pinch singularity. The same is true for the $\boldsymbol{k}$ dependence in the denominators of the integral equation (3.11). For the P-even part, this may be what one encounters when trying to include a finite $\boldsymbol{k}$ in the current correlation functions, which seems to be related to the expected appearance of diffusion pole structure

$$
\frac{\sigma_{e l}}{\omega-i D k^{2}} \approx \frac{\sigma_{e l}}{\omega}+i \sigma_{e l} D \frac{k^{2}}{\omega^{2}}+\cdots,
$$




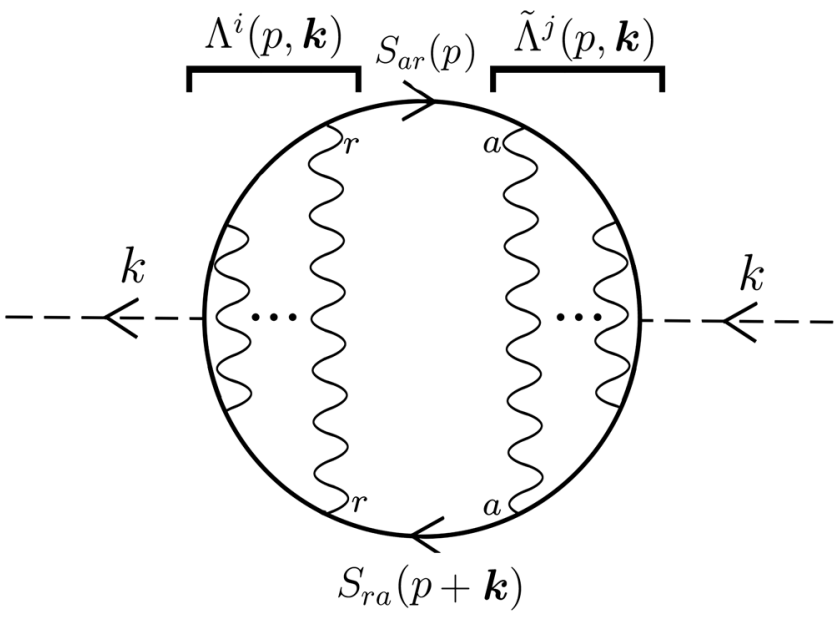

FIG. 7. Generic ladder diagram that gives rise to a leading log contribution. The ladders can be codified in effective vertices $\Lambda, \Lambda^{\prime}$.

since the Einstein relation gives $D=\sigma_{e l} / \chi(\chi$ is the charge susceptibility) and the $\boldsymbol{k}$ dependence is quadratic in electric conductivity, $\sigma_{e l} \sim T^{3} / e^{4} \log (1 / e)$. However, such a diffusion pole structure is not expected in the P-odd part that we are interested in [12], ${ }^{6}$ and it is natural to expect that these $\boldsymbol{k}$ dependences from the denominators in (3.10) and (3.11) do not contribute to our P-odd structure $\epsilon^{i j l} k^{l}$. Let us show this important simplification in the following. As a consequence, one can ignore all $\boldsymbol{k}$ 's in the denominators of the equations, and the only interesting $\boldsymbol{k}$ dependence comes from the projection operators in the numerators.

Let us consider a generic multiloop ladder diagram depicted in Fig. 7, and let us choose an arbitrary internal $S_{r a} S_{a r}$ pair from the "side rail" that can give rise to a pinch singularity in the small $\boldsymbol{k}$ limit. By shifting the loop momentum $p$, the momentum that flows in $S_{r a}$ can be set as $p+\boldsymbol{k}$; then, the momentum of $S_{a r}$ is $p$. The denominator of $S_{r a}(p+\boldsymbol{k})$ contains a $\boldsymbol{k}$-linear piece as in (4.1). We would like to show that this $\boldsymbol{k}$ dependence does not lead to the P-odd structure $\epsilon^{i j l} k^{l}$.

Once we get a term like (4.2), that is linear in $\boldsymbol{k}$, from expanding the denominator of $S_{r a}(p+\boldsymbol{k})$, we should set $\boldsymbol{k}=0$ in all other parts of the diagram, since it already saturates the linear $\boldsymbol{k}$ dependence we are looking at. These include the projection operators in the numerators of $S_{r a}(p+\boldsymbol{k})$ and $S_{a r}(p), \mathcal{P}_{s}(\boldsymbol{p}+\boldsymbol{k})$ and $\mathcal{P}_{s}(\boldsymbol{p})$ (recall that we need to have the same $s$ throughout the diagram for the leading pinch singularity), as well as the remaining parts of the diagram other than the chosen $S_{r a}(p+\boldsymbol{k}) S_{a r}(p)$ pair,

\footnotetext{
${ }^{6} \mathrm{~A}$ diffusion pole structure in the P-odd part could arise if one considers the coupling to the energy-momentum sector of the theory, leading to "chiral magnetic energy flow." However, when expanded in $\boldsymbol{k}$ it would give a term of $\boldsymbol{k}^{3}$ or higher [66,67]. Also, the coupling to the energy-momentum sector is of order $\mu^{2}$, and the resulting $\mathrm{P}$-odd effect is of order $\mu^{3}[66,67]$. Therefore, we can ignore this possibility in our work.
}

which we call the effective vertices; let us call the part on the left $\Lambda^{i}(p, \boldsymbol{k})$ and the part on the right $\tilde{\Lambda}^{j}(p, \boldsymbol{k})$, see Fig. 7. The value of the diagram is then proportional to (the $p$ integral of)

$$
\operatorname{tr}\left[\Lambda^{i}(p) \mathcal{P}_{s}(\boldsymbol{p}) \tilde{\Lambda}^{j}(p) \mathcal{P}_{s}(\boldsymbol{p})\right] \propto \operatorname{tr}\left[\Lambda^{i}(p)\left(\overline{\boldsymbol{\sigma}} \cdot p_{s}\right) \tilde{\Lambda}^{j}(p)\left(\bar{\sigma} \cdot p_{s}\right)\right],
$$

where $\Lambda^{i}(p) \equiv \Lambda^{i}(p, \boldsymbol{k}=0), \tilde{\Lambda}^{j}(p) \equiv \tilde{\Lambda}^{j}(p, \boldsymbol{k}=0)$, etc. All $i j$ index structure comes from this spinor trace.

The effective vertex $\Lambda^{i}(p)$ is a $2 \times 2$ matrix in the spinor space; since $\sigma^{\mu}=(\mathbf{1}, \vec{\sigma})$ forms a basis for any $2 \times 2$ matrices, we write $\Lambda^{i}(p)=\sigma^{\mu} \lambda_{\mu}^{i}(p)$. By invoking rotational invariance, we generally have

$$
\begin{aligned}
& \lambda_{0}^{i}(p)=f_{0}\left(p^{0},|\boldsymbol{p}|\right) \boldsymbol{p}^{i}, \\
& \lambda_{l}^{i}(p)=f_{1}\left(p^{0},|\boldsymbol{p}|\right) \delta_{l}^{i}+f_{2}\left(p^{0},|\boldsymbol{p}|\right) \boldsymbol{p}_{l} \boldsymbol{p}^{i}+f_{3}\left(p^{0},|\boldsymbol{p}|\right) \epsilon_{l}^{i m} \boldsymbol{p}_{m},
\end{aligned}
$$

where $f_{i}$ are functions only on $p^{0}$ and $|\boldsymbol{p}|$. Similarly, we have for $\tilde{\Lambda}^{j}(p)=\sigma^{\nu} \tilde{\lambda}_{\nu}^{j}(p)$ with

$\tilde{\lambda}_{0}^{j}(p)=\tilde{f}_{0}\left(p^{0},|\boldsymbol{p}|\right) \boldsymbol{p}^{j}$,

$\tilde{\lambda}_{l}^{j}(p)=\tilde{f}_{1}\left(p^{0},|\boldsymbol{p}|\right) \delta_{l}^{j}+\tilde{f}_{2}\left(p^{0},|\boldsymbol{p}|\right) \boldsymbol{p}_{l} \boldsymbol{p}^{j}+\tilde{f}_{3}\left(p^{0},|\boldsymbol{p}|\right) \epsilon_{l}^{j m} \boldsymbol{p}_{m}$.

Inserting these representations of $\Lambda^{i}(p)$ and $\tilde{\Lambda}^{j}(p)$ into the above (4.4), and using the trace formula we repeat here,

$\operatorname{tr}\left[\sigma^{\mu} \bar{\sigma}^{\nu} \sigma^{\alpha} \bar{\sigma}^{\beta}\right]=2\left(g^{\mu \nu} g^{\alpha \beta}-g^{\mu \alpha} g^{\nu \beta}+g^{\mu \beta} g^{\nu \alpha}\right)+2 i \epsilon^{\mu \nu \alpha \beta}$,

we immediately see that the last $\epsilon$-tensor term in (4.7) does not contribute since $p_{s}$ appears twice in (4.4), and we have $\operatorname{tr}\left[\Lambda^{i}(p)\left(\bar{\sigma} \cdot p_{s}\right) \tilde{\Lambda}^{j}(p)\left(\bar{\sigma} \cdot p_{s}\right)\right]=4\left(\lambda^{i}(p) \cdot p_{s}\right)\left(\tilde{\lambda}^{j}(p) \cdot p_{s}\right)$,

where we use $p_{s}^{2}=0$. Then, we have

$$
\begin{aligned}
\lambda^{i}(p) \cdot p_{s} & =s|\boldsymbol{p}| \lambda_{0}^{i}(p)+\boldsymbol{p}^{l} \lambda_{l}^{i}(p) \\
& =\left(s|\boldsymbol{p}| f_{0}\left(p^{0},|\boldsymbol{p}|\right)+f_{1}\left(p^{0},|\boldsymbol{p}|\right)\right. \\
& \left.+|\boldsymbol{p}|^{2} f_{2}\left(p^{0},|\boldsymbol{p}|\right)\right) \boldsymbol{p}^{i},
\end{aligned}
$$

which is proportional to $\boldsymbol{p}^{i}$. Note that the piece involving $f_{3}$ drops out. The same conclusion is true, that is, $\tilde{\lambda}^{j}(p)$. $p_{s} \sim \boldsymbol{p}^{j}$; hence, the result for the trace in (4.4) is proportional to $\boldsymbol{p}^{i} \boldsymbol{p}^{j}$. Since it is symmetric with respect to $i j$, it is clear that the result cannot contribute to the P-odd part of $\epsilon^{i j l} k^{l}$. In summary, we have shown that $\boldsymbol{k}$ dependence from the denominator of any internal fermion line in leading pinch singularity limit does not contribute to the P-odd structure $\epsilon^{i j l} k^{l}$, and, hence, we can neglect all $\boldsymbol{k}$ 's appearing in the denominators, especially in our equations (3.10) and (3.11). 
Once we remove all $\boldsymbol{k}$ 's from the denominators, we have

$$
G_{(r a)}^{i j, \text { Pinch }}(k)=-\omega \int \frac{d^{4} p}{(2 \pi)^{4}}\left(\frac{d n_{+}\left(p^{0}\right)}{d p^{0}}\right) \sum_{s= \pm} \frac{\operatorname{tr}\left[\Lambda_{s}^{i}(p, \boldsymbol{k}) \mathcal{P}_{s}(\boldsymbol{p}+\boldsymbol{k}) \sigma^{j} \mathcal{P}_{s}(\boldsymbol{p})\right]}{\left(p^{0}-s|\boldsymbol{p}|+i \zeta_{\boldsymbol{p}, s} / 2\right)\left(p^{0}-s|\boldsymbol{p}|-i \zeta_{\boldsymbol{p}, s} / 2\right)},
$$

and

$$
\begin{aligned}
\Lambda_{s}^{i}(p, \boldsymbol{k})= & \sigma^{i}+e^{2} \int \frac{d^{4} Q}{(2 \pi)^{4}}\left[\sigma^{\beta} \mathcal{P}_{s}(\boldsymbol{p}+\boldsymbol{q}) \Lambda_{s}^{i}(p+Q, \boldsymbol{k}) \mathcal{P}_{s}(\boldsymbol{p}+\boldsymbol{q}+\boldsymbol{k}) \sigma^{\alpha}\right. \\
& \left.\times \frac{\rho_{\alpha \beta}^{\mathrm{ph}}(Q)\left(n_{+}\left(p^{0}+q^{0}\right)+n_{B}\left(q^{0}\right)\right)}{\left(p^{0}+q^{0}-s|\boldsymbol{p}+\boldsymbol{q}|-i \zeta_{\boldsymbol{p}+\boldsymbol{q}, s} / 2\right)\left(p^{0}+q^{0}-s|\boldsymbol{p}+\boldsymbol{q}|+i \zeta_{\boldsymbol{p}+\boldsymbol{q}, s} / 2\right)}\right] .
\end{aligned}
$$

The $p^{0}$ integration in (4.10) can be computed in the leading pinch singularity limit by replacing

$$
\frac{1}{\left(p^{0}-s|\boldsymbol{p}|+i \zeta_{\boldsymbol{p}, s} / 2\right)\left(p^{0}-s|\boldsymbol{p}|-i \zeta_{\boldsymbol{p}, s} / 2\right)} \rightarrow \frac{2 \pi}{\zeta_{\boldsymbol{p}, s}} \delta\left(p^{0}-s|\boldsymbol{p}|\right),
$$

which enforces the on-shell condition $p^{0}=s|\boldsymbol{p}|$ on the $p$ appearing in the integral equation (4.13). We will assume this onshell condition throughout our computation in the following sections. Then, the integral equation becomes

$$
\begin{aligned}
\Lambda_{s}^{i}(\boldsymbol{p}, \boldsymbol{k})= & \sigma^{i}+e^{2} \int \frac{d^{4} Q}{(2 \pi)^{4}}\left[\sigma^{\beta} \mathcal{P}_{s}(\boldsymbol{p}+\boldsymbol{q}) \Lambda_{s}^{i}(\boldsymbol{p}+\boldsymbol{q}, \boldsymbol{k}) \mathcal{P}_{s}(\boldsymbol{p}+\boldsymbol{q}+\boldsymbol{k}) \sigma^{\alpha}\right. \\
& \left.\times \frac{\rho_{\alpha \beta}^{\mathrm{ph}}(Q)\left(n_{+}\left(s|\boldsymbol{p}|+q^{0}\right)+n_{B}\left(q^{0}\right)\right)}{\left(q^{0}+s|\boldsymbol{p}|-s|\boldsymbol{p}+\boldsymbol{q}|-i \zeta_{\boldsymbol{p}+\boldsymbol{q}, s} / 2\right)\left(q^{0}+s|\boldsymbol{p}|-s|\boldsymbol{p}+\boldsymbol{q}|+i \zeta_{\boldsymbol{p}+\boldsymbol{q}, s} / 2\right)}\right],
\end{aligned}
$$

where $\left.\Lambda_{s}^{i}(\boldsymbol{p}, \boldsymbol{k}) \equiv \Lambda_{s}^{i}(p, \boldsymbol{k})\right|_{p^{0}=s|\boldsymbol{p}|}$. The reason why we can also replace $\Lambda_{s}^{i}(p+Q, \boldsymbol{k})$ in the integral kernel with its on-shell value $\Lambda_{s}^{i}(\boldsymbol{p}+\boldsymbol{q}, \boldsymbol{k})$ is that the pinch singularity in the kernel of the integral equation

$$
\frac{1}{\left(p^{0}+q^{0}-s|\boldsymbol{p}+\boldsymbol{q}|-i \zeta_{\boldsymbol{p}+\boldsymbol{q}, s} / 2\right)\left(p^{0}+q^{0}-s|\boldsymbol{p}+\boldsymbol{q}|+i \zeta_{\boldsymbol{p}+\boldsymbol{q}, s} / 2\right)} \rightarrow \frac{2 \pi}{\zeta_{\boldsymbol{p}+\boldsymbol{q}, s}} \delta\left(p^{0}+q^{0}-s|\boldsymbol{p}+\boldsymbol{q}|\right),
$$

will impose the on-shell condition $p^{0}+q^{0}=s|\boldsymbol{p}+\boldsymbol{q}|$ as well. With this replacement of (4.14) in (4.13), we finally have

$$
\begin{aligned}
\Lambda_{s}^{i}(\boldsymbol{p}, \boldsymbol{k})= & \sigma^{i}+e^{2} \int \frac{d^{4} Q}{(2 \pi)^{4}}\left[\sigma^{\beta} \mathcal{P}_{s}(\boldsymbol{p}+\boldsymbol{q}) \Lambda_{s}^{i}(\boldsymbol{p}+\boldsymbol{q}, \boldsymbol{k}) \mathcal{P}_{s}(\boldsymbol{p}+\boldsymbol{q}+\boldsymbol{k}) \sigma^{\alpha}\right. \\
& \left.\times \rho_{\alpha \beta}^{\mathrm{ph}}(Q)\left(n_{+}\left(s|\boldsymbol{p}|+q^{0}\right)+n_{B}\left(q^{0}\right)\right)(2 \pi) \delta\left(q^{0}+s|\boldsymbol{p}|-s|\boldsymbol{p}+\boldsymbol{q}|\right) / \zeta_{\boldsymbol{p}+\boldsymbol{q}, s}\right]
\end{aligned}
$$

which is our starting point in solving the integral equation in leading logarithmic order in the next section.

\section{LEADING LOG COMPUTATION}

The integral equation (4.15) obtained in the leading pinch singularity limit is a matrix equation, and it is desirable to transform it into a scalar equation. In fact, we do not need its full matrix structure: what we need at the end in (4.10) is the trace

$$
\begin{aligned}
& \operatorname{tr}\left[\Lambda_{s}^{i}(\boldsymbol{p}, \boldsymbol{k}) \mathcal{P}_{s}(\boldsymbol{p}+\boldsymbol{k}) \sigma^{j} \mathcal{P}_{s}(\boldsymbol{p})\right] \\
& \quad=\operatorname{tr}\left[\mathcal{P}_{s}(\boldsymbol{p}) \Lambda_{s}^{i}(\boldsymbol{p}, \boldsymbol{k}) \mathcal{P}_{s}(\boldsymbol{p}+\boldsymbol{k}) \sigma^{j}\right],
\end{aligned}
$$

and it is obvious that we only need the component of $\Lambda_{s}^{i}(\boldsymbol{p}, \boldsymbol{k})$ projected onto the eigenspace of the projection operator $\mathcal{P}_{s}(\boldsymbol{p})$ on the left and $\mathcal{P}_{s}(\boldsymbol{p}+\boldsymbol{k})$ on the right, that is,

$$
\mathcal{P}_{s}(\boldsymbol{p}) \Lambda_{s}^{i}(\boldsymbol{p}, \boldsymbol{k}) \mathcal{P}_{s}(\boldsymbol{p}+\boldsymbol{k}) .
$$

Since the spinor space is two dimensional, the above projected matrix is essentially a single number. This fact manifests itself by the following statement: for any $2 \times 2$ matrix $A$, the projected matrix $\mathcal{P}_{s}(\boldsymbol{p}) A \mathcal{P}_{s}(\boldsymbol{p}+\boldsymbol{k})$ must be proportional to the matrix $\mathcal{P}_{s}(\boldsymbol{p}) \mathcal{P}_{s}(\boldsymbol{p}+\boldsymbol{k})$ composed only of the projection operators. The proportionality constant, which contains the information on $A$, is easily found by comparing traces of the expected relation, 


$$
\begin{aligned}
\mathcal{P}_{s}(\boldsymbol{p}) A \mathcal{P}_{s}(\boldsymbol{p}+\boldsymbol{k}) & =\frac{\operatorname{tr}\left[\mathcal{P}_{s}(\boldsymbol{p}) A \mathcal{P}_{s}(\boldsymbol{p}+\boldsymbol{k})\right]}{\operatorname{tr}\left[\mathcal{P}_{s}(\boldsymbol{p}) \mathcal{P}_{s}(\boldsymbol{p}+\boldsymbol{k})\right]} \mathcal{P}_{s}(\boldsymbol{p}) \mathcal{P}_{s}(\boldsymbol{p}+\boldsymbol{k}) \\
& =\frac{\operatorname{tr}\left[\mathcal{P}_{s}(\boldsymbol{p}) A \mathcal{P}_{s}(\boldsymbol{p}+\boldsymbol{k})\right]}{\frac{1}{2}(1+\hat{\boldsymbol{p}} \cdot \widehat{\boldsymbol{p}+\boldsymbol{k}})} \mathcal{P}_{s}(\boldsymbol{p}) \mathcal{P}_{s}(\boldsymbol{p}+\boldsymbol{k}),
\end{aligned}
$$

where $\hat{\boldsymbol{p}} \equiv \boldsymbol{p} /|\boldsymbol{p}|, \quad$ and $\quad \operatorname{tr}\left[\mathcal{P}_{s}(\boldsymbol{p}) \mathcal{P}_{s}(\boldsymbol{p}+\boldsymbol{k})\right]=\frac{1}{2}(1+\hat{\boldsymbol{p}}$. $\boldsymbol{p}+\boldsymbol{k})$. Using this, it is straightforward to convert our matrix integral equation into a scalar equation; for this purpose, let us introduce three scalar functions $\mathcal{D}_{s}^{i}(\boldsymbol{p}, \boldsymbol{k}), \Sigma_{s}^{\mu}(\boldsymbol{p}, \boldsymbol{k})$, and $F_{s}(\boldsymbol{p} ; \boldsymbol{q}, \boldsymbol{k})$ by

$$
\begin{aligned}
& \mathcal{P}_{s}(\boldsymbol{p}) \Lambda_{s}^{i}(\boldsymbol{p}, \boldsymbol{k}) \mathcal{P}_{s}(\boldsymbol{p}+\boldsymbol{k}) \\
& \quad=\mathcal{D}_{s}^{i}(\boldsymbol{p}, \boldsymbol{k}) \mathcal{P}_{s}(\boldsymbol{p}) \mathcal{P}_{s}(\boldsymbol{p}+\boldsymbol{k}), \\
& \mathcal{P}_{s}(\boldsymbol{p}) \sigma^{\mu} \mathcal{P}_{s}(\boldsymbol{p}+\boldsymbol{k}) \\
& \quad=\Sigma_{s}^{\mu}(\boldsymbol{p}, \boldsymbol{k}) \mathcal{P}_{s}(\boldsymbol{p}) \mathcal{P}_{s}(\boldsymbol{p}+\boldsymbol{k}), \\
& \mathcal{P}_{s}(\boldsymbol{p}) \mathcal{P}_{s}(\boldsymbol{p}+\boldsymbol{q}) \mathcal{P}_{s}(\boldsymbol{p}+\boldsymbol{q}+\boldsymbol{k}) \mathcal{P}_{s}(\boldsymbol{p}+\boldsymbol{k}) \\
& \quad=F_{s}(\boldsymbol{p} ; \boldsymbol{q}, \boldsymbol{k}) \mathcal{P}_{s}(\boldsymbol{p}) \mathcal{P}_{s}(\boldsymbol{p}+\boldsymbol{k}) .
\end{aligned}
$$

The expressions for $\Sigma_{s}^{\mu}(\boldsymbol{p}, \boldsymbol{k})$ and $F_{s}(\boldsymbol{p} ; \boldsymbol{q}, \boldsymbol{k})$ can easily be found by computing the necessary traces involved; for example, we have

$$
\begin{aligned}
\Sigma_{s}^{0}(\boldsymbol{p}, \boldsymbol{k}) & =1, \Sigma_{s}^{i}(\boldsymbol{p}, \boldsymbol{k}) \\
& =\frac{s\left(\hat{\boldsymbol{p}}^{i}+(\widehat{\boldsymbol{p}+\boldsymbol{k}})^{i}\right)-i \epsilon^{i j} \hat{\boldsymbol{p}}_{j}(\widehat{\boldsymbol{p}+\boldsymbol{k}})_{l}}{(1+\hat{\boldsymbol{p}} \cdot \widehat{\boldsymbol{p}+\boldsymbol{k}})},
\end{aligned}
$$

and the expression for $F_{s}(\boldsymbol{p} ; \boldsymbol{q}, \boldsymbol{k})$ can be found in Appendix C. Using the fact that $\sigma^{\mu}$ is Hermitian, we also have

$$
\mathcal{P}_{s}(\boldsymbol{p}+\boldsymbol{k}) \sigma^{\mu} \mathcal{P}_{s}(\boldsymbol{p})=\left(\Sigma_{s}^{\mu}(\boldsymbol{p}, \boldsymbol{k})\right)^{*} \mathcal{P}_{s}(\boldsymbol{p}+\boldsymbol{k}) \mathcal{P}_{s}(\boldsymbol{p})
$$

The scalar function $\mathcal{D}_{s}^{i}(\boldsymbol{p}, \boldsymbol{k})$ is what we would like to find by solving the integral equation; once it is found, the final expression for the correlation function $G_{(r a)}^{i j, \text { Pinch }}(k)$ is given from (4.10) by

$$
\begin{aligned}
G_{(r a)}^{i j, \operatorname{Pinch}}(k) & =-\omega \int \frac{d^{4} p}{(2 \pi)^{4}}\left(\frac{d n_{+}\left(p^{0}\right)}{d p^{0}}\right) \sum_{s= \pm} \frac{\operatorname{tr}\left[\mathcal{P}_{s}(\boldsymbol{p}) \Lambda_{s}^{i}(\boldsymbol{p}, \boldsymbol{k}) \mathcal{P}_{s}(\boldsymbol{p}+\boldsymbol{k}) \sigma^{j}\right]}{\left(p^{0}-s|\boldsymbol{p}|+i \zeta_{\boldsymbol{p}, s} / 2\right)\left(p^{0}-s|\boldsymbol{p}|-i \zeta_{\boldsymbol{p}, s} / 2\right)} \\
& =-\omega \int \frac{d^{4} p}{(2 \pi)^{4}}\left(\frac{d n_{+}\left(p^{0}\right)}{d p^{0}}\right) \sum_{s= \pm} \frac{\mathcal{D}_{s}^{i}(\boldsymbol{p}, \boldsymbol{k}) \operatorname{tr}\left[\mathcal{P}_{s}(\boldsymbol{p}+\boldsymbol{k}) \sigma^{j} \mathcal{P}_{s}(\boldsymbol{p})\right]}{\left(p^{0}-s|\boldsymbol{p}|+i \zeta_{\boldsymbol{p}, s} / 2\right)\left(p^{0}-s|\boldsymbol{p}|-i \zeta_{\boldsymbol{p}, s} / 2\right)} \\
& =-\omega \int \frac{d^{4} p}{(2 \pi)^{4}}\left(\frac{d n_{+}\left(p^{0}\right)}{d p^{0}}\right) \sum_{s= \pm} \frac{\mathcal{D}_{s}^{i}(\boldsymbol{p}, \boldsymbol{k})\left(\Sigma_{s}^{j}(\boldsymbol{p}, \boldsymbol{k})\right)^{*} \frac{1}{2}(1+\hat{\boldsymbol{p}} \cdot \widehat{\boldsymbol{p}+\boldsymbol{k})}}{\left(p^{0}-s|\boldsymbol{p}|+i \zeta_{\boldsymbol{p}, s} / 2\right)\left(p^{0}-s|\boldsymbol{p}|-i \zeta_{\boldsymbol{p}, s} / 2\right)} \\
& =-\left.\omega \int \frac{d^{3} \boldsymbol{p}}{(2 \pi)^{3}} \sum_{s= \pm}\left(\frac{d n_{+}\left(p^{0}\right)}{d p^{0}}\right)\right|_{p^{0}=s|\boldsymbol{p}|}\left(\Sigma_{s}^{j}(\boldsymbol{p}, \boldsymbol{k})\right)^{*} \frac{\mathcal{D}_{s}^{i}(\boldsymbol{p}, \boldsymbol{k})}{\zeta_{\boldsymbol{p}, s}}
\end{aligned}
$$

where we use (5.4),(5.6) in the second and third lines, and replace $\frac{1}{2}(1+\hat{\boldsymbol{p}} \cdot \widehat{\boldsymbol{p}+\boldsymbol{k}})$ with unity in the last line, since $\hat{\boldsymbol{p}} \cdot \widehat{\boldsymbol{p}+\boldsymbol{k}}=1$ up to negligible $\mathcal{O}\left(\boldsymbol{k}^{2}\right)$ corrections. As the last expression involves the combination $\mathcal{D}_{s}^{i}(\boldsymbol{p}, \boldsymbol{k}) / \zeta_{\boldsymbol{p}, s}$, let us also define

$$
\phi_{s}^{i}(\boldsymbol{p}, \boldsymbol{k}) \equiv \frac{\mathcal{D}_{s}^{i}(\boldsymbol{p}, \boldsymbol{k})}{\zeta_{\boldsymbol{p}, s}}
$$

Applying projection operators $\mathcal{P}_{s}(\boldsymbol{p})$ and $\mathcal{P}_{s}(\boldsymbol{p}+\boldsymbol{k})$ on the left and right, respectively, to our integral equation (4.15), and using (5.4), (5.6), we finally obtain the following scalar integral equation to be solved for $\phi_{s}^{i}(\boldsymbol{p}, \boldsymbol{k})$ :

$$
\begin{aligned}
\zeta_{\boldsymbol{p}, s} \phi_{s}^{i}(\boldsymbol{p}, \boldsymbol{k})= & \Sigma_{s}^{i}(\boldsymbol{p}, \boldsymbol{k})+e^{2} \int \frac{d^{4} Q}{(2 \pi)^{4}} \Sigma_{s}^{\beta}(\boldsymbol{p}, \boldsymbol{q}) \phi_{s}^{i}(\boldsymbol{p}+\boldsymbol{q}, \boldsymbol{k})\left(\Sigma_{s}^{\alpha}(\boldsymbol{p}+\boldsymbol{k}, \boldsymbol{q})\right)^{*} F_{s}(\boldsymbol{p} ; \boldsymbol{q}, \boldsymbol{k}) \\
& \times \rho_{\alpha \beta}^{\mathrm{ph}}(Q)\left(n_{+}\left(s|\boldsymbol{p}|+q^{0}\right)+n_{B}\left(q^{0}\right)\right)(2 \pi) \delta\left(q^{0}+s|\boldsymbol{p}|-s|\boldsymbol{p}+\boldsymbol{q}|\right) \\
= & \Sigma_{s}^{i}(\boldsymbol{p}, \boldsymbol{k})+e^{2} \int \frac{d^{4} Q}{(2 \pi)^{4}} \mathcal{K}_{s}(\boldsymbol{p}, \boldsymbol{k} ; Q) \phi_{s}^{i}(\boldsymbol{p}+\boldsymbol{q}, \boldsymbol{k})(2 \pi) \delta\left(q^{0}+s|\boldsymbol{p}|-s|\boldsymbol{p}+\boldsymbol{q}|,\right.
\end{aligned}
$$

with an integral kernel $\mathcal{K}_{s}(\boldsymbol{p}, \boldsymbol{k} ; Q)$ defined as

$$
\mathcal{K}_{s}(\boldsymbol{p}, \boldsymbol{k} ; Q) \equiv \Sigma_{s}^{\beta}(\boldsymbol{p}, \boldsymbol{q})\left(\Sigma_{s}^{\alpha}(\boldsymbol{p}+\boldsymbol{k}, \boldsymbol{q})\right)^{*} \rho_{\alpha \beta}^{\mathrm{ph}}(Q) F_{s}(\boldsymbol{p} ; \boldsymbol{q}, \boldsymbol{k})\left(n_{+}\left(s|\boldsymbol{p}|+q^{0}\right)+n_{B}\left(q^{0}\right)\right) .
$$


Our task is to find a solution for $\phi_{s}^{i}(\boldsymbol{p}, \boldsymbol{k})$ up to first order in $\boldsymbol{k}$ that can give rise to the P-odd structure $\epsilon^{i j l} \boldsymbol{k}^{l}$ in the expression (5.7) for $G_{(r a)}^{i j \text { Pinch }}(k)$. We will be interested in only the leading logarithmic order in $e$.

The structure of $\phi_{s}^{i}(\boldsymbol{p}, \boldsymbol{k})$ expanded up to first order in $\boldsymbol{k}$ can be severely constrained by rotational invariance,

$$
\begin{aligned}
\phi_{s}^{i}(\boldsymbol{p}, \boldsymbol{k})= & \chi_{s}^{i}(\boldsymbol{p})+f_{s}^{i l}(\boldsymbol{p}) \boldsymbol{k}^{l} \\
= & \chi_{s}(|\boldsymbol{p}|) \hat{\boldsymbol{p}}^{i}+a_{s}(|\boldsymbol{p}|) \boldsymbol{k}^{i}+b_{s}(|\boldsymbol{p}|) \hat{\boldsymbol{p}}{ }^{i}(\hat{\boldsymbol{p}} \cdot \boldsymbol{k}) \\
& +i \frac{1}{|\boldsymbol{p}|} c_{s}(|\boldsymbol{p}|) \epsilon^{i l m} \hat{\boldsymbol{p}}^{l} \boldsymbol{k}^{m},
\end{aligned}
$$

with four scalar functions $\chi_{s}, a_{s}, b_{s}, c_{s}$ that depend only on $|\boldsymbol{p}|$. Using the expansion of $\left(\Sigma_{s}^{j}(\boldsymbol{p}, \boldsymbol{k})\right)^{*}$ up to first order in $\boldsymbol{k}$,

$$
\left(\Sigma_{s}^{j}(\boldsymbol{p}, \boldsymbol{k})\right)^{*}=s \hat{\boldsymbol{p}}^{j}+\frac{s}{2|\boldsymbol{p}|}\left(\delta^{j l}-\hat{\boldsymbol{p}}^{j} \hat{\boldsymbol{p}}^{l}\right) \boldsymbol{k}^{l}+\frac{i}{2|\boldsymbol{p}|} \epsilon^{j l m} \hat{\boldsymbol{p}}^{l} \boldsymbol{k}^{m},
$$

the expression for $G_{(\mathrm{ra})}^{i j \text { Pinch }}(k)$ from (5.7) can be computed to first order in $\boldsymbol{k}$ to find the $\mathrm{P}$-odd structure proportional to $\epsilon^{i j l} \boldsymbol{k}^{l}$ as

$$
\begin{aligned}
G_{(r a)}^{i j, \text { Pinch }}(k)= & -\left.\omega \int \frac{d^{3} \boldsymbol{p}}{(2 \pi)^{3}} \sum_{s= \pm}\left(\frac{d n_{+}\left(p^{0}\right)}{d p^{0}}\right)\right|_{p^{0}=s|\boldsymbol{p}|} \\
& \times\left(\sum_{s}^{j}(\boldsymbol{p}, \boldsymbol{k})\right)^{*} \phi_{s}^{i}(\boldsymbol{p}, \boldsymbol{k}) \\
\sim & -\left.\frac{i}{3} \omega \int \frac{d^{3} \boldsymbol{p}}{(2 \pi)^{3}} \sum_{s= \pm}\left(\frac{d n_{+}\left(p^{0}\right)}{d p^{0}}\right)\right|_{p^{0}=s|\boldsymbol{p}|} \frac{1}{|\boldsymbol{p}|} \\
& \times\left(s c_{s}(|\boldsymbol{p}|)-\frac{1}{2} \chi_{s}(|\boldsymbol{p}|)\right) \epsilon^{i j l} \boldsymbol{k}^{l},
\end{aligned}
$$

where the $\sim$ in the above expression is only concerned with P-odd terms. Note that the two functions $\left(a_{s}, b_{s}\right)$ do not contribute to our P-odd term, so we do not need to compute them. Therefore, in the following we will focus on $\chi_{s}$ and $c_{s}$ only.

\section{A. Computation of $\chi_{s}(|\boldsymbol{p}|)$}

The function $\chi_{s}(|\boldsymbol{p}|)$ has already been known in previous computations of electric conductivity, though we will see that there is an important correction to it when linear in $\mu$ that is relevant to our final value of $\xi_{5}$. It satisfies the integral equation (5.9) after setting $\boldsymbol{k}=0$,

$$
\begin{aligned}
\zeta_{\boldsymbol{p}, s} \chi_{s}(|\boldsymbol{p}|) \hat{\boldsymbol{p}}^{i}= & s \hat{\boldsymbol{p}}^{i}+e^{2} \int \frac{d^{4} Q}{(2 \pi)^{4}} \mathcal{K}_{s} \\
& \times(\boldsymbol{p}, 0 ; Q) \chi_{s}(|\boldsymbol{p}+\boldsymbol{q}|) \widehat{\boldsymbol{p}+\boldsymbol{q}^{i}} \\
& \times(2 \pi) \delta\left(q^{0}+s|\boldsymbol{p}|-s|\boldsymbol{p}+\boldsymbol{q}|\right) .
\end{aligned}
$$

Our treatment for $\chi_{s}(|\boldsymbol{p}|)$ that follows is mostly the same as that found in Refs. $[58,59]$, and our computation for $\chi_{s}$ will confirm previous results in the literature. Let us, however, present some details along with which we can introduce several key elements that will be needed in our next treatment for $c_{s}(|\boldsymbol{p}|)$, which is new and more to our interest.

First, it is important to observe that the integral that appears in the above equation,

$$
e^{2} \int \frac{d^{4} Q}{(2 \pi)^{4}} \mathcal{K}_{s}(\boldsymbol{p}, 0 ; Q)(2 \pi) \delta\left(q^{0}+s|\boldsymbol{p}|-s|\boldsymbol{p}+\boldsymbol{q}|\right),
$$

is precisely equal to the contribution to the fermion damping rate from soft photon scatterings at full order, which contains the leading $\sim e^{2} \log (1 / e) T$ part of the total damping rate. We reconfirm this fact explicitly in Appendix B including the P-odd spectral density of the soft photon propagator. Denoting this by $\zeta_{\boldsymbol{p}, s}^{\mathrm{sp}}$ (following the notation in Ref. [59]), and writing the total damping rate as $\zeta_{\boldsymbol{p}, s}=\zeta_{\boldsymbol{p}, s}^{\mathrm{sp}}+\zeta_{\boldsymbol{p}, s}^{\mathrm{sf}}$ where $\zeta_{\boldsymbol{p}, s}^{\mathrm{sf}}$ is the other remaining contribution to the damping rate arising from soft fermion scatterings (or, more precisely, hard fermions making converting to hard photons and soft fermions) which is of order $e^{4} \log (1 / e) T$, the integral equation (5.14) takes the form

$$
\begin{aligned}
& \zeta_{\boldsymbol{p}, s}^{\mathrm{sf}} \chi_{s}(|\boldsymbol{p}|) \hat{\boldsymbol{p}}^{i} \\
& =s \hat{\boldsymbol{p}}^{i}+e^{2} \int \frac{d^{4} Q}{(2 \pi)^{4}} \mathcal{K}_{s}(\boldsymbol{p}, 0 ; \boldsymbol{q}) \\
& \times\left[\chi_{s}(|\boldsymbol{p}+\boldsymbol{q}|) \widehat{\boldsymbol{p}+\boldsymbol{q}^{i}}-\chi_{s}(|\boldsymbol{p}|) \hat{\boldsymbol{p}}^{i}\right] \\
& \times(2 \pi) \delta\left(q^{0}+s|\boldsymbol{p}|-s|\boldsymbol{p}+\boldsymbol{q}|\right),
\end{aligned}
$$

where we no longer have $\zeta_{p, s}^{\mathrm{sp}} \sim e^{2} \log (1 / e) T$ explicitly in the equation, and what remains will be shown to be only of order $\sim e^{4} \log (1 / e) T$. This cancellation of $e^{2} \log (1 / e) T$ dependence due to the identity of (5.15) with $\zeta_{\boldsymbol{p}, s}^{\mathrm{sp}}$ is the diagrammatic manifestation of the physics discussion at the end of Sec. II: the relevant part of the damping rate that is responsible for fermionic charge transport phenomena is not the total damping rate governed by small-angle scatterings with ultrasoft transverse photons, but is the part arising from fermion conversions to photons with soft fermion scatterings, that is, $\zeta_{p, s}^{\text {sf }}$. In Appendix B, we present a computation of $\zeta_{p, s}^{\text {sf }}$ to leading log order [see Eq. (B48)], with special care for its $s \mu$ dependence, which we will need later.

Introducing a variable $z=\cos \varphi$ where $\varphi$ is the angle between $\boldsymbol{p}$ and $\boldsymbol{q}$, one can show that [59]

$$
\begin{aligned}
& \delta\left(q^{0}+s|\boldsymbol{p}|-s|\boldsymbol{p}+\boldsymbol{q}|\right) \\
& \quad=\left(\frac{|\boldsymbol{p}|+s q^{0}}{|\boldsymbol{p} \| \boldsymbol{q}|}\right) \delta\left(z-z_{0}\right) \Theta\left(|\boldsymbol{q}|^{2}-\left(q^{0}\right)^{2}\right),
\end{aligned}
$$


where $\Theta(x)$ is the Heaviside theta function, and

$$
z_{0}|\boldsymbol{q}|=\hat{\boldsymbol{p}} \cdot \boldsymbol{q}=s q^{0}+\frac{\left(q^{0}\right)^{2}-|\boldsymbol{q}|^{2}}{2|\boldsymbol{p}|} .
$$

Using this, one can transform the $Q$ integration into

$$
\begin{aligned}
& \int \frac{d^{4} Q}{(2 \pi)^{4}}(2 \pi) \delta\left(q^{0}+s|\boldsymbol{p}|-s|\boldsymbol{p}+\boldsymbol{q}|\right) \\
& \quad=\left.\int_{0}^{\infty} \frac{d|\boldsymbol{q} \| \boldsymbol{q}|}{(2 \pi)} \int_{-|\boldsymbol{q}|}^{|\boldsymbol{q}|} \frac{d q^{0}}{(2 \pi)}\left(1+\frac{s q^{0}}{|\boldsymbol{p}|}\right)\right|_{\hat{\boldsymbol{p}} \cdot \boldsymbol{q} \rightarrow s q^{0}+\frac{\left(q^{0}\right)^{2}-|q|^{2}}{2 \boldsymbol{p} \mid}},
\end{aligned}
$$

where one needs to replace any $\hat{\boldsymbol{p}} \cdot \boldsymbol{q}$ appearing in the integrand by $s q^{0}+\left(\left(q^{0}\right)^{2}-|\boldsymbol{q}|^{2}\right) /(2|\boldsymbol{p}|)$. As $Q=\left(q^{0}, \boldsymbol{q}\right) \sim$ $e T$ is soft and $\boldsymbol{p} \sim T$ is hard for the leading log contribution (which can be seen in retrospect), we expand the integrand in the integral equation (5.16) in powers of $\left(q^{0}, \boldsymbol{q}\right) /|\boldsymbol{p}|$. For example, after some algebra, we have up to $\mathcal{O}\left(Q^{2}\right)$

$$
\begin{aligned}
& \chi_{s}(|\boldsymbol{p}+\boldsymbol{q}|) \widehat{\boldsymbol{p}+\boldsymbol{q}}^{i}-\chi_{s}(|\boldsymbol{p}|) \hat{\boldsymbol{p}}^{i} \\
& \approx \hat{\boldsymbol{p}}^{i}\left(\frac{\left(q^{0}\right)^{2}-|\boldsymbol{q}|^{2}}{2|\boldsymbol{p}|^{2}} \chi_{s}(|\boldsymbol{p}|)+s q^{0} \chi_{s}^{\prime}(|\boldsymbol{p}|)+\frac{1}{2}\left(q^{0}\right)^{2} \chi_{s}^{\prime \prime}(|\boldsymbol{p}|)\right),
\end{aligned}
$$

where we use the replacement $\hat{\boldsymbol{p}} \cdot \boldsymbol{q} \rightarrow s q^{0}+\left(\left(q^{0}\right)^{2}-\right.$ $\left.|\boldsymbol{q}|^{2}\right) /(2|\boldsymbol{p}|)$ in the middle of the computation, and $\chi_{s}^{\prime}(x)=d \chi_{s}(x) / d x$, etc. Similarly, we need an expansion of $\mathcal{K}_{s}(\boldsymbol{p}, 0 ; \boldsymbol{q})$, as follows. With

$n_{+}\left(s|\boldsymbol{p}|+q^{0}\right)+n_{B}\left(q^{0}\right)=\frac{1}{\beta q^{0}}+s\left(n_{s}(|\boldsymbol{p}|)-\frac{1}{2}\right)+\mathcal{O}\left(q^{0}\right)$

and

$$
F_{s}(\boldsymbol{p} ; \boldsymbol{q}, 0)=\frac{1}{2}(1+\hat{\boldsymbol{p}} \cdot \widehat{\boldsymbol{p}+\boldsymbol{q}})=1+\mathcal{O}\left(\boldsymbol{q}^{2}\right),
$$

what remains in $\mathcal{K}_{s}(\boldsymbol{p}, 0 ; \boldsymbol{q})$ is the polarization-contracted photon spectral density

$$
\rho_{\alpha \beta}^{\mathrm{ph}}(Q)\left(\Sigma_{s}^{\alpha}(\boldsymbol{p}, \boldsymbol{q})\right)^{*} \Sigma_{s}^{\beta}(\boldsymbol{p}, \boldsymbol{q}),
$$

where we need to expand the polarization part $\left(\Sigma_{s}^{\alpha}(\boldsymbol{p}, \boldsymbol{q})\right)^{*} \Sigma_{s}^{\beta}(\boldsymbol{p}, \boldsymbol{q})$ up to first order in $\boldsymbol{q}$ for our leading $\log$ computation. In Coulomb gauge, $\rho_{0 i}^{\mathrm{ph}}(Q)=0$ $(i=1,2,3)$ and $\rho_{00}^{\mathrm{ph}}(Q) \equiv \rho_{L}(Q)$ is the longitudinal part of spectral density. The transverse part is

$$
\rho_{i j}^{\mathrm{ph}}(Q)=\rho_{T}(Q)\left(\delta_{i j}-\frac{\boldsymbol{q}^{i} \boldsymbol{q}^{j}}{|\boldsymbol{q}|^{2}}\right)+i \rho_{\mathrm{odd}}(Q) \epsilon^{i j l} \boldsymbol{q}_{l},
$$

where the second term is the P-odd contribution proportional to $\mu$ that arises from the P-odd part of currentcurrent correlation function (or photon self-energy) in the HTL limit, whose expression can be found in Appendix A. Note that it is purely imaginary, but antisymmetric in $i, j$, so it is a Hermitian matrix in $i, j$. For our purposes, we would only need its sum rules derived in Appendix A, Eqs. (A26) and (A27),

$$
\begin{aligned}
\int_{-|\boldsymbol{q}|}^{|\boldsymbol{q}|} \frac{d q^{0}}{(2 \pi)} \frac{1}{q^{0}} \rho_{\text {odd }}(Q) & =-\frac{e^{2} \mu}{(2 \pi)^{2}} \frac{1}{|\boldsymbol{q}|^{4}}+\cdots \\
\int_{-|\boldsymbol{q}|}^{|\boldsymbol{q}|} \frac{d q^{0}}{(2 \pi)} q^{0} \rho_{\text {odd }}(Q) & =\frac{0}{|\boldsymbol{q}|^{2}}+\cdots
\end{aligned}
$$

up to less singular terms in the small $|\boldsymbol{q}| \ll e T$ limit. All functions $\left(\rho_{L}, \rho_{T}, \rho_{\text {odd }}\right)$ are odd in $q^{0} \rightarrow-q^{0}$, so we need an extra odd power of $q^{0}$ in the final integrand to have a nonvanishing $q^{0}$ integral over $[-|\boldsymbol{q}|,+|\boldsymbol{q}|]$. The only $\mu$ dependence in the usual spectral densities $\rho_{L / T}(Q)$ is through the Debye mass, which is $m_{D}^{2}=e^{2} T^{2} / 6+$ $e^{2} \mu^{2} /\left(2 \pi^{2}\right)$ for a single Weyl fermion. Because we are looking at only up to linear $\mu$ dependence, we can safely neglect $\mu^{2}$ corrections in $m_{D}^{2}$ and use the $\mu=0$ results for $\rho_{L / T}(Q)$. After some algebra, we have up to $\mathcal{O}(\boldsymbol{q})$

$$
\begin{aligned}
& \rho_{\alpha \beta}^{\mathrm{ph}}(Q)\left(\Sigma_{s}^{\alpha}(\boldsymbol{p}, \boldsymbol{q})\right)^{*} \Sigma_{s}^{\beta}(\boldsymbol{p}, \boldsymbol{q}) \\
& \quad=\rho_{L}(Q)+\left(1-\frac{\left(q^{0}\right)^{2}}{|\boldsymbol{q}|^{2}}\right) \rho_{T}(Q)-\frac{s\left(\left(q^{0}\right)^{2}-|\boldsymbol{q}|^{2}\right)}{|\boldsymbol{p}|} \rho_{\text {odd }}(Q),
\end{aligned}
$$

where the last contribution from the P-odd spectral density, though quadratic in $\boldsymbol{q}$, is presented because its power counting is something new and different from those of $\rho_{L / T}(Q)$ as can be seen in (5.25), and should be checked carefully.

Let us first estimate this contribution from the P-odd spectral density in the integral equation (5.16). Collecting everything presented above, the contribution from the P-odd spectral density to the integral in (5.16) becomes

$$
\begin{aligned}
- & s \frac{e^{2}}{|\boldsymbol{p}|} \hat{\boldsymbol{p}}^{i} \int_{0}^{\infty} \frac{d|\boldsymbol{q} \| \boldsymbol{q}|}{(2 \pi)} \int_{-|\boldsymbol{q}|}^{|\boldsymbol{q}|} \frac{d q^{0}}{(2 \pi)}\left(1+\frac{s q^{0}}{|\boldsymbol{p}|}\right) \\
& \times\left(\frac{1}{\beta q^{0}}+s\left(n_{s}(|\boldsymbol{p}|)-\frac{1}{2}\right)\right)\left(\left(q^{0}\right)^{2}-|\boldsymbol{q}|^{2}\right) \\
& \times\left(\frac{\left(q^{0}\right)^{2}-|\boldsymbol{q}|^{2}}{2|\boldsymbol{p}|^{2}} \chi_{s}(|\boldsymbol{p}|)+s q^{0} \chi_{s}^{\prime}(|\boldsymbol{p}|)+\frac{1}{2}\left(q^{0}\right)^{2} \chi_{s}^{\prime \prime}(|\boldsymbol{p}|)\right) \\
& \times \rho_{\text {odd }}(Q),
\end{aligned}
$$


and, using the sum rules derived in Appendix A [(A26), (A27)], it is easy to find that the result is at most of order $\sim e^{4}$ without any logarithmic enhancement. Note that the $|\boldsymbol{q}|$ integration should have an UV cutoff $\sim T$ because we use a HTL approximation for soft momentum $Q \ll T$. In any case, these are of higher order than $e^{4} \log (1 / e)$, which is what we are interested in, and so can be neglected. Although we find that $\rho_{\text {odd }}(Q)$ does not affect the leading $\log$ equation for $\chi_{s}(|\boldsymbol{p}|)$, we will find shortly that it does give an important contribution to the equation for $c_{s}(|\boldsymbol{p}|)$ at leading $\log$, which is more to our interest.

The integral equation (5.16) then takes a form at leading $\log$ order as

$$
\begin{aligned}
\zeta_{\boldsymbol{p}, s}^{\mathrm{ff}} \chi_{s}(|\boldsymbol{p}|)= & s+e^{2} \int_{0}^{\infty} \frac{d|\boldsymbol{q} \| \boldsymbol{q}|}{(2 \pi)} \int_{-|\boldsymbol{q}|}^{|\boldsymbol{q}|} \frac{d q^{0}}{(2 \pi)}\left(1+\frac{s q^{0}}{|\boldsymbol{p}|}\right) \\
& \times\left(\frac{1}{\beta q^{0}}+s\left(n_{s}(|\boldsymbol{p}|)-\frac{1}{2}\right)\right) \\
& \times\left(\rho_{L}(Q)+\left(1-\frac{\left(q^{0}\right)^{2}}{|\boldsymbol{q}|^{2}}\right) \rho_{T}(Q)\right) \\
& \times\left(\frac{\left(q^{0}\right)^{2}-|\boldsymbol{q}|^{2}}{2|\boldsymbol{p}|^{2}} \chi_{s}(|\boldsymbol{p}|)+s q^{0} \chi_{s}^{\prime}(|\boldsymbol{p}|)\right. \\
& \left.+\frac{1}{2}\left(q^{0}\right)^{2} \chi_{s}^{\prime \prime}(|\boldsymbol{p}|)\right) .
\end{aligned}
$$

The remaining computation of various integrals of spectral densities is achieved at leading order using the well-known sum rules of $\rho_{L / T}(Q)$ [68]. The leading log contribution will come from the region $m_{D} \ll|\boldsymbol{q}| \lesssim T$; following the notations in Ref. [59] by defining

$$
J_{n}^{L / T} \equiv \int_{-|q|}^{|q|} \frac{d q^{0}}{(2 \pi)}\left(q^{0}\right)^{2 n-1} \rho_{L / T}(Q)
$$

we have for $|\boldsymbol{q}| \gg m_{D}$ the sum rules ${ }^{7}$

$$
\begin{aligned}
J_{0}^{L} \approx \frac{m_{D}^{2}}{|\boldsymbol{q}|^{4}}, & J_{0}^{T} \approx \frac{m_{D}^{2}}{4|\boldsymbol{q}|^{4}}\left(\log \frac{8|\boldsymbol{q}|^{2}}{m_{D}^{2}}-1\right), \\
J_{1}^{L} \approx \frac{m_{D}^{2}}{3|\boldsymbol{q}|^{2}}, & J_{1}^{T} \approx \frac{m_{D}^{2}}{4|\boldsymbol{q}|^{2}}\left(\log \frac{8|\boldsymbol{q}|^{2}}{m_{D}^{2}}-3\right), \\
J_{2}^{L} \approx \frac{m_{D}^{2}}{5}, & J_{2}^{T} \approx \frac{m_{D}^{2}}{4}\left(\log \frac{8|\boldsymbol{q}|^{2}}{m_{D}^{2}}-\frac{11}{3}\right) .
\end{aligned}
$$

Using these in (5.28), one encounters a logarithmic divergence

$$
\int_{m_{D}}^{T} \frac{d|\boldsymbol{q}|}{|\boldsymbol{q}|}=\log \left(T / m_{D}\right) \sim \log (1 / e)
$$

\footnotetext{
${ }^{7}$ The sum rules for the case $|\boldsymbol{q}| \ll m_{D}$ take different forms, and it can be checked that the ultrasoft region $|\boldsymbol{q}| \ll m_{D}$ does not give rise to logarithmic divergences.
}

where the IR cutoff is $m_{D}$, because the sum rule expressions used are valid only for $|\boldsymbol{q}| \gg m_{D}$ (see our footnote); the UV cutoff is $T$, as we assume soft $Q \ll T$ throughout our treatment, and therefore the modification for hard $Q$ will dampen away the UV divergences. Picking up the logarithmically enhanced terms in the integral, we finally obtain the differential equation

$$
\begin{aligned}
\zeta_{\boldsymbol{p}, s}^{\mathrm{sf}} \chi_{s}(|\boldsymbol{p}|)= & s-\frac{e^{2} m_{D}^{2} \log (1 / e)}{4 \pi}\left(\frac{1}{\beta|\boldsymbol{p}|^{2}} \chi_{s}(|\boldsymbol{p}|)\right. \\
& \left.-\left(\frac{1}{\beta|\boldsymbol{p}|}+n_{s}(|\boldsymbol{p}|)-\frac{1}{2}\right) \chi_{s}^{\prime}(|\boldsymbol{p}|)-\frac{1}{2 \beta} \chi_{s}^{\prime \prime}(|\boldsymbol{p}|)\right),
\end{aligned}
$$

which agrees with the known result in the literature for the case $\mu=0$. Note, however, the important $\mu$ dependence via $n_{s}(|\boldsymbol{p}|)$ in the differential equation for $\chi_{s}(|\boldsymbol{p}|)$,

$$
\begin{aligned}
n_{s}(|\boldsymbol{p}|) & =\frac{1}{e^{\beta(|\boldsymbol{p}|-s \mu)}+1} \\
& \approx \frac{1}{e^{\beta|\boldsymbol{p}|}+1}+s \beta \mu \frac{e^{\beta|\boldsymbol{p}|}}{\left(e^{\beta|\boldsymbol{p}|}+1\right)^{2}}+\mathcal{O}\left(\mu^{2}\right),
\end{aligned}
$$

as well as in $\zeta_{\boldsymbol{p}, s}^{\mathrm{sf}}$, which we compute in Appendix B [see Eq. (B48)],

$$
\begin{aligned}
\zeta_{\boldsymbol{p}, s}^{\mathrm{sf}}= & \frac{e^{2}}{4 \pi} \frac{m_{f}^{2} \log (1 / e)}{|\boldsymbol{p}|}\left(n_{B}(|\boldsymbol{p}|)+n_{-s}(0)\right) \\
& \approx \frac{e^{2}}{4 \pi} \frac{m_{f}^{2} \log (1 / e)}{|\boldsymbol{p}|}\left(n_{B}(|\boldsymbol{p}|)+\frac{1}{2}-s \frac{\beta \mu}{4}\right)+\mathcal{O}\left(\mu^{2}\right) .
\end{aligned}
$$

These give rise to an $s$-independent, linear-in- $\mu$ part in $\chi_{s}(|\boldsymbol{p}|)$ in addition to the usual $\mu$-independent part proportional to $s$. Here $m_{f}^{2}=\left(e^{2} / 4\right)\left(T^{2}+\mu^{2} / \pi^{2}\right)$ is the asymptotic thermal mass of fermions. The solution when expanded in $\mu$ then takes the form

$$
\chi_{s}(|\boldsymbol{p}|)=s \chi_{(0)}(|\boldsymbol{p}|)+\mu \chi_{(1)}(|\boldsymbol{p}|)+\mathcal{O}\left(\mu^{2}\right),
$$

and both $\chi_{(0)}$ and $\chi_{(1)}$ give separate contributions of the same leading order to the final expression for $\xi_{5}$ in (5.13). In fact, this $\mu$ dependence via $n_{s}(|\boldsymbol{p}|)$ and $\zeta_{\boldsymbol{p}, s}^{\mathrm{sf}}$ in (5.32) [i.e., the $\chi_{(1)}$ in Eq. (5.35)] also makes a contribution to the $\mu^{2}$ dependence of the electric conductivity in leading log order, which seems to have been missed in some previous literature. Our analysis in the above (with full expressions for $m_{D}^{2}$ and $m_{f}^{2}$ ) contains all the necessary elements that allow us to compute the full $\mu^{2}$ 
correction to electric conductivity; we present the correct computation of the $\mu^{2}$ correction to the electric conductivity in Appendix D.

\section{B. Computation of $c_{s}(|p|)$}

Let us next describe our analysis for $c_{s}(|\boldsymbol{p}|)$, which appears as the P-odd component of $\phi_{s}^{i}(\boldsymbol{p}, \boldsymbol{k}) \sim \phi_{s}^{i}(\boldsymbol{p}, 0)+$ $i\left(c_{s}(|\boldsymbol{p}|) /|\boldsymbol{p}|\right) \epsilon^{i l m} \hat{\boldsymbol{p}}^{l} \boldsymbol{k}^{m}$ when expanded in linear $\boldsymbol{k}$ [see (5.11)], and which satisfies our original integral equation (5.9) with a finite $\boldsymbol{k}$. Expanding the kernel function $\mathcal{K}_{s}(\boldsymbol{p}, \boldsymbol{k} ; Q)$ defined by (5.10) up to linear order in $\boldsymbol{k}$,

$$
\mathcal{K}_{s}(\boldsymbol{p}, \boldsymbol{k} ; Q)=\mathcal{K}_{s}(\boldsymbol{p}, 0 ; Q)+\mathcal{K}_{s}^{(1)}(\boldsymbol{p}, \boldsymbol{k} ; Q)+\mathcal{O}\left(\boldsymbol{k}^{2}\right),
$$

where $\mathcal{K}_{s}(\boldsymbol{p}, 0 ; Q)$ is something we have already used [see Eqs. (5.14) and (5.15)] to determine the zeroth order solution $\phi_{s}^{i}(\boldsymbol{p}, 0)=\chi_{s}(|\boldsymbol{p}|) \hat{\boldsymbol{p}}^{i}$, the part of the integral equation in (5.9) that is linear in $\boldsymbol{k}$ gives the integral equation for $c_{s}(|\boldsymbol{p}|)$. This takes the form

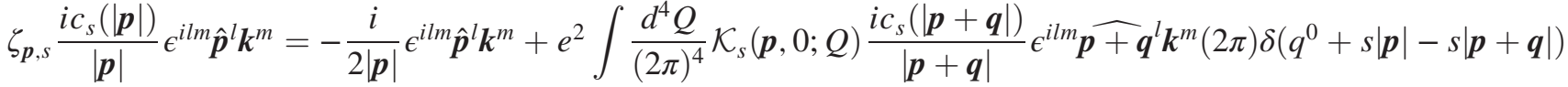

$$
\begin{aligned}
& +e^{2} \int \frac{d^{4} Q}{(2 \pi)^{4}} \mathcal{K}_{s}^{(1)}(\boldsymbol{p}, \boldsymbol{k} ; Q) \chi_{s}(|\boldsymbol{p}+\boldsymbol{q}|) \widehat{\boldsymbol{p}}^{i} \boldsymbol{q}^{i}(2 \pi) \delta\left(q^{0}+s|\boldsymbol{p}|-s|\boldsymbol{p}+\boldsymbol{q}|\right),
\end{aligned}
$$

where in the last term we understand that we extract only the P-odd term that has the same structure of $\epsilon^{i l m} \hat{\boldsymbol{p}}^{l} \boldsymbol{k}^{m}$. The first term on the right arises from the fact that $\Sigma_{s}^{i}(\boldsymbol{p}, \boldsymbol{k})$ as given by (5.5) contains the P-odd term when expanded linear in $\boldsymbol{k}$,

$$
\sum_{s}^{i}(\boldsymbol{p}, \boldsymbol{k}) \sim-\frac{i}{2|\boldsymbol{p}|} \epsilon^{i l m} \hat{\boldsymbol{p}}^{l} \boldsymbol{k}^{m}
$$

As before, it is important to use the fact that the integral

$$
e^{2} \int \frac{d^{4} Q}{(2 \pi)^{4}} \mathcal{K}_{s}(\boldsymbol{p}, 0 ; Q)(2 \pi) \delta\left(q^{0}+s|\boldsymbol{p}|-s|\boldsymbol{p}+\boldsymbol{q}|\right),
$$

which appears in the second term on the right side, is precisely equal to the contribution to the fermion damping rate arising from soft photon scatterings, $\zeta_{\boldsymbol{p}, s}^{\mathrm{sp}}$. Therefore, one can transform the above integral equation into the form

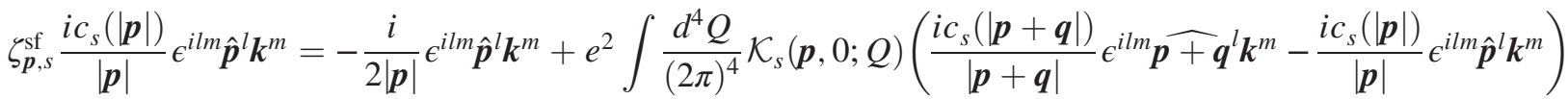

$$
\begin{aligned}
& \times(2 \pi) \delta\left(q^{0}+s|\boldsymbol{p}|-s|\boldsymbol{p}+\boldsymbol{q}|\right)+e^{2} \int \frac{d^{4} Q}{(2 \pi)^{4}} \mathcal{K}_{s}^{(1)}(\boldsymbol{p}, \boldsymbol{k} ; Q) \chi_{s}(|\boldsymbol{p}+\boldsymbol{q}|){\widehat{\boldsymbol{p}}+\boldsymbol{q}^{i}}^{i}(2 \pi) \delta\left(q^{0}\right. \\
& +s|\boldsymbol{p}|-s|\boldsymbol{p}+\boldsymbol{q}|)
\end{aligned}
$$

where the $\zeta_{p, s}^{\text {sf }}$ that appears on the left is the damping rate contribution arising from soft fermion scatterings only, which is of order $e^{4} \log (1 / e)$ rather than $e^{2} \log (1 / e)$.

The computation of the first integral on the right side of (5.40) at leading log order is almost identical to that of the previous integral in (5.16). Expanding up to quadratic order in $Q$, with the replacement $q^{l} \rightarrow(\hat{\boldsymbol{p}} \cdot \boldsymbol{q}) \hat{\boldsymbol{p}}^{l}$ due to rotational invariance of the $\boldsymbol{q}$ integral, we get, after some algebra,

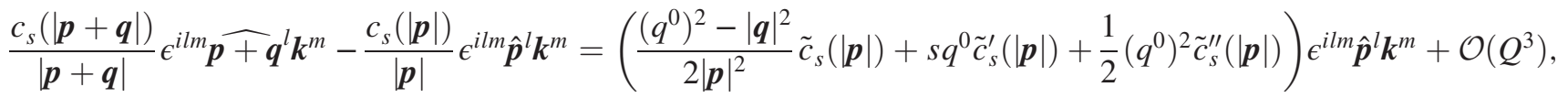

where $\tilde{c}_{s}(x) \equiv c_{s}(x) / x$. Comparing this with the previous expansion (5.20) for $\chi_{s}(|\boldsymbol{p}|)$, we find the identical structure appearing; therefore, we can simply use the previous result of the integral in (5.16) [see Eq. (5.32)] and replace $\chi_{s}$ with $\tilde{c}_{s}(x)=c_{s}(x) / x$ to get 


$$
\begin{aligned}
& e^{2} \int \frac{d^{4} Q}{(2 \pi)^{4}} \mathcal{K}_{s}(\boldsymbol{p}, 0 ; Q)\left(\frac{i c_{s}(|\boldsymbol{p}+\boldsymbol{q}|)}{|\boldsymbol{p}+\boldsymbol{q}|} \epsilon^{i l m} \widehat{\boldsymbol{p}+\boldsymbol{q}} \boldsymbol{k}^{m}-\frac{i c_{s}(|\boldsymbol{p}|)}{|\boldsymbol{p}|} \epsilon^{i l m} \hat{\boldsymbol{p}}^{l} \boldsymbol{k}^{m}\right)(2 \pi) \delta\left(q^{0}+s|\boldsymbol{p}|-s|\boldsymbol{p}+\boldsymbol{q}|\right) \\
& =-i \frac{e^{2} m_{D}^{2} \log (1 / e)}{4 \pi}\left(\frac{1}{\beta|\boldsymbol{p}|^{2}} \tilde{c}_{s}(|\boldsymbol{p}|)-\left(\frac{1}{\beta|\boldsymbol{p}|}+n_{s}(|\boldsymbol{p}|)-\frac{1}{2}\right) \tilde{c}_{s}^{\prime}(|\boldsymbol{p}|)-\frac{1}{2 \beta} \tilde{c}_{s}^{\prime \prime}(|\boldsymbol{p}|)\right) \epsilon^{i l m} \hat{\boldsymbol{p}}^{l} \boldsymbol{k}^{m} \\
& =-i \frac{e^{2} m_{D}^{2} \log (1 / e)}{4 \pi|\boldsymbol{p}|}\left(\frac{1}{\beta|\boldsymbol{p}|^{2}} c_{s}(|\boldsymbol{p}|)-\left(n_{s}(|\boldsymbol{p}|)-\frac{1}{2}\right)\left(c_{s}^{\prime}(|\boldsymbol{p}|)-\frac{c_{s}(|\boldsymbol{p}|)}{|\boldsymbol{p}|}\right)-\frac{1}{2 \beta} c_{s}^{\prime \prime}(|\boldsymbol{p}|)\right) \epsilon^{i l m} \hat{\boldsymbol{p}}^{l} \boldsymbol{k}^{m},
\end{aligned}
$$

up to leading $\log$ order.

The evaluation of the second integral in (5.40) is more complicated. Let us first look at the term $\chi_{s}(|\boldsymbol{p}+\boldsymbol{q}|) \widehat{\boldsymbol{p}+\boldsymbol{q}}^{i}$ in the integrand. Defining $\tilde{\chi}_{s}(x) \equiv \chi_{s}(x) / x$, and using the fact that the $\delta$ function in the integrand imposes $|\boldsymbol{p}+\boldsymbol{q}|=|\boldsymbol{p}|+s q^{0}$, we have

$$
\chi_{s}(|\boldsymbol{p}+\boldsymbol{q}|) \widehat{\boldsymbol{p}+\boldsymbol{q}^{i}}=\tilde{\chi}_{s}\left(|\boldsymbol{p}|+s q^{0}\right)\left(\boldsymbol{p}^{i}+\boldsymbol{q}^{i}\right) .
$$

Since what we need is the P-odd structure $\epsilon^{i l m} \hat{\boldsymbol{p}}^{l} \boldsymbol{k}^{m}$, it is clear that the first term proportional to $\boldsymbol{p}^{i}$ cannot possibly generate such a structure; therefore, it is sufficient to consider only the second piece proportional to $\boldsymbol{q}^{i}$,

$$
\chi_{s}(|\boldsymbol{p}+\boldsymbol{q}|) \widehat{\boldsymbol{p}+\boldsymbol{q}^{i}} \rightarrow \tilde{\chi}_{s}\left(|\boldsymbol{p}|+s q^{0}\right) \boldsymbol{q}^{i},
$$

in the integral of (5.40). On the other hand, because $\mathcal{K}_{s}^{(1)}(\boldsymbol{p}, \boldsymbol{k} ; Q)$ is a rotationally scalar function linear in $\boldsymbol{k}$, rotational invariance dictates that it can only have three possible structures,

$$
\mathcal{K}_{s}^{(1)}(\boldsymbol{p}, \boldsymbol{k} ; Q)=\left(a_{s}^{(1)} \hat{\boldsymbol{p}}^{l}+b_{s}^{(1)} \boldsymbol{q}^{l}+c_{s}^{(1)} \epsilon^{l m n} \hat{\boldsymbol{p}}^{m} \boldsymbol{q}^{n}\right) \boldsymbol{k}^{l},
$$

where $\left(a_{s}^{(1)}, b_{s}^{(1)}, c_{s}^{(1)}\right)$ are some coefficient functions that depend only on $\left(p^{0},|\boldsymbol{p}|, q^{0},|\boldsymbol{q}|, \boldsymbol{p} \cdot \boldsymbol{q}\right)$. Combining these two facts, and considering the rotational invariance of $\boldsymbol{q}$ integration, one can easily find that the only way to have the resulting P-odd structure $\epsilon^{i l m} \hat{\boldsymbol{p}}^{l} \boldsymbol{k}^{m}$ from the second integral in (5.40) is via the third term in (5.45); that is, we only need to find the part of $\mathcal{K}_{s}^{(1)}(\boldsymbol{p}, \boldsymbol{k} ; Q)$ that is proportional to $\epsilon^{l m n} \hat{\boldsymbol{p}}^{m} \boldsymbol{q}^{n} \boldsymbol{k}^{l}=\epsilon^{l m n} \hat{\boldsymbol{p}}^{l} \boldsymbol{q}^{m} \boldsymbol{k}^{n}$. This greatly simplifies our computation.

Since (5.44) is already linear in $Q$, for a leading log contribution we only need to expand $\mathcal{K}_{s}^{(1)}(\boldsymbol{p}, \boldsymbol{k} ; Q)$ up to linear order in $Q$, which is already saturated by the structure of interest $\epsilon^{l m n} \hat{\boldsymbol{p}}^{l} \boldsymbol{q}^{m} \boldsymbol{k}^{n}$. This in turn implies that one can neglect the $s q^{0}$ correction in (5.44) to have

$$
\chi_{s}(|\boldsymbol{p}+\boldsymbol{q}|) \widehat{\boldsymbol{p}+\boldsymbol{q}}^{i} \rightarrow \tilde{\chi}_{s}(|\boldsymbol{p}|) \boldsymbol{q}^{i}=\frac{\chi_{s}(|\boldsymbol{p}|)}{|\boldsymbol{p}|} \boldsymbol{q}^{i}
$$

in the integral of (5.40). Given the expressions for $\Sigma_{s}^{i}(\boldsymbol{p}, \boldsymbol{k})$ and $F_{s}(\boldsymbol{p} ; \boldsymbol{q}, \boldsymbol{k})$ in (5.5) and Appendix $\mathrm{C}$, as well as the photon spectral density given in (5.24),

$$
\begin{aligned}
\rho_{00}^{\mathrm{ph}} & =\rho_{L}(Q), \\
\rho_{i j}^{\mathrm{ph}}(Q) & =\rho_{T}(Q)\left(\delta_{i j}-\frac{\boldsymbol{q}^{i} \boldsymbol{q}^{j}}{|\boldsymbol{q}|^{2}}\right)+i \rho_{\mathrm{odd}}(Q) \epsilon^{i j l} \boldsymbol{q}_{l},
\end{aligned}
$$

it is straightforward to find, after some amount of algebra, that

$$
\begin{aligned}
\mathcal{K}_{s}^{(1)}(\boldsymbol{p}, \boldsymbol{k} ; Q)= & \left(n_{+}\left(s|\boldsymbol{p}|+q^{0}\right)+n_{B}\left(q^{0}\right)\right) \\
& \times\left(\frac{i s}{2|\boldsymbol{p}|^{2}}\left(\rho_{L}(Q)-\left(1+\frac{\left(q^{0}\right)^{2}}{|\boldsymbol{q}|^{2}}\right) \rho_{T}(Q)\right)\right. \\
& \left.+\frac{i}{|\boldsymbol{p}|} \rho_{\text {odd }}(Q)\right) \epsilon^{l m n} \hat{\boldsymbol{p}}^{l} \boldsymbol{q}^{m} \boldsymbol{k}^{n}
\end{aligned}
$$

up to linear order in $Q$, which will contribute to the leading $\log$ result of the integral in (5.40). Note that we have a non-negligible contribution from the P-odd part of the spectral density $\rho_{\text {odd }}(Q)$ : from its sum rule given in (5.25) one can easily see that this term engenders a leading $\log$ contribution to the integral. When combining $\mathcal{K}_{s}^{(1)}(\boldsymbol{p}, \boldsymbol{k} ; Q)$ with (5.46) in the integral of (5.40), one has to replace $\boldsymbol{q}^{m} \boldsymbol{q}^{i}$ with

$$
\begin{aligned}
\boldsymbol{q}^{m} \boldsymbol{q}^{i} & \rightarrow \frac{1}{2} \delta^{m i}\left|\boldsymbol{q}_{T}\right|^{2} \\
= & \frac{1}{2} \delta^{m i}\left(|\boldsymbol{q}|^{2}-(\hat{\boldsymbol{p}} \cdot \boldsymbol{q})^{2}\right)=\frac{1}{2}\left(|\boldsymbol{q}|^{2}-\left(q^{0}\right)^{2}\right),
\end{aligned}
$$

where $\boldsymbol{q}_{T}$ is the perpendicular component of $\boldsymbol{q}$ to $\boldsymbol{p}$, and where we use (5.18) in the last equality. This comes from the rotational invariance of the $\boldsymbol{q}$ integral around the $\hat{\boldsymbol{p}}$ axis. Collecting all these and following the same steps as in the leading log computation of $\chi_{s}(|\boldsymbol{p}|)$, we finally have the second integral of (5.40), given by, at leading log order, 


$$
\begin{aligned}
e^{2} \int & \frac{d^{4} Q}{(2 \pi)^{4}} \mathcal{K}_{s}^{(1)}(\boldsymbol{p}, \boldsymbol{k} ; Q) \chi_{s}(|\boldsymbol{p}+\boldsymbol{q}|) \widehat{\boldsymbol{p}+\boldsymbol{q}^{i}}(2 \pi) \delta\left(q^{0}+s|\boldsymbol{p}|-s|\boldsymbol{p}+\boldsymbol{q}|\right) \\
= & -\frac{e^{2}}{2} \frac{\chi_{s}(|\boldsymbol{p}|)}{|\boldsymbol{p}|} \int_{0}^{\infty} \frac{d|\boldsymbol{q} \| \boldsymbol{q}|}{(2 \pi)} \int_{-|\boldsymbol{q}|}^{|\boldsymbol{q}|} \frac{d q^{0}}{(2 \pi)}\left(1+\frac{s q^{0}}{|\boldsymbol{p}|}\right)\left(\frac{1}{\beta q^{0}}+s\left(n_{s}(|\boldsymbol{p}|)-\frac{1}{2}\right)\right) \\
& \times\left(\frac{i s}{2|\boldsymbol{p}|^{2}}\left(\rho_{L}(Q)-\left(1+\frac{\left(q^{0}\right)^{2}}{|\boldsymbol{q}|^{2}}\right) \rho_{T}(Q)\right)+\frac{i}{|\boldsymbol{p}|} \rho_{\text {odd }}(Q)\right)\left(|\boldsymbol{q}|^{2}-\left(q^{0}\right)^{2}\right) \epsilon^{i l m} \hat{\boldsymbol{p}}^{l} \boldsymbol{k}^{m} \\
= & -\frac{e^{2}}{2} \frac{\chi_{s}(|\boldsymbol{p}|)}{\beta|\boldsymbol{p}|} \int_{0}^{\infty} \frac{d|\boldsymbol{q} \| \boldsymbol{q}|}{(2 \pi)}\left(\frac{i s}{2|\boldsymbol{p}|^{2}}\left(|\boldsymbol{q}|^{2}\left(J_{0}^{L}-J_{0}^{T}-\frac{1}{|\boldsymbol{q}|^{2}} J_{1}^{T}\right)-J_{1}^{L}+J_{1}^{T}+\frac{1}{|\boldsymbol{q}|^{2}} J_{2}^{T}\right)+\frac{i}{|\boldsymbol{p}|}\left(-\frac{e^{2} \mu}{(2 \pi)^{2}|\boldsymbol{q}|^{2}}\right)\right) \epsilon^{i l m} \hat{\boldsymbol{p}}^{l} \boldsymbol{k}^{m} \\
= & i \frac{e^{2}}{4 \pi} \frac{\chi_{s}(|\boldsymbol{p}|)}{\beta|\boldsymbol{p}|^{2}} \log (1 / e) \frac{e^{2} \mu}{2 \pi^{2}} \epsilon^{i l m} \hat{\boldsymbol{p}}^{l} \boldsymbol{k}^{m},
\end{aligned}
$$

where we use the sum rules (5.30), (A26), and (A27). Interestingly, it turns out that the contributions from the P-even spectral densities $\rho_{L / T}$ cancel each other exactly. We do not have a good understanding of whether this must be the case by some symmetry reason, or whether it is just by accident. Therefore, the only contribution to the second integral in (5.40) at leading $\log$ order [that is, $\left.e^{4} \log (1 / e)\right]$ comes from the P-odd part of the soft (HTL) photon spectral density $\rho_{\text {odd }}(Q)$. Note that for this contribution, we have equal logarithmic contributions from both $e^{2} T \ll|\boldsymbol{q}| \ll m_{D}$ and $T \gg|\boldsymbol{q}| \gg m_{D}$, which add up together in the final result. $^{8}$

From the integral equation (5.40) with (5.42) and (5.50), we finally obtain the second order differential equation for $c_{s}(|\boldsymbol{p}|)$ as

$$
\begin{aligned}
\zeta_{\boldsymbol{p}, s}^{\mathrm{sf}} c_{s}(|\boldsymbol{p}|)= & -\frac{1}{2}+\frac{e^{4} \log (1 / e) \mu}{(4 \pi) 2 \pi^{2}} \frac{\chi_{s}(|\boldsymbol{p}|)}{\beta|\boldsymbol{p}|} \\
& -\frac{e^{2} m_{D}^{2} \log (1 / e)}{4 \pi}\left(\frac{1}{\beta|\boldsymbol{p}|^{2}} c_{s}(|\boldsymbol{p}|)-\left(n_{s}(|\boldsymbol{p}|)-\frac{1}{2}\right)\right. \\
& \left.\times\left(c_{s}^{\prime}(|\boldsymbol{p}|)-\frac{c_{s}(|\boldsymbol{p}|)}{|\boldsymbol{p}|}\right)-\frac{1}{2 \beta} c_{s}^{\prime \prime}(|\boldsymbol{p}|)\right),
\end{aligned}
$$

where the first line is an inhomogeneous sourceespecially the second term, which is in terms of $\chi_{s}(|\boldsymbol{p}|)$ and which should be obtained by solving the differential equation (5.32). We would need the expansion of $c_{s}(|\boldsymbol{p}|)$ up to first order in chemical potential $\mu$,

$$
c_{s}(|\boldsymbol{p}|)=c_{(0)}(|\boldsymbol{p}|)+s \mu c_{(1)}(|\boldsymbol{p}|)+\mathcal{O}\left(\mu^{2}\right),
$$

which can be found by solving the above differential equation order by order in $\mu$. We reemphasize that there are linear $\mu$ dependences coming from $\zeta_{p, s}^{\text {sf }}$ and $n_{s}(|\boldsymbol{p}|)$ in the above differential equation; to get a correct leading log answer, these should not be missed. After finding $\chi_{s}(|\boldsymbol{p}|)$ and $c_{s}(|\boldsymbol{p}|)$ from the differential equations given above, we compute our transport coefficient $\xi_{5}$ by (5.13) with $G_{(r a)}^{i j, \text { inch,P-odd }}(k)=i \omega \xi_{5} \epsilon^{i j l} \boldsymbol{k}^{l}$.

\section{Numerical evaluation}

As a first step in computing the explicit value of $\xi_{5}$, we solve numerically the equations for $\left(\chi_{s}(|\boldsymbol{p}|), c_{s}(|\boldsymbol{p}|)\right)$ in (5.32) and (5.51). In order to do so, we define $\Psi_{s}(|\boldsymbol{p}| / T) \equiv$ $\alpha m_{D}^{2} \log (1 / e) c_{s}(|\mathbf{p}|) / T$ and $\Phi_{s}(|\boldsymbol{p}| / T) \equiv \alpha m_{D}^{2} \log (1 / e) \times$ $\chi_{s}(|\mathbf{p}|) / T$ where $\alpha=e^{2} / 4 \pi$. Defining $y \equiv|\boldsymbol{p}| / T$, Eq. (5.51) can be rewritten as

$$
\begin{aligned}
& \Psi_{s}^{\prime \prime}(y)-\tanh (y / 2) \Psi_{s}^{\prime}(y)-\frac{\Psi_{s}(y)}{y}\left(\frac{3}{2} \operatorname{coth}(y / 2)-\tanh (y / 2)+\frac{2}{y}\right) \\
& =1-\frac{6 s \mu}{\pi^{2} T}\left(\frac{s \Phi_{s}(y)}{y}+\frac{\pi^{2}}{8} \frac{\Psi_{s}(y)}{y}\right)+s \frac{\mu}{T} \frac{\operatorname{sech}^{2}(y / 2)}{2}\left(\frac{\Psi_{s}(y)}{y}-\Psi_{s}^{\prime}(y)\right) .
\end{aligned}
$$

In addition, we have the equation for the even vertex, $\chi_{s}(|\boldsymbol{p}|)$ in (5.32),

$$
\begin{aligned}
& \Phi_{s}^{\prime \prime}(y)+\left(\frac{2}{y}-\tanh (y / 2)\right) \Phi_{s}^{\prime}(y)-\left(\frac{2}{y^{2}}+\frac{3 \operatorname{coth}(y / 2)}{2 y}\right) \Phi_{s}(y) \\
& =-2 s-s \frac{3 \mu}{4 T} \frac{\Phi_{s}(y)}{y}-s \frac{\mu}{2 T} \operatorname{sech}^{2}(y / 2) \Phi_{s}^{\prime}(y) .
\end{aligned}
$$

\footnotetext{
${ }^{8}$ Strictly speaking, QED does not possess the ultrasoft magnetic cutoff $\sim e^{2} T$. Since we have in mind the generalization to nonAbelian QCD as discussed in Sec. V, we simply assume this at this point.
} 
In the above, we expand the $\mu$ dependence from $\zeta_{\boldsymbol{p}, s}^{\text {sf }}$ and $n_{s}(|\boldsymbol{p}|)$ up to linear order in $\mu$. We then expand the solution to first order in $\mu$ as

$$
\begin{aligned}
& \Psi_{s}=\Psi_{A}+s \frac{\mu}{T} \Psi_{B}+\mathcal{O}\left(\mu^{2}\right), \\
& \Phi_{s}=s \Phi_{A}+\frac{\mu}{T} \Phi_{B}+\mathcal{O}\left(\mu^{2}\right),
\end{aligned}
$$

from which we have a coupled set of differential equations

$$
\begin{aligned}
\Psi_{A}^{\prime \prime}(y)-\tanh (y / 2) \Psi_{A}^{\prime}(y)-\frac{\Psi_{A}(y)}{y}\left(\frac{3}{2} \operatorname{coth}(y / 2)-\tanh (y / 2)+\frac{2}{y}\right)= & 1, \\
\Psi_{B}^{\prime \prime}(y)-\tanh (y / 2) \Psi_{B}^{\prime}(y)-\frac{\Psi_{B}(y)}{y}\left(\frac{3}{2} \operatorname{coth}(y / 2)-\tanh (y / 2)+\frac{2}{y}\right)= & -\frac{6}{\pi^{2}} \frac{\Phi_{A}(y)}{y}-\frac{3}{4} \frac{\Psi_{A}(y)}{y} \\
& +\frac{\operatorname{sech}^{2}(y / 2)}{2}\left(\frac{\Psi_{A}(y)}{y}-\Psi_{A}^{\prime}(y)\right), \\
\Phi_{A}^{\prime \prime}(y)+\left(\frac{2}{y}-\tanh (y / 2)\right) \Phi_{A}^{\prime}(y)-\left(\frac{2}{y^{2}}+\frac{3 \operatorname{coth}(y / 2)}{2 y}\right) \Phi_{A}= & -2, \\
\Phi_{B}^{\prime \prime}(y)+\left(\frac{2}{y}-\tanh (y / 2)\right) \Phi_{B}^{\prime}(y)-\left(\frac{2}{y^{2}}+\frac{3 \operatorname{coth}(y / 2)}{2 y}\right) \Phi_{B}(y)= & -\frac{3}{4} \frac{\Phi_{A}(y)}{y}-\frac{1}{2} \operatorname{sech}^{2}(y / 2) \Phi_{A}^{\prime}(y) .
\end{aligned}
$$

We solve the above equations by an iterative method with vanishing boundary conditions at the IR $(y=0)$ and the UV $(y=\infty)$. The last step is then to obtain the expression for the transport coefficient $\xi_{5}$ as an integral of the above quantities. After computing the sum over $s= \pm$ and performing angular integrations in (5.13), we obtain the result for the retarded propagator as an integral of $\Psi_{A / B}(y)$ and $\Phi_{A / B}(y)$,

$$
\begin{aligned}
G_{(r a)}^{i j, \text { Pinch }}(k)= & \frac{2 i \omega \mu \epsilon^{i j l} \boldsymbol{k}_{l}}{T \pi e^{4} \log (1 / e)} \int_{0}^{\infty} y d y\left(\left(\Psi_{B}(y)-\frac{\Phi_{B}(y)}{2}\right)\right. \\
& \times \frac{1}{\cosh ^{2}(y / 2)}+\left(\Psi_{A}(y)-\frac{\Phi_{A}(y)}{2}\right) \\
& \left.\times \frac{\tanh ^{2}(y / 2)}{\cosh ^{2}(y / 2)}\right) .
\end{aligned}
$$

From the identification $G_{(r a)}^{i j \text { Pinch,P-odd }}(k)=i \omega \xi_{5} \epsilon^{i j l} \boldsymbol{k}^{l}$, we find

$$
\xi_{5}=-\frac{3.006}{e^{4} \log (1 / e)} \frac{\mu}{T}
$$

\section{DISCUSSION}

It is easy to generalize the above to the case of $N_{F}$ species of Dirac fermions in an $S U\left(N_{c}\right)$ gauge theory [though the current and magnetic field are still with respect to the global $U(1)$ flavor symmetry]. The chemical potential appearing in the integral equation is simply the axial chemical potential $\mu_{A}$. The Debye mass and asymptotic thermal mass are changed to

$$
m_{D}^{2}=\frac{g^{2} T^{2}}{6}\left(2 N_{c}+N_{F}\right), \quad m_{f}^{2}=\frac{g^{2} T^{2}}{4} \frac{N_{c}^{2}-1}{2 N_{c}} .
$$

The soft-fermion contribution to the hard photon damping rate is given by

$$
\zeta_{\boldsymbol{p}, s}^{\mathrm{sf}}=\frac{g^{2}}{4 \pi} \frac{N_{c}^{2}-1}{2 N_{c}} \frac{m_{f}^{2} \log (1 / g)}{|\boldsymbol{p}|}\left(n_{B}(|\boldsymbol{p}|)+n_{-s}(0)\right) .
$$

In the integral equations (5.32) and (5.51), the $e^{2} \log (1 / e)$ in the kernel part should be replaced by

$$
g^{2} \log (1 / g) \frac{N_{c}^{2}-1}{2 N_{c}}
$$

In the second source term in the first line of (5.51), which is proportional to $e^{4} \log (1 / e)$, this $e^{4} \log (1 / e)$ should be replaced by

$$
g^{4} \log (1 / g) N_{F} \frac{N_{c}^{2}-1}{2 N_{c}}
$$

This is because one factor of $e^{2}$ coming from the spectral density $\rho_{\text {odd }}(Q)$ is replaced by $g^{2} N_{F}$, while the other $e^{2}$ coming from fermion-gluon couplings in the kernel is 
replaced by $g^{2}\left(N_{c}^{2}-1\right) /\left(2 N_{c}\right)$. Finally, the expression for $G_{(\mathrm{ra})}^{i j \text { Pinch }}(k)$ from (5.13), or, equivalently, our $\xi_{5}$ from $\chi_{s}(|\boldsymbol{p}|)$ and $c_{s}(|\boldsymbol{p}|)$, must be multiplied by the fermion degeneracy

$$
2 N_{c} \times \sum_{F} Q_{F}^{2}
$$

where $Q_{F}$ are charges of $F$ flavors in units of $e$ [for $(u, d)$ quarks, it is $Q_{u}=2 / 3$ and $Q_{d}=-1 / 3$ ]. For two-flavor massless QCD $\left(N_{c}=3\right)$ with $Q_{u}=2 / 3$ and $Q_{d}=-1 / 3$, the result for $\xi_{5}$ then becomes

$$
\xi_{5}^{\mathrm{QCD}}=-\frac{2.003}{g^{4} \log (1 / g)} \frac{\mu}{T} .
$$

As mentioned at the end of Sec. II, the color conductivity $\sigma_{c}$ for non-Abelian gauge theory that appears in the lowenergy effective theory at the scale $Q \lesssim g^{2} T$,

$$
\boldsymbol{J}^{a}=\sigma_{c} \boldsymbol{E}^{a}+\boldsymbol{\xi}^{a},
$$

where $a$ denotes adjoint color charge and $\boldsymbol{\xi}^{a}$ is the thermal noise via fluctuation-dissipation relation to $\sigma_{c}$, is governed by scatterings with ultrasoft transverse thermal gluons of momenta $Q \ll g T$ with the rate $\sim g^{2} \log (1 / g)$; this leads to $\sigma_{c} \sim g^{2} \cdot 1 /\left(g^{2} \log (1 / g)\right) \sim 1 / \log (1 / g) .{ }^{9}$ To obtain a correct leading $\log$ result for this, one needs a similar diagrammatic resummation with essentially the same technique as in our computation, except that charge carriers include gluons as well as quarks, and there is no longer a precise cancellation of the $g^{2} \log (1 / g)$ terms in the integral equation; this is due to the absence of the U(1) Ward identity (replaced by a non-Abelian version of the SlavnovTaylor identity) that ensures the cancellation of these terms [59]. The diagrammatic resummation (done in Ref. [69]) for this is, therefore, somewhat simpler than that for the electric conductivity, since one does not need to go to the next order of $g^{4} \log (1 / g)$. In fact, one has an algebraic equation to solve, rather than a differential equation. The same resummation can also be achieved in the language of Bodecker's approach [64] as well as in kinetic theory [65]. In the presence of the axial charge, $\mu_{A}$, which breaks $C P$ symmetry, Ref. [70] recently obtained, via Bodecker's approach, a $C P$-odd contribution to the color current

$$
\boldsymbol{J}^{a}=\sigma_{c} \boldsymbol{E}^{a}+\boldsymbol{\xi}^{a}+\frac{\mu_{A} N_{F} g^{2}}{4 \pi^{2}} \boldsymbol{B}^{a},
$$

which is a colored analogue of the chiral magnetic effect consistent with the $U(1)_{A} S U(3)_{c}^{2}$ triangle anomaly. Since the triangle anomaly is topological, this contribution should be saturated at one loop diagrammatically, without the need

\footnotetext{
${ }^{9}$ Note that we now put an extra $g^{2}$ in the definition of color conductivities to follow the convention in the literature.
}

for the resummation of ladder diagrams. From our computation in the text, the quantity that needs a ladder resummation, and which is sensitive to the same ultrasoft scale dynamics of the $g^{2} \log (1 / g)$ rate to which the color conductivity is also sensitive, appears when one goes to the next order in the derivative,

$$
\boldsymbol{J}^{a}=\sigma_{c} \boldsymbol{E}^{a}+\boldsymbol{\xi}^{a}+\frac{\mu_{A} N_{F} g^{2}}{4 \pi^{2}} \boldsymbol{B}^{a}+\xi_{5}^{c} \frac{d \boldsymbol{B}^{a}}{d t} .
$$

It is clear that $\xi_{5}^{c}$, a colored analogue of our $\xi_{5}$, will be of order

$$
\xi_{5}^{c} \sim g^{2} \cdot 1 /\left(g^{2} \log (1 / g)\right)\left(\mu_{A} / T\right) \sim(1 / \log (1 / g))\left(\mu_{A} / T\right)
$$

due to the absence of the precise cancellation of the $g^{2} \log (1 / g)$ terms in the integral equations. The computation of $\xi_{5}^{c}$ at leading log order is doable, following the same steps that we present in our work and keeping only the $g^{2} \log (1 / g)$ terms in the integral equations (note that it receives contributions only from quarks, not from gluons). The leading order fermion damping rate from soft gluon scatterings is

$$
\zeta_{\boldsymbol{p}, s}^{\mathrm{sp}}=\frac{N_{c}^{2}-1}{2 N_{c}} \frac{g^{2} \log (1 / g) T}{2 \pi}
$$

and the solution of the integral equations that become algebraic is

$$
\begin{aligned}
& \chi_{s}(|\boldsymbol{p}|)=\frac{s}{\zeta_{\boldsymbol{p}, s}^{\mathrm{sp}}+\frac{1}{2 N_{c}} \frac{g^{2} \log (1 / g) T}{2 \pi}}=\frac{4 \pi s}{N_{c} g^{2} \log (1 / g) T}, \\
& c_{s}(|\boldsymbol{p}|)=-\frac{s}{2} \chi_{s}(|\boldsymbol{p}|) .
\end{aligned}
$$

This gives our result for $\xi_{5}^{c}$,

$$
\begin{aligned}
\xi_{5}^{c}= & -\left.\frac{g^{2} N_{F}}{3} \int \frac{d^{3} \boldsymbol{p}}{(2 \pi)^{3}} \sum_{s= \pm} \frac{d n_{+}\left(p^{0}\right)}{d p^{0}}\right|_{p^{0}=s|\boldsymbol{p}|} \frac{1}{|\boldsymbol{p}|} \\
& \times\left(s c_{s}(|\boldsymbol{p}|)-\frac{1}{2} \chi_{s}(|\boldsymbol{p}|)\right) \\
= & -\frac{2}{3 \pi} \frac{N_{F}}{N_{c}} \frac{1}{\log (1 / g)} \frac{\mu_{A}}{T} .
\end{aligned}
$$

The same resummation should also be achievable via Bodecker's approach, presented in Ref. [70], by going to the next order in time derivatives.

It is of the utmost importance to implement the correct value of the chiral magnetic current in the presence of a time-varying magnetic field in any realistic simulation of the chiral magnetic effect (or any other anomaly-induced transport phenomena) in heavy-ion collisions. In the weak 
coupling picture, our results should be an important step toward taking into account the time-varying nature of the magnetic field in heavy-ion collisions, and they will be instrumental in the quantitative studies of the chiral anomaly-induced phenomena in the experiments at the RHIC and the LHC.

\section{ACKNOWLEDGMENTS}

A. J. would like to thank the Nuclear Theory Group at UIC for hospitality during his visit and Francisco Pena for useful comments. H. U. Y. thanks Sangyong Jeon, Dima Kharzeev, Kiminad Mamo, Rob Pisarski, Daisuke Satow, and Misha Stephanov for discussions. We thank Kristan Jensen for helpful correspondence on hydrostatic constraint analysis. A. J. has been supported by a FPU fellowship Grant No. AP2010-5686, Plan Nacional de Altas Energias Grant No. FPA2009-07890, Consolider Ingenio 2010 CPAN Grant No. CSD200-00042, and Severo Ochoa Grant No. SEV-2012-0249.

\section{APPENDIX A: SUM RULES FOR THE P-ODD PARAT OF HTL PHOTON SPECTRAL DENSITY}

Let us start from the thermal relation

$$
G_{\mu \nu}^{r r}(q)=\left(\frac{1}{2}+n_{B}\left(q^{0}\right)\right)\left(G_{\mu \nu}^{r a}(q)-G_{\mu \nu}^{a r}(q)\right)
$$

where $G_{\mu \nu}^{a b}(x) \equiv\left\langle A_{\mu}^{a}(x) A_{\nu}^{b}(0)\right\rangle_{\mathrm{SK}}(a, b=r, a)$ are correlation functions in the Schwinger-Keldysh path integral, and

$$
G_{\mu \nu}^{a r}(x)=\left\langle A_{\mu}^{a}(x) A_{\nu}^{r}(0)\right\rangle_{\mathrm{SK}}=G_{\nu \mu}^{r a}(-x),
$$

where we use the translational invariance of the system. Since what we encounter in writing down our integral equations in the main text is the combination $\left[G_{\mu \nu}^{r a}(q)-\right.$ $\left.G_{\mu \nu}^{a r}(q)\right]$, let us naturally define the photon spectral density (including possible P-odd contributions in general)

$$
\rho_{\mu \nu}^{\mathrm{ph}}(q) \equiv G_{\mu \nu}^{r a}(q)-G_{\mu \nu}^{a r}(q) .
$$

We will show that $\rho_{\mu \nu}^{\mathrm{ph}}(q)$ is in general a Hermitian matrix in terms of $\mu \nu$ indices. For diagonal components that come from the usual P-even contributions, $\rho_{\mu \nu}^{\mathrm{ph}, \mathrm{P}-\mathrm{even}}(q)$ is therefore real. For the P-odd contribution that turns out to be antisymmetric in spatial $i j$ indices (there is no P-odd contribution to the timelike component, at least up to linear order in $\mu$ ), we thus have $\rho_{i j}^{\mathrm{ph}, \text { P-even }}(q)$ as purely imaginary.

To show that $\rho_{\mu \nu}^{\mathrm{ph}}(q)$ is a Hermitian matrix, recall that the usual retarded propagator is defined as

$$
G_{\mu \nu}^{R}(x)=-i \theta\left(x^{0}\right)\left\langle\left[A_{\mu}(x), A_{\nu}(0)\right]\right\rangle=-i G_{\mu \nu}^{r a}(x) .
$$

It is not difficult to show, using the Hermiticity of $A_{\mu}$, that $G_{\mu \nu}^{R}(x)$ is real valued; this is what it should be since the retarded propagator gives the response of the system in real time, which must be real valued. Therefore, in Fourier space, one has

$$
G_{\mu \nu}^{R}(-q)=\left(G_{\mu \nu}^{R}(q)\right)^{*},
$$

which in turn gives

$$
G_{\mu \nu}^{r a}(-q)=-\left(G_{\mu \nu}^{r a}(q)\right)^{*} .
$$

On the other hand, from (A2) we have

$$
G_{\mu \nu}^{a r}(q)=G_{\nu \mu}^{r a}(-q)=-\left(G_{\nu \mu}^{r a}(q)\right)^{*},
$$

where we use (A6) in the last equality. Therefore

$$
\rho_{\mu \nu}^{\mathrm{ph}}(q)=G_{\mu \nu}^{r a}(q)-G_{\mu \nu}^{a r}(q)=G_{\mu \nu}^{r a}(q)+\left(G_{\nu \mu}^{r a}(q)\right)^{*},
$$

which proves that $\rho_{\mu \nu}^{\mathrm{ph}}(q)$ is indeed a Hermitian matrix.

The P-odd part of the retarded current-current correlation functions, which is the retarded photon self-energy, has been recently computed in the literature in the HTL limit $[71,72]$. We will work in the Coulomb gauge where $G_{0 i}^{r a}=$ $0(i=1,2,3)$. The P-odd contribution appears only in the spatial transverse part of the correlation functions; therefore, we will discuss only the spatial transverse part of current correlation functions in the following. In matrix notation, the spatial part of the HTL resummed photon propagator is

$$
\left(G^{r a}(q)\right)^{-1}=\left(G_{(0)}^{r a}(q)\right)^{-1}-i \Sigma^{R}(q),
$$

where $G_{(0)}^{r a}(q)$ is the bare propagator given by

$$
G_{(0) i j}^{r a}(q)=\frac{-i P_{i j}^{T}(\boldsymbol{q})}{-\left(q^{0}+i \epsilon\right)^{2}+|\boldsymbol{q}|^{2}} .
$$

The HTL self-energy $\Sigma^{R}(q)$ including the P-odd contribution is given by

$$
\Sigma_{i j}^{R}(q)=\Pi_{T}(q) P_{i j}^{T}(\boldsymbol{q})+i \Pi_{\mathrm{odd}}(q) \epsilon^{i j l} \boldsymbol{q}^{l},
$$

where 


$$
\begin{aligned}
\Pi_{T}(q)= & -\frac{m_{D}^{2}}{2}\left(\frac{\left(q^{0}\right)^{2}}{|\boldsymbol{q}|^{2}}+\left(\frac{\left(q^{0}\right)^{2}}{|\boldsymbol{q}|^{2}}-1\right)\right. \\
& \left.\times \frac{q^{0}}{2|\boldsymbol{q}|} \log \left(\frac{q^{0}-|\boldsymbol{q}|+i \epsilon}{q^{0}+|\boldsymbol{q}|+i \epsilon}\right)\right), \\
\Pi_{\text {odd }}(q)= & -\frac{e^{2} \mu}{4 \pi^{2}}\left(1-\frac{\left(q^{0}\right)^{2}}{|\boldsymbol{q}|^{2}}-\left(\frac{\left(q^{0}\right)^{2}}{|\boldsymbol{q}|^{2}}-1\right) \frac{q^{0}}{2|\boldsymbol{q}|}\right. \\
& \left.\times \log \left(\frac{q^{0}-|\boldsymbol{q}|+i \epsilon}{q^{0}+|\boldsymbol{q}|+i \epsilon}\right)\right),
\end{aligned}
$$

with $m_{D}^{2}=e^{2}\left(T^{2} / 6+\mu^{2} /\left(2 \pi^{2}\right)\right)$. From this, while keeping terms only up to linear order in $\mu$, we have

$$
\begin{aligned}
G_{i j}^{r a}(q)= & \frac{-i P_{i j}^{T}(\boldsymbol{q})}{-\left(q^{0}\right)^{2}+|\boldsymbol{q}|^{2}-\Pi_{T}(q)} \\
& +\frac{\Pi_{\mathrm{odd}}(q)}{\left(-\left(q^{0}\right)^{2}+|\boldsymbol{q}|^{2}-\Pi_{T}(q)\right)^{2}} \epsilon^{i j l} \boldsymbol{q}^{l},
\end{aligned}
$$

where the first term is the usual P-even HTL photon propagator, and the second term is the new P-odd contribution. The HTL photon spectral density $\rho_{i j}^{\mathrm{ph}}(q)$ is then given by

$$
\rho_{i j}^{\mathrm{ph}}(q)=\rho_{T}(q) P_{i j}^{T}(\boldsymbol{q})+i \rho_{\mathrm{odd}}(q) \epsilon^{i j l} \boldsymbol{q}^{l},
$$

with

$$
\begin{aligned}
& \rho_{T}(q)=2 \operatorname{Im}\left(\frac{1}{-\left(q^{0}\right)^{2}+|\boldsymbol{q}|^{2}-\Pi_{T}(q)}\right) \\
& \rho_{\text {odd }}(q)=2 \operatorname{Im}\left(\frac{\Pi_{\text {odd }}(q)}{\left(-\left(q^{0}\right)^{2}+|\boldsymbol{q}|^{2}-\Pi_{T}(q)\right)^{2}}\right) .
\end{aligned}
$$

It is easy to see that $\rho_{\text {odd }}(q)$ is an odd function in $q^{0}$, and what we need in the main text is the value of the integral

$$
J_{n}^{\text {odd }} \equiv \int_{-|q|}^{|q|} \frac{d q^{0}}{(2 \pi)}\left(q^{0}\right)^{2 n-1} \rho_{\text {odd }}(q), \quad n=0,1,2, \ldots
$$

One can compute them using the well-known sum-rule techniques exploring analytic properties of the function $\Delta_{\text {odd }}(q)$ defined by

$$
\Delta_{\text {odd }}(q) \equiv \frac{\Pi_{\text {odd }}(q)}{\left(-\left(q^{0}\right)^{2}+|\boldsymbol{q}|^{2}-\Pi_{T}(q)\right)^{2}} .
$$

We briefly sketch the procedure and present the results in two different regimes, $|\boldsymbol{q}| \ll m_{D}$ and $|\boldsymbol{q}| \gg m_{D}$.

The starting point is the fact that $\Delta_{\text {odd }}(q)$ in the complex $q^{0}$ plane is analytic in the upper half-plane due to the causal nature of a retarded function. Thus, the integral

$$
\int_{-\infty}^{\infty} \frac{d q^{0}}{2 \pi} \frac{1}{q^{0}-\omega+i \epsilon} \Delta_{\text {odd }}(q)=0
$$

vanishes for any real number $\omega$ by closing the contour with the upper hemicircle at infinity [and with $\Delta_{\text {odd }}(q) \rightarrow 0$ sufficiently fast as $\left.\left|q^{0}\right| \rightarrow \infty\right]$. From $1 /\left(q^{0}-\omega+i \epsilon\right)=$ $\mathcal{P} 1 /\left(q^{0}-\omega\right)-i \pi \delta\left(q^{0}-\omega\right)$, where $\mathcal{P}$ is the principal integration, we have

$$
\mathcal{P} \int_{-\infty}^{\infty} \frac{d q^{0}}{2 \pi} \frac{1}{q^{0}-\omega} \Delta_{\text {odd }}(q)-\frac{i}{2} \Delta_{\text {odd }}(\omega,|\boldsymbol{q}|)=0 .
$$

Considering the imaginary part of the above, we obtain one of the Kramers-Kronig dispersion relations for a retarded function (the real part gives the other dispersion relation),

$$
\mathcal{P} \int_{-\infty}^{\infty} \frac{d q^{0}}{2 \pi} \frac{1}{q^{0}-\omega} \rho_{\text {odd }}(q)=\operatorname{Re}\left[\Delta_{\text {odd }}(\omega,|\boldsymbol{q}|)\right] .
$$

Setting $\omega=0$ and using $\Delta_{\text {odd }}(0,|\boldsymbol{q}|)=-e^{2} \mu /\left(4 \pi^{2}|\boldsymbol{q}|^{4}\right)$, one obtains a sum rule

$$
\int_{-\infty}^{\infty} \frac{d q^{0}}{2 \pi} \frac{1}{q^{0}} \rho_{\text {odd }}(q)=-\frac{e^{2} \mu}{4 \pi^{2}|\boldsymbol{q}|^{4}} .
$$

Other sum rules are obtained from (A20) by expanding both sides in $\omega \rightarrow \infty$. The left-hand side becomes

$$
-\sum_{n=0}^{\infty} \frac{1}{\omega^{n+1}} \int_{-\infty}^{\infty} \frac{d q^{0}}{2 \pi}\left(q^{0}\right)^{n} \rho_{\text {odd }}(q),
$$

while the right-hand side when expanded in large $\omega$ is $-e^{2} \mu /\left(12 \pi^{2} \omega^{4}\right)+\mathcal{O}\left(1 / \omega^{6}\right)$; this gives two other sum rules,

$$
\begin{gathered}
\int_{-\infty}^{\infty} \frac{d q^{0}}{2 \pi} q^{0} \rho_{\text {odd }}(q)=0, \\
\int_{-\infty}^{\infty} \frac{d q^{0}}{2 \pi}\left(q^{0}\right)^{3} \rho_{\text {odd }}(q)=\frac{e^{2} \mu}{12 \pi^{2}} .
\end{gathered}
$$

The sum rules (A21) and (A23) are not precisely $J_{n}^{\text {odd }}$ as defined in (A16), because the integration range for $J_{n}^{\text {odd }}$ is $[-|\boldsymbol{q}|,+|\boldsymbol{q}|]$, not $[-\infty,+\infty]$. The imaginary part of $\Delta(q)$ [that is, $\rho_{\text {odd }}(q)$ ] consists of two distinct parts: one part coming from a branch cut just below the real line along the interval $q^{0} \in[-|\boldsymbol{q}|,+|\boldsymbol{q}|]$ from the logarithms in (A12) (originated from the Landau damping), and the other part from the two poles $\pm \omega_{0}$ satisfying $-\left(\omega_{0}\right)^{2}+|\boldsymbol{q}|^{2}-$ $\Pi_{T}\left(\omega_{0},|\boldsymbol{q}|\right)=0$ corresponding to the timelike transverse photons in the medium. The former has a continuous support in the interval $q^{0} \in[-|\boldsymbol{q}|,+|\boldsymbol{q}|]$, and hence contributes to $J_{n}^{\text {odd }}$, while the latter's pole contributions sit outside the interval, $\omega_{0}>|\boldsymbol{q}|$, and so do not contribute to $J_{n}^{\text {odd }}$. Therefore, the only difference between the sum rule 
values in (A21), (A23) and the $J_{n}^{\text {odd }}$ is simply the latter's pole contributions, which we can compute.

Near the pole location $q^{0} \approx \omega_{0}-i \epsilon$, we have the expansion

$$
\begin{aligned}
\left(q^{0}\right)^{2 n-1} 2 \Delta_{\text {odd }}(q) \approx & \frac{A}{\left(q^{0}-\omega+i \epsilon\right)^{2}}+\frac{B}{q^{0}-\omega_{0}+i \epsilon} \\
& +\{\text { regular }\}
\end{aligned}
$$

where

$$
\begin{aligned}
B= & \frac{2\left(\omega_{0}\right)^{2 n-1} \Pi_{\mathrm{odd}}\left(\omega_{0}\right)}{\left(2 \omega_{0}+\Pi_{T}^{\prime}\left(\omega_{0}\right)\right)^{2}} \\
& \times\left(\frac{\Pi_{\text {odd }}^{\prime}\left(\omega_{0}\right)}{\Pi_{\text {odd }}\left(\omega_{0}\right)}+\frac{(2 n-1)}{\omega_{0}}-\frac{2+\Pi_{T}^{\prime \prime}\left(\omega_{0}\right)}{2 \omega_{0}+\Pi_{T}^{\prime}\left(\omega_{0}\right)}\right),
\end{aligned}
$$

with $\Pi_{T}^{\prime}\left(q^{0}\right) \equiv d \Pi_{T}\left(q^{0},|\boldsymbol{q}|\right) / d q^{0}$, etc. The first double pole does not contribute to the imaginary part in the $\epsilon \rightarrow 0$ limit, while the second part contributes to $\left(q^{0}\right)^{2 n-1} \rho_{\text {odd }}(q)$ as $-\pi B \delta\left(q^{0}-\omega_{0}\right) \times 2=-2 \pi B \delta\left(q^{0}-\omega_{0}\right)$ (the factor of 2 comes from having two poles $\left.\pm \omega_{0}\right)$. This leads to the difference between the sum rules values in (A21), (A23) and the $J_{n}^{\text {odd }}$ as given by $-B$; that is, $J_{n}^{\text {odd }}$ is obtained by adding $B$ to the sum rule values in (A21), (A23).

In the case $|\boldsymbol{q}| \ll m_{D}$, the pole location is $\omega_{0} \approx \sqrt{1 / 3} m_{D}\left(1+(9 / 5)|\boldsymbol{q}|^{2} / m_{D}^{2}\right)+\cdots$, and an explicit computation of $B$ gives the values of $J_{n}^{\text {odd }}$ in this regime as

$$
\begin{aligned}
& J_{0}^{\text {odd }} \approx-\frac{e^{2} \mu}{4 \pi^{2}|\boldsymbol{q}|^{4}}+\frac{3 e^{2} \mu}{4 \pi^{2} m_{D}^{4}}+\mathcal{O}\left(|\boldsymbol{q}|^{2} / m_{D}^{6}\right), \\
& J_{1}^{\text {odd }} \approx-\frac{3 e^{2} \mu|\boldsymbol{q}|^{2}}{5 \pi^{2} m_{D}^{4}}+\mathcal{O}\left(|\boldsymbol{q}|^{4} / m_{D}^{6}\right), \\
& J_{2}^{\text {odd }} \approx-\frac{33 e^{2} \mu|\boldsymbol{q}|^{4}}{700 \pi^{2} m_{D}^{4}}+\mathcal{O}\left(|\boldsymbol{q}|^{6} / m_{D}^{6}\right) .
\end{aligned}
$$

On the other hand, in the regime $|\boldsymbol{q}| \gg m_{D}$, the poles are located in $\omega_{0} \approx|\boldsymbol{q}|+(1 / 4) m_{D}^{2} /|\boldsymbol{q}|+\cdots$, and we have the results in this regime $|\boldsymbol{q}| \gg m_{D}$ as

$$
\begin{aligned}
& J_{0}^{\text {odd }} \approx \frac{e^{2} \mu}{8 \pi^{2}|\boldsymbol{q}|^{4}}\left(1+\log \left(\frac{m_{D}^{2}}{8|\boldsymbol{q}|^{2}}\right)\right), \\
& J_{1}^{\text {odd }} \approx \frac{e^{2} \mu}{8 \pi^{2}|\boldsymbol{q}|^{2}}\left(3+\log \left(\frac{m_{D}^{2}}{8|\boldsymbol{q}|^{2}}\right)\right), \\
& J_{2}^{\text {odd }} \approx \frac{e^{2} \mu}{8 \pi^{2}}\left(\frac{11}{3}+\log \left(\frac{m_{D}^{2}}{8|\boldsymbol{q}|^{2}}\right)\right) .
\end{aligned}
$$

The above results (A26) and (A27) will be used in the main text in Sec. III.
APPENDIX B: HARD FERMION DAMPING RATE

In this Appendix, we compute the damping rate of hard fermions including the possible dependence on the chemical potential $\mu$. Our primary objective is twofold: First, we would like to confirm that the integral we have in (5.15) is indeed precisely equal to the damping rate induced by soft photon scatterings at full order in $e$ and $\mu$, which was instrumental in rewriting the integral equation to take the form (5.16) that contains only $e^{4} \log (1 / e)$ terms. Our second objective is to find a linear $s \mu$ dependence in the $\zeta_{p, s}^{\text {sf }}$, that is, in the damping rate induced by soft fermion scatterings (or, equivalently, fermion conversion-to-photon processes). This $s \mu$ dependence in $\zeta_{\boldsymbol{p}, s}^{\mathrm{sf}}$ is important for finding the correct $\mu$ dependence in the solution of differential equation (5.32) for $\chi_{s}(|\boldsymbol{p}|)$, which is crucial to obtain the correct result for $\xi_{5}$ as well as the $\mu^{2}$ correction to the usual electric conductivity.

Let us start with the self-energy resummed $r a$ propagator (which is equal to $i$ times the retarded propagator)

$$
\begin{aligned}
S^{r a}(p) & =S_{(0)}^{r a}(p)+S_{(0)}^{r a}(p) \Sigma^{r a}(p) S_{(0)}^{r a}(p)+\cdots \\
& =S_{(0)}^{r a}(p) \frac{1}{1-\Sigma^{r a}(p) S_{(0)}^{r a}(p)}
\end{aligned}
$$

which gives

$$
\left(S^{r a}(p)\right)^{-1}=\left(S_{(0)}^{r a}(p)\right)^{-1}-\Sigma^{r a}(p),
$$

where

$$
S_{(0)}^{r a}(p)=\sum_{s} \frac{i}{p^{0}-s|\boldsymbol{p}|+i \epsilon} \mathcal{P}_{s}(\boldsymbol{p})
$$

is the bare $r a$ propagator, and the self-energy $\Sigma^{r a}(p)$ (which is a $2 \times 2$ matrix in the spinor space) at naive lowest order in the coupling is given by two Feynman diagrams in Fig. 8 in real-time formalism with the expression

$$
\begin{aligned}
\Sigma^{r a}(p)= & (i e)^{2} \sigma^{\beta} \int \frac{d^{4} Q}{(2 \pi)^{4}}\left[G_{\alpha \beta}^{r r}(Q) S^{r a}(p+Q)\right. \\
& \left.+G_{\alpha \beta}^{a r}(Q) S^{r r}(p+Q)\right] \sigma^{\alpha},
\end{aligned}
$$

with the photon propagators $G_{\alpha \beta}^{a b}(Q)(a, b=r, a)$ (see Appendix A for our notational conventions). In the above expression, we have not specified whether the propagators appearing in the loop are bare or HTL-resummed ones, because, depending on the situations, we can consider different approximations for them to obtain the right leading order quantities. For example, if the external momentum $p$ is soft and one is interested in the HTL approximation, it is enough to consider hard loop momentum $Q$, and both propagators in the loop are bare ones. On the other hand, in the case of damping rate with a hard momentum $p$, which 

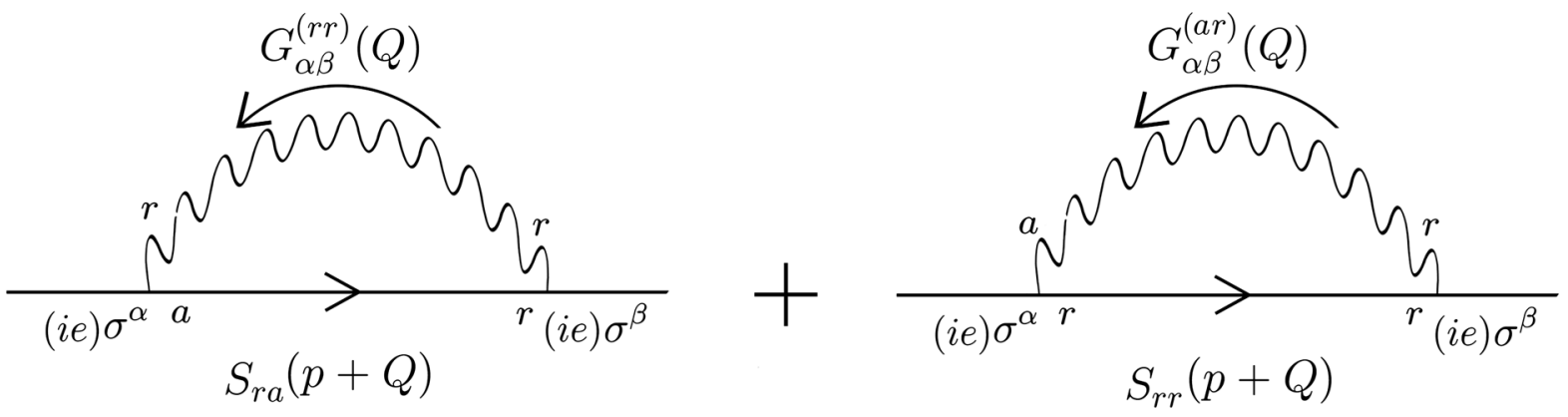

FIG. 8. Real-time Feynman diagrams for the retarded fermion self-energy.

is proportional to the imaginary part of the self-energy, the leading contribution comes from when one of the two loop propagators carries soft momentum (that is, either $Q$ or $p+Q$ ); the soft propagator must then be the HTL resummed propagator, while the other hard propagator is the bare one.

Rotational invariance dictates the self-energy to take a form

$\Sigma^{r a}(p)=A\left(p^{0},|\boldsymbol{p}|\right)+B\left(p^{0},|\boldsymbol{p}|\right) \hat{\boldsymbol{p}} \cdot \vec{\sigma} \equiv i \sum_{s= \pm} \Sigma_{s}^{R}(p) \mathcal{P}_{s}(\boldsymbol{p})$,

where

$$
\Sigma_{s}^{R}(p)=-i\left(A\left(p^{0},|\boldsymbol{p}|\right)+s B\left(p^{0},|\boldsymbol{p}|\right)\right) .
$$

From this and (B2), we have

$$
S^{r a}(p)=\sum_{s} \frac{i}{p^{0}-s|\boldsymbol{p}|+\Sigma_{s}^{R}(p)} \mathcal{P}_{s}(\boldsymbol{p})
$$

In deriving the above, we use the following properties of the projection operators to find the inverse of $S^{r a}(p)$ :

$$
\mathcal{P}_{+}+\mathcal{P}_{-}=1, \quad \mathcal{P}_{ \pm}^{2}=\mathcal{P}_{ \pm}, \quad \mathcal{P}_{+} \mathcal{P}_{-}=\mathcal{P}_{-} \mathcal{P}_{+}=0
$$

By comparing the above expression for $S^{r a}(p)$ with the one in (2.7), we see that the damping rate is given by the imaginary part of retarded self-energy $\Sigma_{s}^{R}(p)$ at on-shell momentum $p^{0}=s|\boldsymbol{p}|$,

$$
\zeta_{\boldsymbol{p}, s}=\left.2 \operatorname{Im}\left[\Sigma_{s}^{R}(p)\right]\right|_{p^{0}=s|\boldsymbol{p}|}
$$

In the following, we will hence only concern ourselves with the imaginary part of $\Sigma_{s}^{R}(p)$. From (B5) and $\operatorname{tr}\left[\mathcal{P}_{ \pm}\right]=1$, we have

$$
\begin{aligned}
\Sigma_{s}^{R}(p)= & (-i) \operatorname{tr}\left[\mathcal{P}_{s}(\boldsymbol{p}) \Sigma^{r a}(p)\right] \\
= & i e^{2} \operatorname{tr}\left[\mathcal{P}_{s}(\boldsymbol{p}) \sigma^{\beta} \int \frac{d^{4} Q}{(2 \pi)^{4}}\right. \\
& \left.\times\left[G_{\alpha \beta}^{r r}(Q) S^{r a}(p+Q)+G_{\alpha \beta}^{a r}(Q) S^{r r}(p+Q)\right] \sigma^{\alpha}\right],
\end{aligned}
$$

which will be the starting point of our computation.

For a soft $p$, if one uses the HTL approximation to the retarded self-energy $\Sigma_{s}^{R}(p)$, the result is the HTL fermion propagator. For a hard $p$, the HTL self-energy is subleading in $e^{2}$, so it can be negligible; moreover, its imaginary part at on-shell momentum $p^{0}=s|\boldsymbol{p}|$, which would give a damping rate that could regularize pinch singularities, vanishes due to kinematic constraints. The leading contribution to the imaginary part of $\Sigma_{s}^{R}(p)$ at on-shell momentum arises when either $Q$ or $p+Q$ is soft, so that the corresponding propagator in the loop is the HTL-resummed one. Calling the case of soft $Q$ the soft-photon contribution, $\zeta_{p, s}^{\mathrm{sp}}$, and the other case of soft $p+Q$ the soft-fermion contribution, $\zeta_{p, s}^{\text {sf }}$, the total damping rate is the sum of the two, $\zeta_{\boldsymbol{p}, s}=\zeta_{\boldsymbol{p}, s}^{\mathrm{sp}}+\zeta_{\boldsymbol{p}, s}^{\mathrm{sf}}$.

Let us discuss $\zeta_{p, s}^{\mathrm{sp}}$ first. Because the fermion propagator is the bare one, we have, after setting the on-shell momentum $p^{0}=s|\boldsymbol{p}|$,

$$
\begin{aligned}
S^{r a}(p+Q)= & \sum_{t= \pm} \frac{i}{s|\boldsymbol{p}|+q^{0}-t|\boldsymbol{p}+\boldsymbol{q}|+i \epsilon} \mathcal{P}_{t}(\boldsymbol{p}+\boldsymbol{q}) \\
= & \sum_{t}\left[P \frac{i}{s|\boldsymbol{p}|+q^{0}-t|\boldsymbol{p}+\boldsymbol{q}|}\right. \\
& \left.+\pi \delta\left(s\left|\boldsymbol{p}+q^{0}-t\right| \boldsymbol{p}+\boldsymbol{q} \mid\right)\right] \mathcal{P}_{t}(\boldsymbol{p}+\boldsymbol{q})
\end{aligned}
$$

where $P$ denotes the principal value. Similarly, 


$$
\begin{aligned}
S^{r r}(p+Q)= & \left(\frac{1}{2}-n_{+}\left(s|\boldsymbol{p}|+q^{0}\right)\right) \\
& \times \sum_{t}(2 \pi) \delta\left(s|\boldsymbol{p}|+q^{0}-t|\boldsymbol{p}+\boldsymbol{q}|\right) \mathcal{P}_{t}(\boldsymbol{p}+\boldsymbol{q}) .
\end{aligned}
$$

Looking at the structure of (B10), we have a spinor trace appearing,

$$
\operatorname{tr}\left[\mathcal{P}_{s}(\boldsymbol{p}) \sigma^{\beta} \mathcal{P}_{t}(\boldsymbol{p}+\boldsymbol{q}) \sigma^{\alpha}\right] \equiv H_{s t}^{\beta \alpha}(\boldsymbol{p}, \boldsymbol{q})
$$

which is a Hermitian matrix in terms of $\alpha \beta$ indices (this can be shown easily using the Hermitian nature of $\sigma^{\alpha}$ and $\mathcal{P}_{ \pm}$). Because

$$
\begin{aligned}
G_{\alpha \beta}^{r r}(Q) & =\left(\frac{1}{2}+n_{B}\left(q^{0}\right)\right)\left(G_{\alpha \beta}^{r a}(Q)-G_{\alpha \beta}^{a r}(Q)\right) \\
& \equiv\left(\frac{1}{2}+n_{B}\left(q^{0}\right)\right) \rho_{\alpha \beta}^{\mathrm{ph}}(Q)
\end{aligned}
$$

is also a Hermitian matrix with $\left(G_{\alpha \beta}^{a r}(Q)\right)^{*}=-G_{\beta \alpha}^{r a}(Q)$, as shown in Appendix A, we see that $H_{s t}^{\beta \alpha}(\boldsymbol{p}, \boldsymbol{q}) G_{\alpha \beta}^{r r}(Q)$ is a real number. Therefore, one sees that the imaginary part of $\Sigma_{s}^{R}(p)$ given in (B10) arises only from the second $\delta$ function term in (B11) when it is used in the first term of (B10). Similarly, from

$$
\left(H_{s t}^{\beta \alpha}(\boldsymbol{p}, \boldsymbol{q}) G_{\alpha \beta}^{a r}(Q)\right)^{*}=-H_{s t}^{\beta \alpha}(\boldsymbol{p}, \boldsymbol{q}) G_{\alpha \beta}^{r a}(Q)
$$

and

$$
\begin{aligned}
& H_{s t}^{\beta \alpha}(\boldsymbol{p}, \boldsymbol{q}) \rho_{\alpha \beta}^{\mathrm{ph}}(Q) \\
& \quad=\left(H_{s t}^{\beta \alpha}(\boldsymbol{p}, \boldsymbol{q}) G_{\alpha \beta}^{r a}(Q)-H_{s t}^{\beta \alpha}(\boldsymbol{p}, \boldsymbol{q}) G_{\alpha \beta}^{a r}(Q)\right),
\end{aligned}
$$

we see that the real part of $H_{s t}^{\beta \alpha}(\boldsymbol{p}, \boldsymbol{q}) G_{\alpha \beta}^{a r}(Q)$ is equal to $-(1 / 2) H_{s t}^{\beta \alpha}(\boldsymbol{p}, \boldsymbol{q}) \rho_{\alpha \beta}^{\mathrm{ph}}(Q)$. With (B12) the imaginary part of $\Sigma_{s}^{R}(p)$ from the second term in (B10) only comes from the real part of $H_{s t}^{\beta \alpha}(\boldsymbol{p}, \boldsymbol{q}) G_{\alpha \beta}^{a r}(Q)$; therefore, we can effectively replace $G_{\alpha \beta}^{a r}(Q)$ appearing in (B10) with $-(1 / 2) \rho_{\alpha \beta}^{\mathrm{ph}}(Q)$ for the purpose of damping rate computation. One then observes that the pieces in (B11) and (B12) that contribute to the damping rate are all proportional to the $\delta$ function $\delta\left(s|\boldsymbol{p}|+q^{0}-t|\boldsymbol{p}+\boldsymbol{q}|\right)$, which has a nonzero support only for $t=s$ because $Q$ is assumed to be soft while $p$ is hard. After collecting all these pieces contributing to the imaginary part of $\Sigma_{s}^{R}(p)$, we finally have after some algebra,

$$
\begin{aligned}
\zeta_{\boldsymbol{p}, s}^{\mathrm{sp}}= & 2 \operatorname{Im}\left[\Sigma_{s}^{R}(p)\right] \\
= & e^{2} \int \frac{d^{4} Q}{(2 \pi)^{4}}\left(n_{B}\left(q^{0}\right)+n_{+}\left(s|\boldsymbol{p}|+q^{0}\right)\right) H_{s s}^{\beta \alpha} \\
& \times(\boldsymbol{p}, \boldsymbol{q}) \rho_{\alpha \beta}^{\mathrm{ph}}(Q)(2 \pi) \delta\left(s|\boldsymbol{p}|+q^{0}-s|\boldsymbol{p}+\boldsymbol{q}|\right),
\end{aligned}
$$

where

$$
\begin{aligned}
H^{\beta \alpha}(\boldsymbol{p}, \boldsymbol{q})_{s s} & =\operatorname{tr}\left[\mathcal{P}_{s}(\boldsymbol{p}) \sigma^{\beta} \mathcal{P}_{s}(\boldsymbol{p}+\boldsymbol{q}) \sigma^{\alpha}\right] \\
& =\Sigma_{s}^{\beta}(\boldsymbol{p}, \boldsymbol{q})\left(\Sigma_{s}^{\alpha}(\boldsymbol{p}, \boldsymbol{q})\right)^{*} \frac{1}{2}(1+\hat{\boldsymbol{p}} \cdot \widehat{\boldsymbol{p}+\boldsymbol{q}})
\end{aligned}
$$

using the notations introduced in (5.4). From the fact that $F_{s}(\boldsymbol{p} ; \boldsymbol{q}, 0)$ introduced in $(5.4)$ is equal to $\frac{1}{2}(1+\hat{\boldsymbol{p}} \cdot \widehat{\boldsymbol{p}+\boldsymbol{q}})$, and recalling our definition of kernel function in (5.10),

$$
\begin{aligned}
\mathcal{K}_{s}(\boldsymbol{p}, 0 ; Q) \equiv & \Sigma_{s}^{\beta}(\boldsymbol{p}, \boldsymbol{q})\left(\Sigma_{s}^{\alpha}(\boldsymbol{p}, \boldsymbol{q})\right)^{*} \rho_{\alpha \beta}^{\mathrm{ph}}(Q) F_{s} \\
& \times(\boldsymbol{p} ; \boldsymbol{q}, 0)\left(n_{+}\left(s|\boldsymbol{p}|+q^{0}\right)+n_{B}\left(q^{0}\right)\right),
\end{aligned}
$$

we see that $\zeta_{\boldsymbol{p}, s}^{\mathrm{sp}}$ is indeed equal to

$\zeta_{\boldsymbol{p}, s}^{\mathrm{sp}}=e^{2} \int \frac{d^{4} Q}{(2 \pi)^{4}} \mathcal{K}_{s}(\boldsymbol{p}, 0 ; Q)(2 \pi) \delta\left(s|\boldsymbol{p}|+q^{0}-s|\boldsymbol{p}+\boldsymbol{q}|\right)$

which is precisely what appears in (5.15) and in the integral equation, which is crucial in order to obtain (5.16).

Although we do not need the value of $\zeta_{p, s}^{\mathrm{sp}}$ in this work, it is easy to compute it from the above expression. From (5.19),

$$
\begin{aligned}
& \int \frac{d^{4} Q}{(2 \pi)^{4}}(2 \pi) \delta\left(s|\boldsymbol{p}|+q^{0}-s|\boldsymbol{p}+\boldsymbol{q}|\right) \\
& =\left.\int_{0}^{\infty} \frac{d|\boldsymbol{q} \| \boldsymbol{q}|}{(2 \pi)} \int_{-|\boldsymbol{q}|}^{|\boldsymbol{q}|} \frac{d q^{0}}{(2 \pi)}\left(1+\frac{s q^{0}}{|\boldsymbol{p}|}\right)\right|_{\hat{\boldsymbol{p}} \cdot \boldsymbol{q} \rightarrow s q^{0}+\frac{\left(q^{0}\right)^{2}-|\boldsymbol{q}|^{2}}{2|\boldsymbol{p}|}},
\end{aligned}
$$

and the small $Q$ expansion of $\mathcal{K}_{s}(\boldsymbol{p}, 0 ; Q)$,

$$
\mathcal{K}_{s}(\boldsymbol{p}, 0 ; Q) \approx \frac{1}{\beta q^{0}}\left(\rho_{L}(Q)+\rho_{T}(Q)\left(1-\frac{\left(q^{0}\right)^{2}}{|\boldsymbol{q}|^{2}}\right)\right),
$$

where $\rho_{L / T}(Q)$ are P-even longitudinal and transverse photon spectral densities defined by

$$
\begin{aligned}
& \rho_{00}^{\mathrm{ph}}(Q)=\rho_{L}(Q), \\
& \rho_{i j}^{\mathrm{ph}}(Q)=\rho_{T}(Q)\left(\delta_{i j}-\frac{\boldsymbol{q}_{i} \boldsymbol{q}_{j}}{|\boldsymbol{q}|^{2}}\right)+i \rho_{\mathrm{odd}}(Q) \epsilon^{i j l} \boldsymbol{q}_{l},
\end{aligned}
$$

we have 


$$
\begin{aligned}
\zeta_{\boldsymbol{p}, s}^{\mathrm{sp}} \approx & e^{2} \int_{0}^{\infty} \frac{d|\boldsymbol{q} \| \boldsymbol{q}|}{(2 \pi)} \int_{-|\boldsymbol{q}|}^{|\boldsymbol{q}|} \frac{d q^{0}}{(2 \pi)} \frac{1}{\beta q^{0}} \\
& \times\left(\rho_{L}(Q)+\rho_{T}(Q)\left(1-\frac{\left(q^{0}\right)^{2}}{|\boldsymbol{q}|^{2}}\right)\right) .
\end{aligned}
$$

The rest of the computation involves using the sum rules for $\rho_{L / T}(Q)$ that can be derived in the same way that we derive the sum rules for the P-odd part in Appendix A [68]. The leading $\log$ arises from the momentum region $|\boldsymbol{q}| \ll m_{D}$, from the transverse part only, for which we have

$$
\begin{aligned}
\int_{-|\boldsymbol{q}|}^{|\boldsymbol{q}|} \frac{d q^{0}}{(2 \pi)} \frac{1}{q^{0}} \rho_{T}(Q) & =\frac{1}{|\boldsymbol{q}|^{2}}+\mathcal{O}\left(\frac{1}{m_{D}^{2}}\right), \\
\int_{-|\boldsymbol{q}|}^{|\boldsymbol{q}|} \frac{d q^{0}}{(2 \pi)} q^{0} \rho_{T}(Q) & =\frac{3|\boldsymbol{q}|^{2}}{5 m_{D}^{2}}+\mathcal{O}\left(\frac{|\boldsymbol{q}|^{4}}{m_{D}^{4}}\right) .
\end{aligned}
$$

This gives

$$
\zeta_{\boldsymbol{p}, s}^{\mathrm{sp}} \approx e^{2} \frac{1}{\beta} \int_{e^{2} T}^{m_{D}} \frac{d|\boldsymbol{q} \| \boldsymbol{q}|}{(2 \pi)} \frac{1}{|\boldsymbol{q}|^{2}} \approx \frac{e^{2} \log (1 / e) T}{2 \pi},
$$

where we set an IR cutoff of order $\Lambda_{\mathrm{IR}} \sim e^{2} T$. Strictly speaking, the $e^{2} T$ (or $g^{2} T$ for non-Abelian theory) magnetic confinement scale exists only for non-Abelian theory, while an Abelian QED that becomes free at $Q \ll m_{f}$ does not possess any IR cutoff. In this case, the damping rate $\zeta_{\boldsymbol{p}, s}^{\mathrm{sp}}$ is not a useful concept [73], and the effective IR cutoff is provided by the time scale one is looking at, so the hard fermions decay in time $t$ as [73]

$$
\left.e^{-\zeta_{p, s}^{\mathrm{p}} t / 2}\right|_{\Lambda_{\mathrm{IR}} \sim 1 / t} \sim\left(m_{D} t\right)^{-\frac{e^{2} T}{4 \pi} t} .
$$

Because we are ultimately interested in QCD (see our discussion in Sec. VI), we do not worry about this any longer. In addition, in realistic situations, the free nature of QED at $Q \ll e T$ means that this scale is never thermalized anyway. As the damping rate arises from the scattering of fermions with thermally excited soft photons in this scale [recall $n_{B}\left(q^{0}\right) \sim 1 / q^{0}$ term in the above], we would not have these contributions in realistic situations in any case. This also justifies our use of $\Lambda_{\mathrm{IR}} \sim e^{2} T$ in the above.

Let us next compute the soft-fermion contribution to the damping rate, $\zeta_{p, s}^{\text {sf }}$, with our main objective being to find a linear $s \mu$ dependence. Since $p+Q$ is soft, it is convenient to shift the loop momentum $Q \rightarrow Q-p$ to have

$$
\begin{aligned}
\Sigma_{s}^{R}(p)= & i e^{2} \operatorname{tr}\left[\mathcal { P } _ { s } ( \boldsymbol { p } ) \sigma ^ { \beta } \int \frac { d ^ { 4 } Q } { ( 2 \pi ) ^ { 4 } } \left[G_{\alpha \beta}^{r r}(Q-p) S^{r a}(Q)\right.\right. \\
& \left.\left.+G_{\alpha \beta}^{a r}(Q-p) S^{r r}(Q)\right] \sigma^{\alpha}\right]
\end{aligned}
$$

where $Q$ is now soft, and we need to use HTL-resummed fermion propagators while the bare propagators are used for photon propagators. The HTL-resummed fermion $r a$ propagator is written as

$$
S^{r a}(Q)=\sum_{t} \frac{i}{q^{0}-t|\boldsymbol{q}|+\Sigma_{t}^{R, \mathrm{HTL}}(Q)} \mathcal{P}_{t}(\boldsymbol{q}),
$$

where $\Sigma_{t}^{R, \mathrm{HTL}}(Q)$ is the HTL self-energy. An explicit computation gives [in fact, one uses the same expression (B28) with both $Q$ and $p+Q$ now hard]

$$
\begin{aligned}
& \Sigma_{t}^{R, \mathrm{HTL}}(Q) \\
& \quad=-\frac{m_{f}^{2}}{4|\boldsymbol{q}|}\left(2 t+\left(1-t \frac{q^{0}}{|\boldsymbol{q}|}\right) \log \left(\frac{q^{0}+|\boldsymbol{q}|+i \epsilon}{q^{0}-|\boldsymbol{q}|+i \epsilon}\right)\right),
\end{aligned}
$$

where

$$
m_{f}^{2}=\frac{e^{2}}{4}\left(T^{2}+\frac{\mu^{2}}{\pi^{2}}\right)
$$

is the asymptotic thermal mass of fermions for a single Weyl fermion system. Since $\mu$ dependence is only quadratic for $\Sigma_{t}^{R, \mathrm{HTL}}(Q)$, we can ignore this dependence in the HTL self-energy to use the $\mu=0$ result of $\Sigma_{t}^{R, \mathrm{HTL}}(Q)$. This means that $S^{r a}(Q)$ and $S^{a r}=-\left(S^{r a}(Q)\right)^{\dagger}$ can be replaced by their $\mu=0$ values up to linear order in the chemical potential $\mu$, which is the order of our interest. On the other hand, the rr propagator, which is given by

$$
S^{r r}(Q)=\left(\frac{1}{2}-n_{+}\left(q^{0}\right)\right)\left(S^{r a}(Q)-S^{a r}(Q)\right),
$$

does contain a linear $\mu$ dependence via its statistical factor in front, $n_{+}\left(q^{0}\right)$. We will indeed shortly find that this will be the (only) source of the final $s \mu$ dependence of $\zeta_{\boldsymbol{p}, s}^{\text {sf }}$.

Becase the HTL-resummed $r a$ propagator (B29) is analytic in the upper $q^{0}$ complex plane, one can introduce real spectral densities $\rho_{ \pm}(Q)$ by

$$
\begin{aligned}
\frac{1}{q^{0}-t|\boldsymbol{q}|+\sum_{t}^{R, \mathrm{HTL}}(Q)}= & \int_{-\infty}^{\infty} \frac{d \omega}{(2 \pi)} \frac{\rho_{t}(\omega, \boldsymbol{q})}{q^{0}-\omega+i \epsilon} \\
= & P \int_{-\infty}^{\infty} \frac{d \omega}{(2 \pi)} \frac{\rho_{t}(\omega, \boldsymbol{q})}{q^{0}-\omega} \\
& -\frac{i}{2} \rho_{t}\left(q^{0}, \boldsymbol{q}\right),
\end{aligned}
$$

or, equivalently,

$$
\rho_{t}(Q)=-2 \operatorname{Im}\left[\frac{1}{q^{0}-t|\boldsymbol{q}|+\Sigma_{t}^{R, \mathrm{HTL}}(Q)}\right],
$$

in terms of which we have 


$$
S^{r r}(Q)=\left(\frac{1}{2}-n_{+}\left(q^{0}\right)\right) \sum_{t} \rho_{t}(Q) \mathcal{P}_{t}(\boldsymbol{q})
$$

Introducing

$$
L_{s t}^{\beta \alpha}(\boldsymbol{p}, \boldsymbol{q}) \equiv \operatorname{tr}\left[\mathcal{P}_{s}(\boldsymbol{p}) \sigma^{\beta} \mathcal{P}_{t}(\boldsymbol{q}) \sigma^{\alpha}\right]
$$

which is a Hermitian matrix, a similar discussion to that which we have above leads us to replace

$$
S^{r a}(Q) \rightarrow \frac{1}{2} \rho_{t}(Q) \mathcal{P}_{t}(\boldsymbol{q})
$$

for computing the imaginary part of $\Sigma_{s}^{R}(p)$ in (B28).

On the other hand, the hard photon propagators in (B28) are bare ones. In the Coulomb gauge we have

$$
G_{00}^{r a}(p)=\frac{i}{|\boldsymbol{p}|^{2}}, \quad G_{i j}^{r a}(p)=\frac{-i P_{i j}^{T}(\boldsymbol{p})}{-\left(p^{0}+i \epsilon\right)^{2}+|\boldsymbol{p}|^{2}},
$$

where $P_{i j}^{T}(\boldsymbol{p})=\delta_{i j}-\hat{\boldsymbol{p}}_{i} \hat{\boldsymbol{p}}_{j}$ is the transverse projection operator, from which we have the bare photon spectral density as

$$
\begin{aligned}
& \rho_{00}^{\mathrm{ph}}(p)=0, \\
& \rho_{i j}^{\mathrm{ph}}(p)=(2 \pi) P_{i j}^{T}(\boldsymbol{p}) \operatorname{sign}\left(p^{0}\right) \delta\left(\left(p^{0}\right)^{2}-|\boldsymbol{p}|^{2}\right),
\end{aligned}
$$

with $G_{\alpha \beta}^{r r}(p)=\left(1 / 2+n_{B}\left(p^{0}\right)\right) \rho_{\alpha \beta}^{\mathrm{ph}}(p)$. As before, for the imaginary part of $\Sigma_{s}^{R}(p)$ in (B28), we can replace

$$
G_{\alpha \beta}^{a r}(Q-p) \rightarrow-\frac{1}{2} \rho_{\alpha \beta}^{\mathrm{ph}}(Q-p)
$$

Collecting all these elements, the expression for $\zeta_{p, s}^{\text {sf }}$ becomes

$$
\begin{aligned}
\zeta_{\boldsymbol{p}, s}^{\mathrm{sf}}= & e^{2} \sum_{t= \pm} \int \frac{d^{4} Q}{(2 \pi)^{4}} L_{s t}^{j i}(\boldsymbol{p}, \boldsymbol{q}) P_{i j}^{T}(\boldsymbol{q}-\boldsymbol{p})\left(n_{B}\left(q^{0}-s|\boldsymbol{p}|\right)\right. \\
& \left.+n_{+}\left(q^{0}\right)\right) \rho_{t}(Q) \operatorname{sign}\left(q^{0}-s|\boldsymbol{p}|\right)(2 \pi) \delta((s|\boldsymbol{p}| \\
& \left.\left.-q^{0}\right)^{2}-|\boldsymbol{p}-\boldsymbol{q}|^{2}\right) .
\end{aligned}
$$

Because $Q$ is soft while $p$ is hard, we have $\operatorname{sign}\left(q^{0}-s|\boldsymbol{p}|\right)=$ $-s$ and

$$
\begin{aligned}
& \delta\left(\left(s|\boldsymbol{p}|-q^{0}\right)^{2}-|\boldsymbol{p}-\boldsymbol{q}|^{2}\right) \\
& \quad=\frac{1}{2\left(|\boldsymbol{p}|-s q^{0}\right)} \delta\left(s|\boldsymbol{p}|-q^{0}-s|\boldsymbol{p}-\boldsymbol{q}|\right),
\end{aligned}
$$

and, using the identity

$$
\begin{aligned}
& n_{B}\left(q^{0}-s|\boldsymbol{p}|\right)+n_{+}\left(q^{0}\right) \\
& \quad=(-s)\left(n_{B}\left(|\boldsymbol{p}|-s q^{0}\right)+n_{-s}\left(-s q^{0}\right)\right),
\end{aligned}
$$

we have

$$
\begin{aligned}
\zeta_{p, s}^{\mathrm{sf}}= & e^{2} \sum_{t= \pm} \int \frac{d^{4} Q}{(2 \pi)^{4}} L_{s t}^{j i}(\boldsymbol{p}, \boldsymbol{q}) P_{i j}^{T}(\boldsymbol{q}-\boldsymbol{p})\left(n_{B}\left(|\boldsymbol{p}|-s q^{0}\right)\right. \\
& \left.+n_{-s}\left(-s q^{0}\right)\right) \rho_{t}(Q) \frac{1}{2\left(|\boldsymbol{p}|-s q^{0}\right)} \\
& \times(2 \pi) \delta\left(s|\boldsymbol{p}|-q^{0}-s|\boldsymbol{p}-\boldsymbol{q}|\right) .
\end{aligned}
$$

It is straightforward to compute the leading log part of the above integral by expanding the integrand in powers of $Q / T$ or $Q /|\boldsymbol{p}|$, the same procedure we have used several times before. From $L_{s t}^{j i}(\boldsymbol{p}, \boldsymbol{q}) P_{i j}^{T}(\boldsymbol{q}-\boldsymbol{p}) \approx 1-s t \frac{\hat{p} \cdot \boldsymbol{q}}{|\boldsymbol{q}|}+\mathcal{O}(Q)=$ $1-t \frac{q^{0}}{|\boldsymbol{q}|}+\mathcal{O}(Q) \quad$ and $\quad n_{B}\left(|\boldsymbol{p}|-s q^{0}\right)+n_{-s}\left(-s q^{0}\right) \approx$ $n_{B}(|\boldsymbol{p}|)+n_{-s}(0)+\mathcal{O}(Q)$, the leading log comes from the expression

$$
\begin{aligned}
\zeta_{\boldsymbol{p}, s}^{\mathrm{sf}}= & \frac{e^{2}}{2|\boldsymbol{p}|}\left(n_{B}(|\boldsymbol{p}|)+n_{-s}(0)\right) \sum_{t} \int_{0}^{\infty} \frac{d|\boldsymbol{q} \| \boldsymbol{q}|}{2 \pi} \\
& \times \int_{-|\boldsymbol{q}|}^{|\boldsymbol{q}|} \frac{d q^{0}}{2 \pi}\left(1-t \frac{q^{0}}{|\boldsymbol{q}|}\right) \rho_{t}(Q),
\end{aligned}
$$

and, using the sum rules ${ }^{10}$

$$
\begin{aligned}
& J_{0}^{ \pm}=\frac{m_{f}^{2}}{4|\boldsymbol{q}|^{2}}\left(\log \frac{4|\boldsymbol{q}|^{2}}{m_{f}^{2}}-1\right), \\
& J_{1}^{ \pm}= \pm \frac{m_{f}^{2}}{4|\boldsymbol{q}|}\left(\log \frac{4|\boldsymbol{q}|^{2}}{m_{f}^{2}}-3\right),
\end{aligned}
$$

where

$$
J_{n}^{ \pm} \equiv \int_{-|q|}^{|q|} \frac{d q^{0}}{2 \pi}\left(q^{0}\right)^{n} \rho_{ \pm}(Q),
$$

we finally have

$$
\zeta_{\boldsymbol{p}, s}^{\mathrm{sf}}=\frac{e^{2}}{4 \pi} \frac{m_{f}^{2} \log (1 / e)}{|\boldsymbol{p}|}\left(n_{B}(|\boldsymbol{p}|)+n_{-s}(0)\right) .
$$

There exists $s \mu$ dependence in the result (B48) via $n_{-s}(0)=1-n_{s}(0) \approx 1 / 2-(1 / 4) s \beta \mu$, which can be easily understood as follows. The soft-fermion contribution to the damping rate comes from the process where a hard fermion (of type $s$ ) becomes a soft fermion (of the same type $s$ ) by

\footnotetext{
${ }^{10}$ We point out that what is called $m_{f}^{2}$ in Ref. [59] is in fact plasmino frequency $\omega_{f}^{2}$, which is equal to $m_{f}^{2} / 2$ in terms of asymptotic thermal mass $m_{f}^{2}$.
} 
emitting a hard photon with almost the same momentum. The rate is proportional to $\left(n_{B}(|\boldsymbol{p}|)+1\right)\left(1-n_{s}(0)\right)$ where $\left(1-n_{s}(0)\right)$ is the Pauli blocking factor of the final softfermion state, where we can set zero for soft momentum at leading order in coupling. In a similar process, a hard fermion (of type $s$ ) meets with a soft antifermion (of the type $-s)$ to become a hard photon; this rate is proportional to $\left(n_{B}(|\boldsymbol{p}|)+1\right) n_{-s}(0)$ where $n_{-s}(0)$ is the number density of the soft antifermion. The time-reversed processes also add up to the damping rate, which is a property of the fermionic case. These are each proportional to $n_{B}(|\boldsymbol{p}|) n_{s}(0)$ and $n_{B}(|\boldsymbol{p}|)\left(1-n_{-s}(0)\right)$. Using $n_{-s}(0)+n_{s}(0)=1$, the total sum can be found to be

$$
\begin{aligned}
& \left(n_{B}(|\boldsymbol{p}|)+1\right)\left(1-n_{s}(0)\right)+\left(n_{B}(|\boldsymbol{p}|)+1\right) n_{-s}(0) \\
& \quad+n_{B}(|\boldsymbol{p}|) n_{s}(0)+n_{B}(|\boldsymbol{p}|)\left(1-n_{-s}(0)\right) \\
& \quad=2\left(n_{B}(|\boldsymbol{p}|)+n_{-s}(0)\right),
\end{aligned}
$$

which nicely explains our result (B48).

\section{APPENDIX C: EXPRESSION OF $\boldsymbol{F}_{s}(p, q ; k)$}

The function $F_{s}(\boldsymbol{p}, \boldsymbol{q} ; \boldsymbol{k})$ is given by

$F_{s}(\boldsymbol{p}, \boldsymbol{q} ; \boldsymbol{k})=\frac{A}{4(1+\hat{\boldsymbol{p}} \cdot \widehat{\boldsymbol{p}+\boldsymbol{k}})|\boldsymbol{p}\|\boldsymbol{p}+\boldsymbol{q}\| \boldsymbol{p}+\boldsymbol{k} \| \boldsymbol{p}+\boldsymbol{q}+\boldsymbol{k}|}$,

(C1)

where

$$
\begin{aligned}
A= & \left(|\boldsymbol{p} \| \boldsymbol{p}+\boldsymbol{q}|+|\boldsymbol{p}|^{2}+\boldsymbol{p} \cdot \boldsymbol{q}\right)(|\boldsymbol{p}+\boldsymbol{k} \| \boldsymbol{p}+\boldsymbol{k}+\boldsymbol{q}| \\
& \left.+|\boldsymbol{p}+\boldsymbol{k}|^{2}+(\boldsymbol{p}+\boldsymbol{k}) \cdot \boldsymbol{q}\right)+(|\boldsymbol{p} \| \boldsymbol{p}+\boldsymbol{k}| \\
& \left.+|\boldsymbol{p}|^{2}+\boldsymbol{p} \cdot \boldsymbol{k}\right)\left(|\boldsymbol{p}+\boldsymbol{q} \| \boldsymbol{p}+\boldsymbol{q}+\boldsymbol{k}|+|\boldsymbol{p}+\boldsymbol{q}|^{2}\right. \\
& +(\boldsymbol{p}+\boldsymbol{q}) \cdot \boldsymbol{k})-(|\boldsymbol{p} \| \boldsymbol{p}+\boldsymbol{q}+\boldsymbol{k}|-\boldsymbol{p} \cdot(\boldsymbol{p}+\boldsymbol{q}+\boldsymbol{k})) \\
& \times(|\boldsymbol{p}+\boldsymbol{q} \| \boldsymbol{p}+\boldsymbol{k}|-(\boldsymbol{p}+\boldsymbol{q}) \cdot(\boldsymbol{p}+\boldsymbol{k})) \\
& +i s(|\boldsymbol{p}|+|\boldsymbol{p}+\boldsymbol{q}|+|\boldsymbol{p}+\boldsymbol{k}| \\
& +|\boldsymbol{p}+\boldsymbol{q}+\boldsymbol{k}|) \epsilon^{i j l} \boldsymbol{p}^{i} \boldsymbol{q}^{j} \boldsymbol{k}^{l} .
\end{aligned}
$$

For the reasons mentioned in the main text, we are only interested in this quantity to linear order in the external momentum $\boldsymbol{k}$ and to second order in the loop momentum $\boldsymbol{q}$. To this order the function $F_{s}(\boldsymbol{p}, \boldsymbol{q} ; \boldsymbol{k})$ is given by

$$
\begin{aligned}
& F_{s}(\boldsymbol{p}, \boldsymbol{q} ; \boldsymbol{k}) \sim 1+\frac{1}{4|\boldsymbol{p}|^{2}}\left((\boldsymbol{p} \cdot \boldsymbol{q})^{2}-|\boldsymbol{q}|^{2}\right)+\frac{1}{2|\boldsymbol{p}|^{3}} \operatorname{sic}^{i j l} \boldsymbol{p}_{i} \boldsymbol{q}_{j} \boldsymbol{k}_{l} \\
& \quad+\frac{1}{4|\boldsymbol{p}|^{4}}\left(\boldsymbol{p} \cdot \boldsymbol{k}\left(|\boldsymbol{q}|^{2}-2(\boldsymbol{p} \cdot \boldsymbol{q})^{2}\right)+(\boldsymbol{p} \cdot \boldsymbol{k})(\boldsymbol{p} \cdot \boldsymbol{q})\right. \\
& \left.\quad-3 \operatorname{si\epsilon }{ }^{i j l} \boldsymbol{p}_{i} \boldsymbol{q}_{j} \boldsymbol{k}_{l}(\boldsymbol{p} \cdot \boldsymbol{q})\right) .
\end{aligned}
$$

\section{APPENDIX D: $\mu^{2}$ CORRECTION TO ELECTRIC CONDUCTIVITY}

Our analysis in this work contains all the necessary ingredients to compute the full $\mu^{2}$ correction to the usual Peven electric conductivity at leading log order. The electric conductivity is given from $\chi_{s}(|\boldsymbol{p}|)$ by

$$
\sigma=-\left.\frac{e^{2}}{3} \int \frac{d^{3} \boldsymbol{p}}{(2 \pi)^{3}} \sum_{s= \pm}\left(\frac{d n_{+}\left(p^{0}\right)}{d p^{0}}\right)\right|_{p^{0}=s|\boldsymbol{p}|} \chi_{s}(|\boldsymbol{p}|),
$$

where $\chi_{s}(|\boldsymbol{p}|)$ satisfies the second order differential equation written in (5.32),

$$
\begin{aligned}
\zeta_{\boldsymbol{p}, s}^{\mathrm{sf}} \chi_{s}(|\boldsymbol{p}|)= & s-\frac{e^{2} m_{D}^{2} \log (1 / e)}{4 \pi}\left(\frac{1}{\beta|\boldsymbol{p}|^{2}} \chi_{s}(|\boldsymbol{p}|)\right. \\
& \left.-\left(\frac{1}{\beta|\boldsymbol{p}|}+n_{s}(|\boldsymbol{p}|)-\frac{1}{2}\right) \chi_{s}^{\prime}(|\boldsymbol{p}|)-\frac{1}{2 \beta} \chi_{s}^{\prime \prime}(|\boldsymbol{p}|)\right),
\end{aligned}
$$

where the soft-fermion contribution to the damping rate $\zeta_{p, s}^{\text {sf }}$ is given by (B48),

$$
\zeta_{\boldsymbol{p}, s}^{\mathrm{sf}}=\frac{e^{2}}{4 \pi} \frac{m_{f}^{2} \log (1 / e)}{|\boldsymbol{p}|}\left(n_{B}(|\boldsymbol{p}|)+n_{-s}(0)\right) .
$$

To correctly take into account $\mu^{2}$ corrections, we need to restore full expressions for $m_{D}^{2}$ and $m_{f}^{2}$, including $\mu^{2}$ corrections,

$$
m_{D}^{2}=\frac{e^{2}}{6}\left(T^{2}+\frac{3 \mu^{2}}{\pi^{2}}\right), \quad m_{f}^{2}=\frac{e^{2}}{4}\left(T^{2}+\frac{\mu^{2}}{\pi^{2}}\right) .
$$

Also, we have to expand $n_{s}(|\boldsymbol{p}|)$ in (D2) and $n_{-s}(0)$ in (D3) up to second order in $\mu$,

$$
\begin{aligned}
n_{s}(|\boldsymbol{p}|)= & n_{0}(|\boldsymbol{p}|-s \mu) \approx n_{0}(|\boldsymbol{p}|)-s \mu n_{0}^{\prime}(|\boldsymbol{p}|) \\
& +\frac{1}{2} n_{0}^{\prime \prime}(|\boldsymbol{p}|) \mu^{2},
\end{aligned}
$$

and $n_{-s}(0) \approx 1 / 2-s \beta \mu / 4+\mathcal{O}\left(\mu^{3}\right)$, where $n_{0}(x) \equiv 1 /$ $\left(e^{\beta x}+1\right)$. The resulting $\chi_{s}(|\boldsymbol{p}|)$ should be found up to $\mu^{2}$ order as

$$
\chi_{s}(|\boldsymbol{p}|)=s \chi_{(0)}(|\boldsymbol{p}|)+\mu \chi_{(1)}(|\boldsymbol{p}|)+s \mu^{2} \chi_{(2)}(|\boldsymbol{p}|)+\mathcal{O}\left(\mu^{3}\right),
$$

where $\left(\chi_{(0)}, \chi_{(1)}, \chi_{(2)}\right)$ can be obtained from (D2) by solving it order by order in $\mu$. 
[1] D. Kharzeev and A. Zhitnitsky, Charge separation induced by P-odd bubbles in QCD matter, Nucl. Phys. A797, 67 (2007).

[2] D. E. Kharzeev, L. D. McLerran, and H. J. Warringa, The effects of topological charge change in heavy ion collisions: 'Event by event $\mathrm{P}$ and $C P$ violation', Nucl. Phys. A803, 227 (2008).

[3] K. Fukushima, D. E. Kharzeev, and H. J. Warringa, The chiral magnetic effect, Phys. Rev. D 78, 074033 (2008).

[4] D. T. Son and A. R. Zhitnitsky, Quantum anomalies in dense matter, Phys. Rev. D 70, 074018 (2004).

[5] M. A. Metlitski and A. R. Zhitnitsky, Anomalous axion interactions and topological currents in dense matter, Phys. Rev. D 72, 045011 (2005).

[6] D. T. Son and P. Surowka, Hydrodynamics with Triangle Anomalies, Phys. Rev. Lett. 103, 191601 (2009).

[7] J. Erdmenger, M. Haack, M. Kaminski, and A. Yarom, Fluid dynamics of R-charged black holes, J. High Energy Phys. 01 (2009) 055.

[8] N. Banerjee, J. Bhattacharya, S. Bhattacharyya, S. Dutta, R. Loganayagam, and P. Surowka, Hydrodynamics from charged black branes, J. High Energy Phys. 01 (2011) 094.

[9] D. E. Kharzeev and H. J. Warringa, Chiral magnetic conductivity, Phys. Rev. D 80, 034028 (2009).

[10] K. Landsteiner, E. Megias, and F. Pena-Benitez, Gravitational Anomaly and Transport, Phys. Rev. Lett. 107, 021601 (2011).

[11] S. Golkar and D. T. Son, (Non)-renormalization of the chiral vortical effect coefficient, J. High Energy Phys. 02 (2015) 169.

[12] D. Satow and H. U. Yee, Chiral magnetic effect at weak coupling with relaxation dynamics, Phys. Rev. D 90, 014027 (2014).

[13] H.-U. Yee, Holographic chiral magnetic conductivity, J. High Energy Phys. 11 (2009) 085.

[14] A. Rebhan, A. Schmitt, and S. A. Stricker, Anomalies and the chiral magnetic effect in the Sakai-Sugimoto model, J. High Energy Phys. 01 (2010) 026.

[15] A. Gynther, K. Landsteiner, F. Pena-Benitez, and A. Rebhan, Holographic anomalous conductivities and the chiral magnetic effect, J. High Energy Phys. 02 (2011) 110.

[16] C. Hoyos, T. Nishioka, and A. O'Bannon, A chiral magnetic effect from AdS/CFT with flavor, J. High Energy Phys. 10 (2011) 084.

[17] I. Amado, K. Landsteiner, and F. Pena-Benitez, Anomalous transport coefficients from Kubo formulas in Holography, J. High Energy Phys. 05 (2011) 081.

[18] N. Banerjee, J. Bhattacharya, S. Bhattacharyya, S. Jain, S. Minwalla, and T. Sharma, Constraints on fluid dynamics from equilibrium partition functions, J. High Energy Phys. 09 (2012) 046.

[19] K. Jensen, Triangle anomalies, thermodynamics, and hydrodynamics, Phys. Rev. D 85, 125017 (2012).

[20] K. Jensen, R. Loganayagam, and A. Yarom, Thermodynamics, gravitational anomalies and cones, J. High Energy Phys. 02 (2013) 088.

[21] P. V. Buividovich, M. N. Chernodub, E. V. Luschevskaya, and M. I. Polikarpov, Numerical evidence of chiral magnetic effect in lattice gauge theory, Phys. Rev. D 80, 054503 (2009).
[22] M. Abramczyk, T. Blum, G. Petropoulos, and R. Zhou, Chiral magnetic effect in $2+1$ flavor QCD + QED, Proc. Sci., LAT2009 (2009) 181.

[23] A. Yamamoto, Chiral Magnetic Effect in Lattice QCD with a Chiral Chemical Potential, Phys. Rev. Lett. 107, 031601 (2011).

[24] P. V. Buividovich, Anomalous transport with overlap fermions, Nucl. Phys. A925, 218 (2014).

[25] G. S. Bali, F. Bruckmann, G. Endrdi, Z. Fodor, S. D. Katz, and A. Schfer, Local $C P$-violation and electric charge separation by magnetic fields from lattice QCD, J. High Energy Phys. 04 (2014) 129.

[26] B. I. Abelev et al. (STAR Collaboration), Azimuthal Charged-Particle Correlations and Possible Local Strong Parity Violation, Phys. Rev. Lett. 103, 251601 (2009).

[27] G. Wang (STAR Collaboration), Search for chiral magnetic effects in high-energy nuclear collisions, Nucl. Phys. A904905, 248c (2013).

[28] H. Ke (STAR Collaboration), Charge asymmetry dependency of $\pi^{+} / \pi^{-}$elliptic flow in $\mathrm{Au}+\mathrm{Au}$ collisions at $\sqrt{s_{N N}}=200 \mathrm{GeV}$, J. Phys. Conf. Ser. 389, 012035 (2012).

[29] Q.-Y. Shou et al. (STAR Collaboration), Charge asymmetry dependency of $/ \mathrm{K}$ anisotropic flow in $\mathrm{U}+\mathrm{U}=193 \mathrm{GeV}$ and $\mathrm{Au}+\mathrm{Au}=200 \mathrm{GeV}$ collisions at STAR, J. Phys. Conf. Ser. 509, 012033 (2014).

[30] L. Adamczyk et al. (STAR Collaboration), Observation of Charge Asymmetry Dependence of Pion Elliptic Flow and the Possible Chiral Magnetic Wave in Heavy-Ion Collisions, Phys. Rev. Lett. 114, 252302 (2015).

[31] I. Selyuzhenkov (ALICE Collaboration), Anisotropic flow and other collective phenomena measured in $\mathrm{Pb}-\mathrm{Pb}$ collisions with ALICE at the LHC, Prog. Theor. Phys. Suppl. 193, 153 (2012).

[32] D. E. Kharzeev and D. T. Son, Testing the Chiral Magnetic and Chiral Vortical Effects in Heavy Ion Collisions, Phys. Rev. Lett. 106, 062301 (2011).

[33] Y. Jiang, X. G. Huang, and J. Liao, Chiral vortical wave and induced flavor charge transport in a rotating quark-gluon plasma, arXiv:1504.03201.

[34] D. E. Kharzeev and H.-U. Yee, Chiral magnetic wave, Phys. Rev. D 83, 085007 (2011).

[35] G. M. Newman, Anomalous hydrodynamics, J. High Energy Phys. 01 (2006) 158.

[36] Y. Burnier, D. E. Kharzeev, J. Liao, and H.-U. Yee, Chiral Magnetic Wave at Finite Baryon Density and the Electric Quadrupole Moment of Quark-Gluon Plasma in Heavy Ion Collisions, Phys. Rev. Lett. 107, 052303 (2011).

[37] E. V. Gorbar, V. A. Miransky, and I. A. Shovkovy, Normal ground state of dense relativistic matter in a magnetic field, Phys. Rev. D 83, 085003 (2011).

[38] H.-U. Yee and Y. Yin, Realistic implementation of chiral magnetic wave in heavy ion collisions, Phys. Rev. C 89, 044909 (2014).

[39] Q. Li, D. E. Kharzeev, C. Zhang, Y. Huang, I. Pletikosic, A. V. Fedorov, R. D. Zhong, J. A. Schneeloch et al., Observation of the chiral magnetic effect in ZrTe5, arXiv:1412.6543.

[40] D. E. Kharzeev and H.-U. Yee, Anomalies and time reversal invariance in relativistic hydrodynamics: the second order and higher dimensional formulations, Phys. Rev. D 84, 045025 (2011). 
[41] R. Loganayagam, Anomaly induced transport in arbitrary dimensions, arXiv:1106.0277.

[42] R. Loganayagam and P. Surowka, Anomaly/Transport in an ideal Weyl gas, J. High Energy Phys. 04 (2012) 097.

[43] N. Banerjee, S. Dutta, S. Jain, R. Loganayagam, and T. Sharma, Constraints on anomalous fluid in arbitrary dimensions, J. High Energy Phys. 03 (2013) 048.

[44] K. Jensen, R. Loganayagam, and A. Yarom, Anomaly inflow and thermal equilibrium, J. High Energy Phys. 05 (2014) 134.

[45] K. Jensen, R. Loganayagam, and A. Yarom, Chern-Simons terms from thermal circles and anomalies, J. High Energy Phys. 05 (2014) 110.

[46] H. U. Yee, Chiral magnetic and vortical effects in higher dimensions at weak coupling, Phys. Rev. D 90, 065021 (2014).

[47] S. Bhattacharyya, J. R. David, and S. Thakur, Second order transport from anomalies, J. High Energy Phys. 01 (2014) 010.

[48] E. Megias and F. Pena-Benitez, Holographic gravitational anomaly in first and second order hydrodynamics, J. High Energy Phys. 05 (2013) 115.

[49] D. T. Son and N. Yamamoto, Berry Curvature, Triangle Anomalies, and the Chiral Magnetic Effect in Fermi Liquids, Phys. Rev. Lett. 109, 181602 (2012).

[50] M. A. Stephanov and Y. Yin, Chiral Kinetic Theory, Phys. Rev. Lett. 109, 162001 (2012).

[51] J.-H. Gao, Z.-T. Liang, S. Pu, Q. Wang, and X.-N. Wang, Chiral Anomaly and Local Polarization Effect from Quantum Kinetic Approach, Phys. Rev. Lett. 109, 232301 (2012).

[52] J. Y. Chen, D. T. Son, M. A. Stephanov, H. U. Yee, and Y. Yin, Lorentz Invariance in Chiral Kinetic Theory, Phys. Rev. Lett. 113, 182302 (2014).

[53] J. Y. Chen, D. T. Son, and M. A. Stephanov, Collisions in Chiral Kinetic Theory, Phys. Rev. Lett. 115, 021601 (2015).

[54] G. Baym, H. Monien, C. J. Pethick, and D. G. Ravenhall, Transverse Interactions and Transport in Relativistic QuarkGluon and Electromagnetic Plasmas, Phys. Rev. Lett. 64, 1867 (1990).

[55] P. B. Arnold, G. D. Moore, and L. G. Yaffe, Transport coefficients in high temperature gauge theories. 1. Leading log results, J. High Energy Phys. 11 (2000) 001.

[56] X. G. Huang and J. Liao, Axial Current Generation from Electric Field: Chiral Electric Separation Effect, Phys. Rev. Lett. 110, 232302 (2013).

[57] S. Jeon, Hydrodynamic transport coefficients in relativistic scalar field theory, Phys. Rev. D 52, 3591 (1995).
[58] M. A. Valle Basagoiti, Transport coefficients and ladder summation in hot gauge theories, Phys. Rev. D 66, 045005 (2002).

[59] G. Aarts and J. M. Martinez Resco, Ward identity and electrical conductivity in hot QED, J. High Energy Phys. 11, (2002) 022.

[60] J.-S. Gagnon and S. Jeon, Leading order calculation of electric conductivity in hot quantum electrodynamics from diagrammatic methods, Phys. Rev. D 75, 025014 (2007); 76, 089902(E) (2007).

[61] R. D. Pisarski, Scattering Amplitudes in Hot Gauge Theories, Phys. Rev. Lett. 63, 1129 (1989).

[62] E. Braaten and R. D. Pisarski, Resummation and Gauge Invariance of the Gluon Damping Rate in Hot QCD, Phys. Rev. Lett. 64, 1338 (1990).

[63] A. Selikhov and M. Gyulassy, Color diffusion and conductivity in a quark-gluon plasma, Phys. Lett. B 316, 373 (1993).

[64] D. Bodeker, On the effective dynamics of soft non-Abelian gauge fields at finite temperature, Phys. Lett. B 426, 351 (1998).

[65] P. B. Arnold, D. T. Son, and L. G. Yaffe, Effective dynamics of hot, soft non-Abelian gauge fields. Color conductivity and $\log$ (1/alpha) effects, Phys. Rev. D 59, 105020 (1999).

[66] Y. Matsuo, S. J. Sin, S. Takeuchi, and T. Tsukioka, Magnetic conductivity and Chern-Simons term in holographic hydrodynamics of charged AdS black hole, J. High Energy Phys. 04 (2010) 071.

[67] B. Sahoo and H. U. Yee, Holographic chiral shear waves from anomaly, Phys. Lett. B 689, 206 (2010).

[68] J.P. Blaizot and E. Iancu, The quark gluon plasma: Collective dynamics and hard thermal loops, Phys. Rep. 359, 355 (2002).

[69] J. M. Martinez Resco and M. A. Valle Basagoiti, Color conductivity and ladder summation in hot QCD, Phys. Rev. D 63, 056008 (2001).

[70] Y. Akamatsu and N. Yamamoto, Chiral Langevin theory for non-Abelian plasmas, Phys. Rev. D 90, 125031 (2014).

[71] D. T. Son and N. Yamamoto, Kinetic theory with Berry curvature from quantum field theories, Phys. Rev. D 87, 085016 (2013).

[72] C. Manuel and J. M. Torres-Rincon, Kinetic theory of chiral relativistic plasmas and energy density of their gauge collective excitations, Phys. Rev. D 89, 096002 (2014).

[73] J. P. Blaizot and E. Iancu, Lifetime of Quasiparticles in Hot QED Plasmas, Phys. Rev. Lett. 76, 3080 (1996). 